ASSESSMENT OF MORPHOLOGY AND HEMODYNAMICS IN NORMAL AND REMODELED MICROVASCULATURE WITH PARAMETER SENSITIVITY ANALYSIS

\author{
A Thesis \\ Presented to the Faculty of \\ California Polytechnic State University, \\ San Luis Obispo \\ In Partial Fulfillment \\ of the requirements for the Degree \\ Master of Science in Biomedical Engineering
}

by

Shilpi Ghosh

December 2010 
(C) 2010

Shilpi Ghosh

ALL RIGHTS RESERVED 
COMMITTEE MEMBERSHIP

TITLE:

ASSESSMENT OF MORPHOLOGY AND HEMODYNAMICS IN

NORMAL AND REMODELED MICROVASCULATURE WITH

PARAMETER SENSITIVITY ANALYSIS

AUTHOR:

DATE SUBMITTED:

COMMITTEE CHAIR:

COMMITTEE MEMBER: Scott J. Hazelwood, PhD.

COMMITTEE MEMBER: Jesse Maddren, PhD.
Shilpi Ghosh

December, 2010

Trevor R. Cardinal, PhD. 


\begin{abstract}
Assessment of Morphology and Hemodynamics in Normal and Remodeled Microvasculature with Parameter Sensitivity Analysis

\section{Shilpi Ghosh}

The goal of this study was to compare the morphological and hemodynamic

characteristics of normal and remodeled vascular networks in the mouse gracilis muscle. To this end, previously developed models to assess flows and vascular branching were used to assess the normal and remodeled microcirculatory networks. The analysis revealed that the average individual vessel flow rates for vessels of similar caliber and total volumetric flow rates in the networks do not change for vessels of the same caliber after remodeling. Connectivity changes and average diameters primarily change in the larger arterioles after remodeling. A few correlations could be made between architectural and flow properties, however, further modifications in the analysis methods can make future correlations more effective. In order to improve the analysis a parameter sensitivity analysis tool (PSAT) was developed. The PSAT is helpful in teasing apart the individual effects of morphological parameters such as vessel connectivity, vessel diameters, and vessel lengths. In future, another important component that allows the investigator to exclusively alter vessel quantities for all the orders can be added to improve the PSAT. 


\section{Acknowledgements}

I would like to express my enormous gratitude to my thesis advisor, Dr. Trevor R.

Cardinal for his patient guidance, support and advice. Special thanks also to members of my thesis committee, Dr. Scott Hazelwood and Dr. Jesse Maddren for their valuable suggestions and advice for my project. Thanks to Dr. Gabriel Gruionu for providing the images that were used for study in this report. Thanks also goes to Dr. David Clague for his suggestions and assistance with the wall shear stress calculations in this report. My fellow lab mates gave me encouragement during the course of my project.

I am most grateful to my parents and my sister for their constant support throughout the course of my education. My parents have always supported my choices and decisions regarding my education and my career aspirations. My sister has been a source of comfort, humor, and support during periods of stress. Even a light conversation with her on the phone when difficulties arose, gave me the boost of energy that I needed to plough on.

A warm thanks to all my friends from school and college, who have remained in touch with me and given me great joy, encouragement, and love even from far distances.

"Our greatest glory is not in never failing, but in rising up every time we fail." 


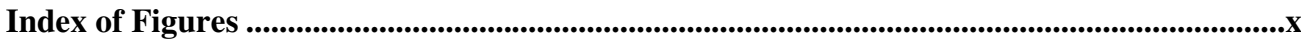

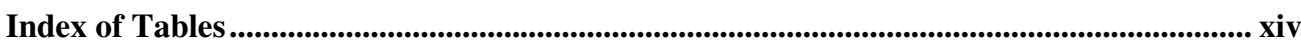

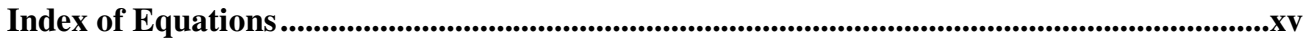

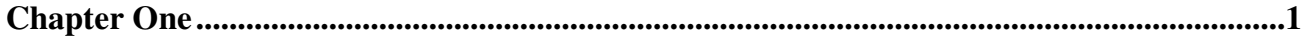

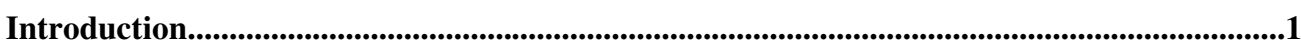

Structure and Function of the Circulatory system........................................................ 1

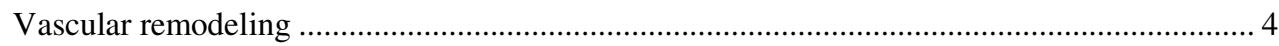

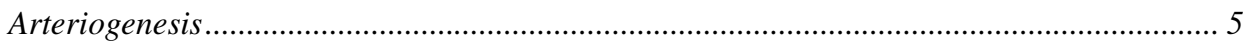

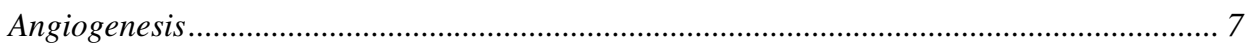

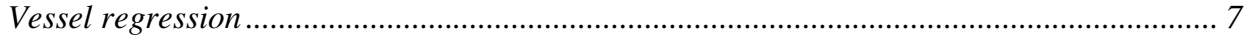

Structural adaptation of the microcirculation during physiological remodeling ................... 8

Structural adaptation of the microcirculation during pathological remodeling.................... 8

Vascular dysfunctions associated with arterial occlusion ................................................. 10

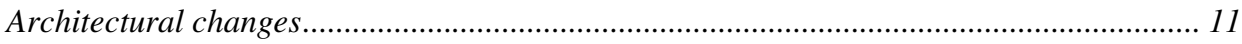

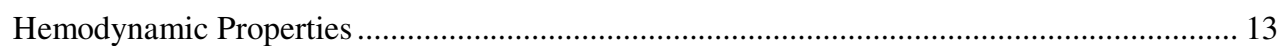

Interrelatedness of hemodynamic, metabolic and morphological parameters ...................... 14

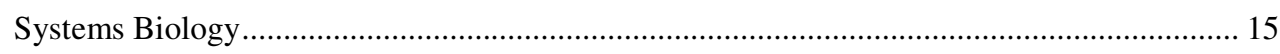

Integration of experimental and theoretical approaches ................................................ 15

Computational approaches used in analyzing vascular network morphology and blood flow 
Numerical approaches used in this report

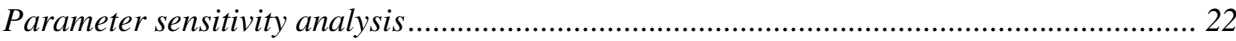

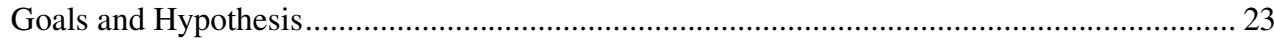

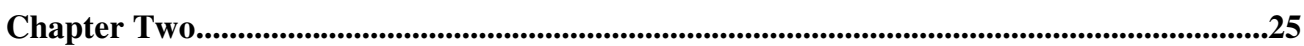

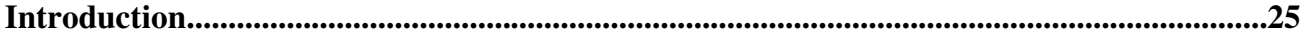

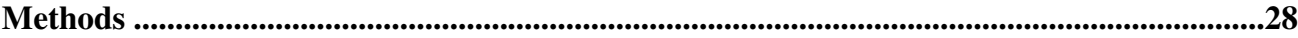

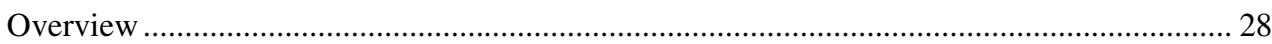

Brief description of vascular anatomy and surgical procedure ......................................... 28

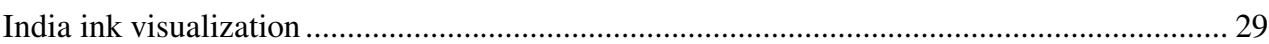

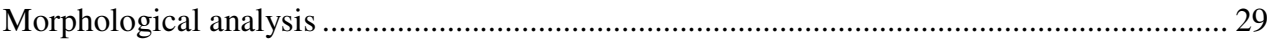

Calculations pertaining to morphological models................................................................ 36

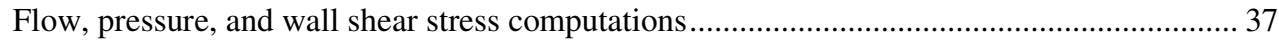

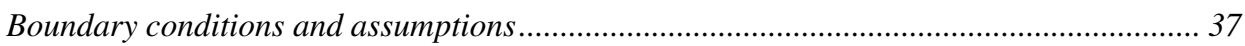

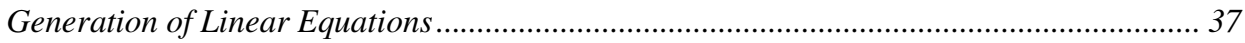

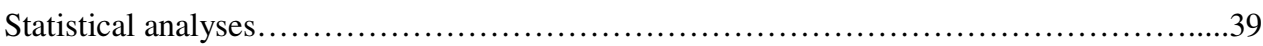

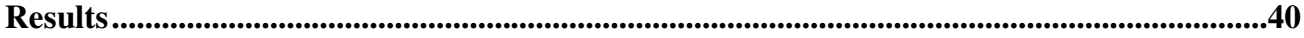

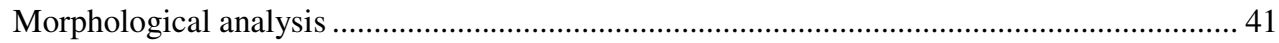

Comparison of network dimensions and topology ..................................................... 41

Comparisons in architecture between normal and remodeled microvasculature.................... 45

Network Connectivity characteristics...................................................................... 45

Comparison of normal and remodeled hemodynamic characteristics .................................. 49

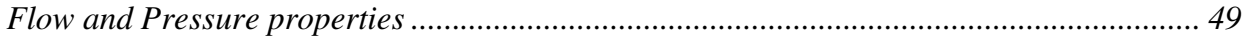




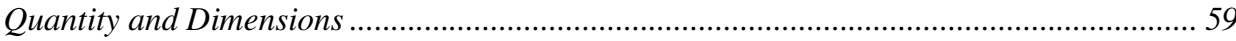

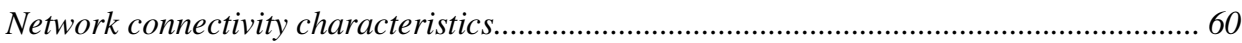

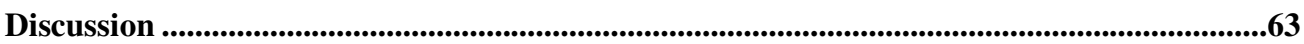

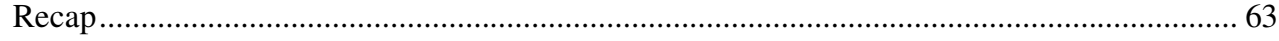

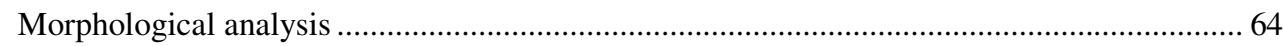

Horton's Law, Strahler ordering scheme, and connectivity matrices ................................... 64

Hemodynamic comparisons between control and remodeled networks ................................ 67

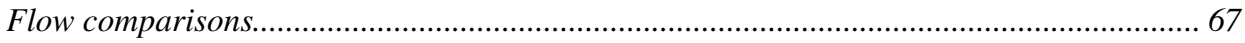

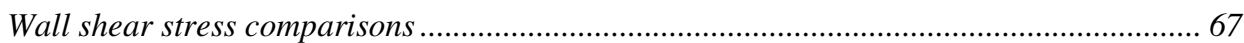

Correlations between architectural changes and hemodynamic alterations post remodeling ... 69

Drawbacks in current models for comparative purposes and future work............................. 70

Vessel ordering and hemodynamic comparisons ........................................................ 70

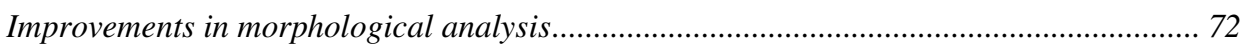

Chapter Three ........................................................................................................................................73

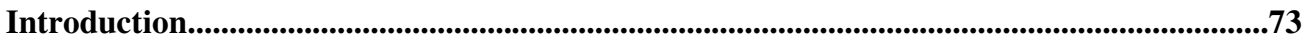

Types of theoretical models in microcirculation research ................................................... 73

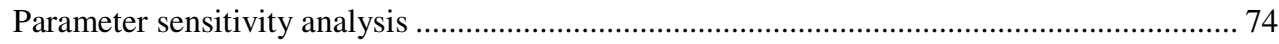

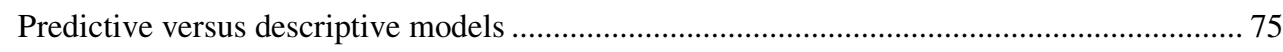

Automation of Strahler ordering and shifting software platforms ..................................... 76

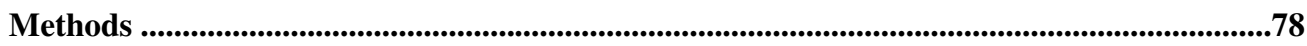

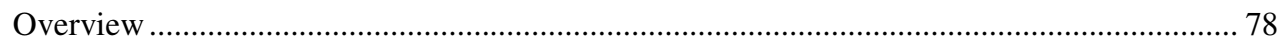




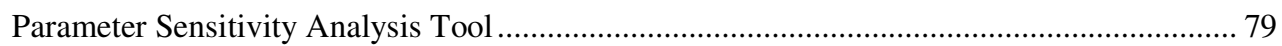

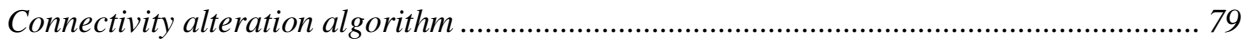

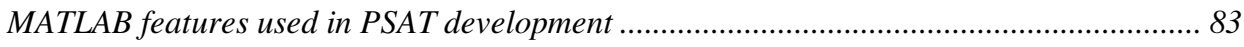

Diameter and length alterations through PSAT .......................................................... 84

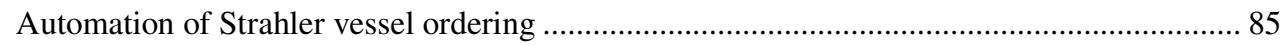

Shifting software platforms from Mathematica to MATLAB ……………………………...... 85

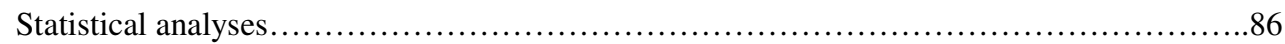

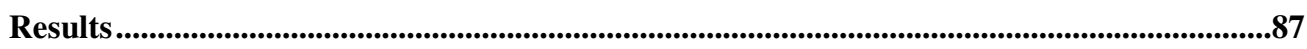

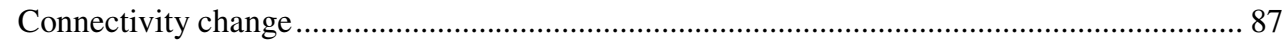

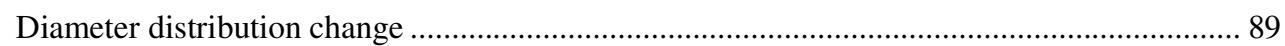

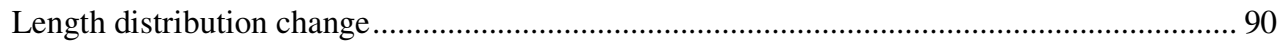

Discussion .....................................................................................................................................................92

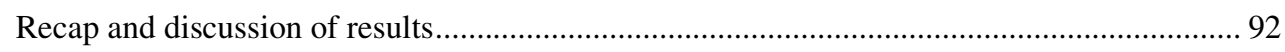

Further improvements in numerical model ......................................................................... 92

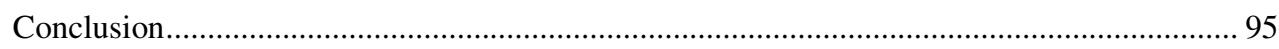

Bibliography ......................................................................................................................................96

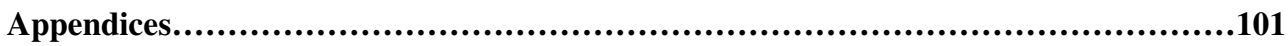




\section{Index of Figures}

Figure 1. Arterial and venous sides of the circulatory system .......................................... 1

Figure 2. Portion of a microvascular network within skeletal muscle tissue.............................. 4

Figure 3. Schematic of ligation and resection of an artery........................................... 6

Figure 4. Normal vascular network in skeletal tissue versus remodeled network 14 days post arterial occlusion 12

Figure 5. Ordering of a branch structure using various ordering schemes. ............................... 18

Figure 6. shows a connectivity matrix representation of an arbitrary branch structure. 21

Figure 7. Schematic representation of imposing diameter bounds from one network to another 27

Figure 8 . Resection of the saphenous artery in the mouse hindlimb 28

Figure 9. Calibration image 30

Figure 10. Three image sections of the ink-stained hindlimb microcirculation stitched together.30

Figure 11. Arteriolar and venular sections of the microcirculation. 31

Figure 12. depicts the smooth bends in the arteriolar trees (traced in red) versus the relative

"jaggedness" of venular trees (traced in blue)

Figure 13. Schematic representation of the arcade structure observed in the mouse gracilis muscle.

Figure 14. Flow direction in the transverse arterioles were determined by the angle with which they separated from the main artery.

Figure 15. depicts Strahler ordering

Figure 16. shows the conversion of a segmental representation of a network to an elemental

one.

Figure 17. shows an arbitrary network branch with labeled nodes and flow directions (in red).. 38 
Figure 18. Normal ink-stained vasculature of mouse gracilis muscle.

Figure 19. Remodeled ink-stained vasculature of mouse gracilis muscle.

Figure 20. Average diameter of vessels across different orders in normal and remodeled microvasculature.

Figure 21. Average quantity of vessels across different orders in normal and remodeled microvasculature

Figure 22. Average length of vessels across different orders in normal and remodeled microvasculature

Figure 23. Total cross sectional area of each order for segments.

Figure 24. Order versus $\log _{10}$ of vessel diameter for normal and remodeled segments. 46

Figure 25. Order versus $\log _{10}$ of vessel length for normal and remodeled segments 46

Figure 26. Order versus $\log _{10}$ of vessel quantity for normal and remodeled segments. 47

Figure 27. Tabular and graphical representation of connectivity of vessel segments in the normal and remodeled vascular networks 48

Figure 28. Average flow rate through individual vessel segments.

Figure 29. Average overall flow rates across the different orders of vessels in the control, remodeled and hypothetical remodeled networks with flow from both ends of arcade (double-sided remodeled) 51

Figure 30. Average pressure in segments of each order.

Figure 31. Comparison of calculated (control and remodeled) and published values of individual segmental flow rates. Calculated values are for the average flow values from the control network data. 
Figure 32. Comparison of overall flow in the control networks with literature. M1, M5, M6, M7, and M8 represent the 5 muscle samples (control only)............................................. 55

Figure 33. Average wall shear stress in individual vessel segments of different orders............ 56

Figure 34. Comparison of calculated wall shear stress (control and remodeled) with literature.. 58

Figure 35. Total cross sectional area of each order for elements............................................ 59

Figure 36. Order versus $\log _{10}$ of vessel diameter for normal and remodeled elements............... 61

Figure 37. Order versus $\log _{10}$ of vessel length for normal and remodeled elements.................. 62

Figure 38. Order versus $\log _{10}$ of vessel quantity for normal and remodeled elements............... 62

Figure 39. Inadequate reflection of branching hierarchy of vessels through diameter based reordering in diameter-defined Strahler method.

Figure 40. Removal of vessel connectivity information from the original network 79

Figure 41. Pictorial representation of connectivity alteration in a remodeled network based on control connectivity ratios for various orders.

Figure 42. A schematic depicting all the different types of bifurcations found post-reordering in the control vascular networks.

Figure 43. Schematic showing the imposition of the diameter distribution in an order of a control network on that of the same order in the remodeled network.

Figure 44. Comparison of average connectivity percentages for control, remodeled, and remodeled networks with altered connectivity.

Figure 45. Comparison of average individual flow rates for all the orders between the remodeled networks and remodeled networks with altered connectivity.

Figure 46. Comparison of average individual flow rates for all the orders between the remodeled networks and remodeled networks with altered diameter distribution 89 
Figure 47. Comparison of average individual flow rates for all the orders between the remodeled networks and remodeled networks with altered length distribution................................ 90

Figure 48. Portion of a tortuous remodeled gracilis artery. ................................................ 93

Figure 49. Velocity field in the model of a normal blood vessel created using COMSOL......... 94 
Index of Tables

Table1. Terms used in morphological analysis............................................................... 31

Table 2. shows an example of data obtained through morphological analysis.......................... 35

Table 3. Number, diameter, and length ratios computed according to Horton's Law................. 45

Table 4. Comparison of the total volumetric flow rate through control, remodeled, and doublesided remodeled networks.

Table 5. Wall shear stress in the control and remodeled vessels for all orders. ......................... 58

Table 6. Number, diameter, and length ratios computed according to Horton's Law.......

Table 7. Comparison of total volumetric flow rates in the altered remodeled network with those of the control and original remodeled networks.

Table 8. Comparison of total volumetric flow rates in the altered remodeled network with those of the control and original remodeled networks.

Table 9. Comparison of total volumetric flow rates in the altered remodeled network with those of the control and original remodeled networks 


\section{Index of Equations}

Equation 1. Wall shear stress equation .......................................................................... 4

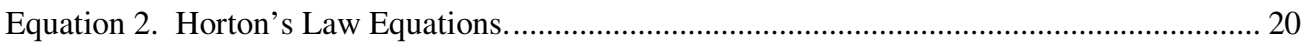

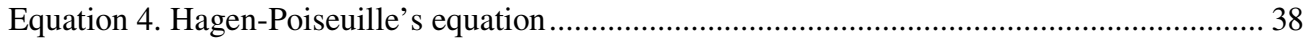

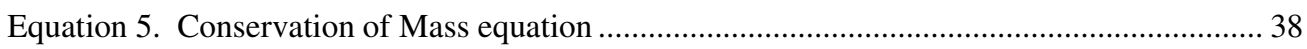

Equation 6. Horton's Law applied to diameter................................................................ 45 


\section{Chapter One \\ Introduction \\ Structure and Function of the Circulatory system}

The circulatory system supplies nutrients to bodily tissues and removes waste products through blood vessels organized in a branching network.

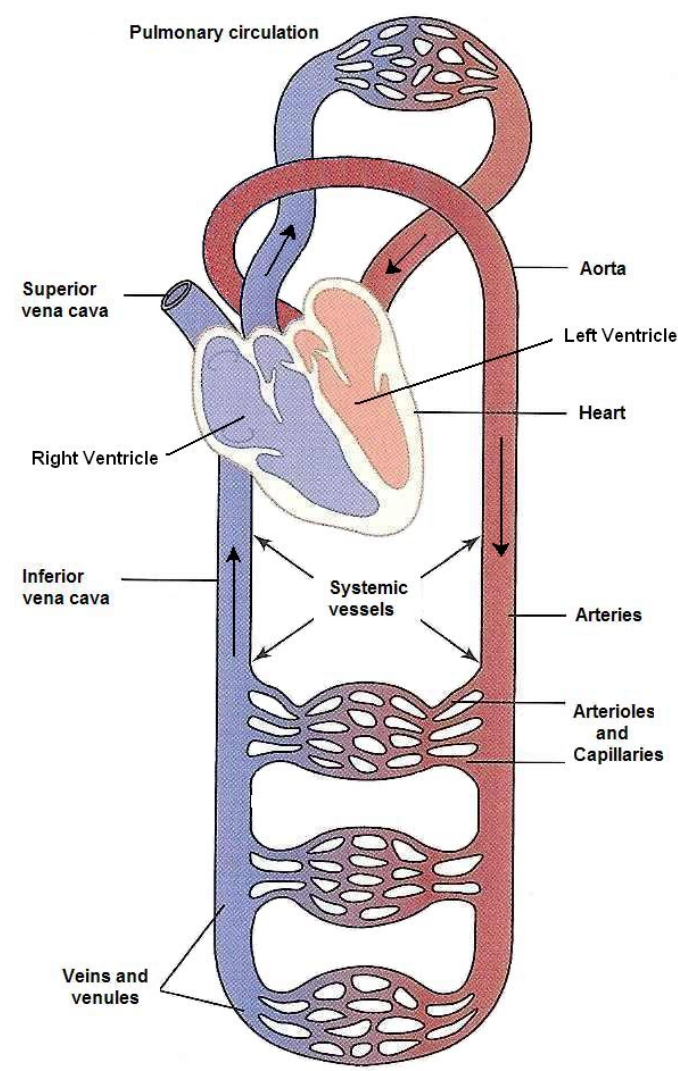

Figure 1. Arterial and venous sides of the circulatory system

The circulation consists of an arterial and a venous side (Figure 1). Arteries transport blood under high pressure and velocity to the tissues, while the veins transport blood away from 
the tissues to the heart at high velocity and low pressure. The left ventricle of the heart pumps oxygenated blood into the aorta which branches into smaller arteries and eventually into tiny capillaries. The capillaries converge to form venules that merge with other venules to form veins. Blood is returned to the heart's right atrium through the inferior and superior vena cava which receives blood from the veins. The deoxygenated blood returned to the heart is then sent to the lungs for oxygenation. The oxygenated blood travels back to the heart and is pumped into the peripheral circulation.

The exchange of nutrients and waste occurs in the microcirculation which consists of blood vessels embedded within a tissue, such as arterioles, capillaries, and venules (Guyton and Hall 2006). Microvascular topology typically comprises of several vascular tree-like branching structures fed by a few supply arteries. Several morphological parameters such as blood vessel length, quantity, diameter, and branching pattern characterize the arteriolar and venular tree structures. The blood vessels of the microcirculation consist of two main cell types, endothelial and smooth muscle cells. Endothelial cells comprise the innermost cell layer of the entire circulation, including the heart, while smooth muscle cells cover the outer surface of arterioles (and arteries) and sparsely populate the surface of venules (and veins). The endothelial cells play a central role in flow control and immune function by sensing hemodynamic, metabolic, and other regulating factors (Ince 2005). The contraction and relaxation of smooth muscle cells that lead to acute changes in blood flow can also be controlled by endothelial cells. Additionally, endothelial cell-to-cell communication transmits information upstream concerning hemodynamic conditions in downstream vessels (Ince 2005).

The microcirculation performs several important functions such as nutrient and waste exchange with the tissue, blood flow control, and trafficking of inflammatory cells and 
cytokines. Capillaries are the sites for nutrient and waste exchange. Demands for oxygen and nutrients are tissue-specific and in most instances each tissue controls its own blood flow. The pressure drop in the circulation occurs primarily in arterioles (within the microcirculation), and therefore this vascular segment is responsible for the control of blood flow. Acute and chronic structural remodeling of blood vessels occurs in response to the changing metabolic needs and hemodynamic conditions within the microcirculation to allow blood flow control. Acute remodeling of blood vessels occurs through vasodilation (increase in the luminal diameter of blood vessels) and vasoconstriction (decrease in the luminal diameter of blood vessels). Vasodilation occurs through relaxation of smooth muscle cells in the blood vessel wall, while vasoconstriction occurs through contraction of smooth muscle cells. Examples of acute blood flow regulation include the control of body temperature (thermoregulation), which is determined by heat loss from the body through blood flow to the skin. Additionally, during exercise muscles require greater blood flow to meet higher energy demands which leads to vasodilation of blood vessels, a condition called exercise hyperemia. Regarding the stimuli that trigger structural adaptation, previous research has shown that vessels respond to hemodynamic forces such as transmural pressure and shear stress at the endothelial surface (Pries and Secomb 2002). The wall shear stress is the force per unit area exerted on the vessel wall due to fluid flow (Equation 1) (Fournier 1998). In response to sustained increases of shear stress, vessels generally exhibit a structural increase of luminal diameter, and at constant volume flow, this reduces shear stress (Pries, Secomb et al. 1998). Vessel networks adapt structurally to maintain a preset relationship between wall shear stress and local transmural pressure (Pries, Secomb et al. 1995). Blood vessels also respond to the metabolic state of the tissue. For example, at a given metabolic demand of the tissue, if flow drops in a portion of the vascular network so that the surrounding 
tissue is poorly supplied with oxygen or other nutrients, then the blood vessels in that portion are stimulated to increase diameter to increase perfusion .

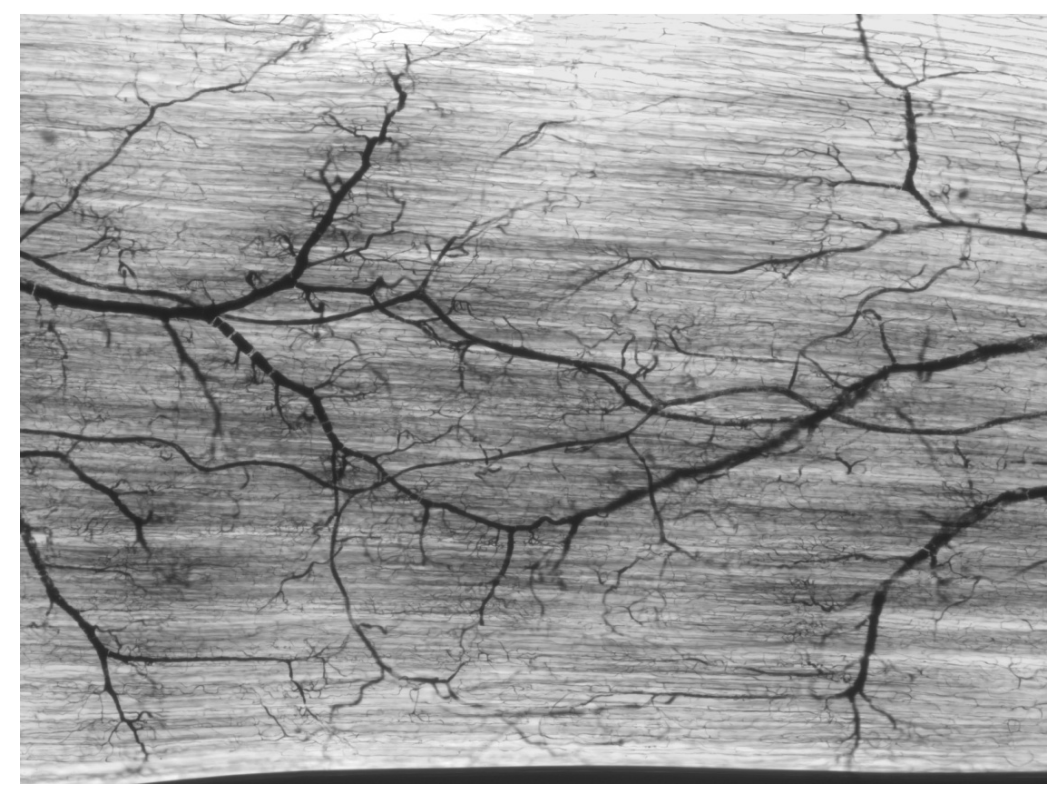

Figure 2. Portion of a microvascular network within skeletal muscle tissue

$$
\tau_{\mathrm{w}}=\left(\frac{4 \mu}{\pi}\right) \frac{\mathrm{Q}}{\mathrm{R}^{3}}
$$

Equation 1. Wall shear stress equation

Relationship between wall shear stress, volumetric flow rate $Q$, viscosity $\mu$ and radius $R$

\section{Vascular remodeling}

As explained previously, acute regulation of flow is mainly accomplished by the contraction and relaxation of smooth muscle cells in vessel walls. However, adaptation of the 
microcirculation to chronic changes in tissue nutrient needs requires more complex remodeling of the vascular network that is triggered by hemodynamic and metabolic stimuli (Pries, Secomb et al. 1998; Pries, Reglin et al. 2001). During chronic adaptation of the microcirculation three types of remodeling processes are observed. The first is arteriogenesis which involves the outward remodeling of collateral arteries and arterioles (bypass routes) adjacent to an occluded artery. The second is angiogenesis which is the formation of new capillaries from pre-existing vessels. The third is vessel regression which leads to a loss of microvessels in the vasculature (Peirce and Skalak 2003).

\section{Arteriogenesis}

The term arteriogenesis, until recently, has been used primarily to describe the process by which existing collateral vessels increase in diameter (Helisch and Schaper 2003). However, the use of this term has now been expanded to include the de novo formation of arterioles from preexisting capillaries (Helisch and Schaper 2003). Both processes of collateral enlargement and arterialization of capillaries contribute to arteriogenesis. Collateral enlargement of preexisting vessels is a predominantly shear stress mediated process. Following the obstruction of flow in an artery, the pressure in the downstream vessels drops substantially. This leads to an elevation in pressure differential across the collateral vessel network and thus, an increase in flow in these vessels (Figure 3). The shear stress experienced by the walls of these vessels is also increased as shear stress is directly proportional to flow.

The increase in shear stress is followed by local inflammation in the vessel wall, monocyte recruitment, remodeling of the extracellular matrix, and stimulation of endothelial cell and smooth muscle cell proliferation (Wahlberg 2003; Heil and Schaper 2004). 
The arterialization of capillaries involves the development of terminal or collateral arteriolar branches from preexisting capillaries through recruitment of perivascular cells that differentiate into smooth muscle cells (Peirce and Skalak 2003). Inflammation and hemodynamic stresses are involved in stimulating the process of arterialization, specifically, circumferential wall stress (stress in the circumferential direction in the wall of the vessel) (Skalak, Price et al. 1998).

A
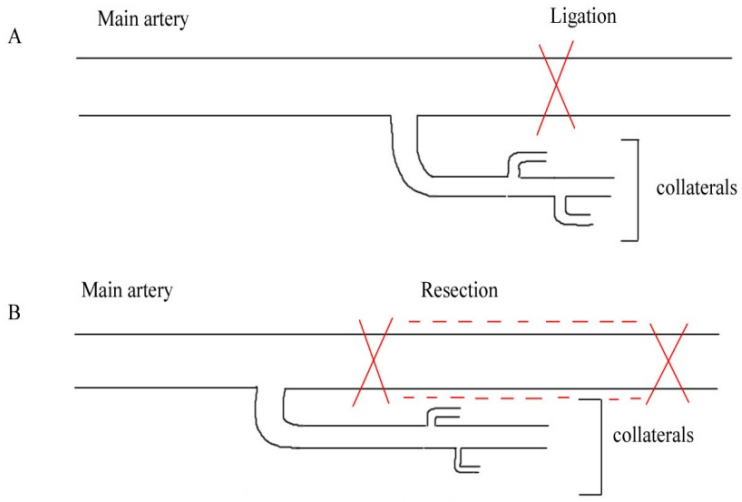

Figure 3. Schematic of ligation and resection of an artery.

A) represents ligation of an artery. Ligation involves tying off the artery at a certain point to prevent flow in the artery beyond the site of ligature. Post-ligation blood is rerouted through collaterals.

B) represents resection of an artery. Resection involves removal of a portion of (or an entire) artery between two points of ligation. Post-resection blood is rerouted through collaterals.

Usually arteriogenesis causes arterioles to become large conductance vessels that maintain blood flow post limb ischemia. The newly enlarged collateral vessels are initially tortuous, but eventually become indistinguishable from a normal artery (Wahlberg 2003). 


\section{Angiogenesis}

Angiogenesis is growth of new capillaries from pre-existing capillaries by the division of vascular endothelial cells. Hypoxia (a decrease in tissue oxygen) is the primary stimulus of angiogenesis (Wahlberg 2003). Hypoxia prevents oxidation dependent degradation of hypoxia inducible factor (HIF) and causes binding of HIF-1 $\alpha$ to the hypoxia response area in the vascular endothelial growth factor (VEGF) gene promoter region. The splicing of the VEGF gene results in five isoforms of VEGF, which play important roles in the angiogenic process (Wahlberg 2003). Next, VEGF mediates an increase in vascular permeability. This occurs through the alteration of cell membrane structure and redistribution of intracellular adhesion molecules. The extravasation of plasma proteins stimulated by VEGF creates an environment to support endothelial cell migration. The degradation of the extracellular matrix is also part of this preparation. Endothelial cells proliferate and migrate through the degraded extracellular matrix and form the neovessel lumen by fusing with the existing vessels. Transforming growth factor- $\beta$ and platelet-derived growth factor stimulate extracellular matrix production and recruit perivascular cells to stabilize the new vessel structures (Wahlberg 2003). Angiogenesis does not contribute significantly to restoration of blood flow, but creates more sites for oxygen and nutrient exchange between the circulation and the tissue.

\section{Vessel regression}

Insufficient perfusion of vessels leads to microvascular rarefaction. Recent studies also suggest that hyperoxia through HIF leads to downregulation of VEGF, which is a survival factor for endothelial cells. Cell-to-cell contact interactions provided by perivascular support cells are needed for vessel maintenance, without which apoptosis of endothelial cells occur. The loss of this cell signal may be involved in vessel pruning (Risau 1997; Peirce and Skalak 2003). A large 
number of small vessels increase the blood flow path length and contribute substantially to flow resistance. Thus, vessel regression helps minimize vascular resistance by pruning downstream vessels.

Structural adaptation of the microcirculation during physiological remodeling

The previously described chronic remodeling processes, namely, arteriogenesis, angiogenesis, and vascular regression often occur under certain physiological states in the tissue, such as during increased physical activity or exercise in skeletal and cardiac muscle tissues. Capillary density measurements show that angiogenesis occurs in normal skeletal or cardiac muscle tissue during increased physical activity. Increased blood flow leading to higher wall shear stress and the greater demand for oxygen are the primary stimulants for capillary proliferation. Capillary sprouting and arterialization in preexisting capillaries with high flow and regression of capillaries with low flow has also been observed in previous research (Hudlicka 1982).

Structural adaptation of the microcirculation during pathological remodeling

Apart from physiological remodeling, chronic structural adaptations in the microcirculation also occur under pathological conditions such as cancer, ischemia, and inflammatory diseases. Angiogenesis is regulated by a balance between pro- and anti- angiogenic molecules in normal physiological states. However, this balance is disrupted in diseases such as cancer which leads to excessive blood vessel growth. The temporal and spatial expression of regulatory molecules of capillary growth is not as well coordinated in tumors as compared to physiological angiogenesis. Tumor vessels lack protective mechanisms that normal vessels possess. For instance, some of them lack functional perivascular cells which protect vessels against changes in oxygen levels or hormonal balance and provide them necessary vasoactive 
control to accommodate metabolic needs. Tumor vessel networks are typically highly disorganized with excessive branching and consequently blood flow is chaotic leading to hypoxic and acidic regions in tumors (Carmeliet and Jain 2000).

In ischemic diseases such as peripheral vascular disease, there is insufficient vascular growth and remodeling after blood flow from a supply artery is reduced or completely obstructed, and blood is rerouted through collateral vessels. In the event that collateral enlargement does not lead to a substantial restoration of flow, chronic ischemia develops. This leads to the starvation of tissue for oxygen and nutrients. Eventually, minor or severe tissue damage can occur depending on the extent of blockage in the main artery. Peripheral artery disease has been reported to affect $7.5 \%$ of the population aged between $60-64$ years in the U.S. In any given year, peripheral artery disease is responsible for 200 lower limb amputations per million in non-diabetic patients and 3900 amputations per million in diabetic patients (Hershey, Baskin et al. 2001). Diabetic patients have been found to have fewer collateral vessels and fewer capillaries which make it difficult for the tissues to recover from arterial occlusion (Wahlberg 2003).

\section{Experimental models of peripheral artery disease}

Often patients with peripheral artery disease have other comorbidities, such as high blood pressure and diabetes that make it difficult to solely study the effects of arterial occlusion on the microcirculation. The body's response to arterial occlusion is affected by factors pertaining to other pathological conditions in the patient's body. Therefore, it has become imperative to model arterial occlusion in animals in order to be able to study the effects of ischemia in isolation.

Animal models of chronic hindlimb ischemia (induced by peripheral arterial ligation) are used to study the effects of arterial occlusion on vascular and parenchymal repair/remodeling. In 
this thesis a mouse model is used to understand the effects of arterial occlusion and ischemia on the microcirculation of skeletal muscle in the hindlimb.

Experimental models of ischemia reveal that the collateral vessels remodel in response to the elevated shear stress experienced by the walls of these vessels. Vessel obstruction causes inflammation which also plays a role in the growth of preexisting collateral arterioles.

Inflammatory cells infiltrate and remodel the vessel wall, and the release of several growth factors stimulates the growth of endothelial and smooth muscle cells. Along with collateral enlargement, hypoxia in the downstream vessels stimulates angiogenesis. After an initial phase of angiogenesis and outward remodeling of existing vessels, vascular regression of capillaries occurs in the second phase and leads to the reduction of vascular resistance (Gruionu, Hoying et al. 2005). Unfortunately, the extensive remodeling observed in experimental models of ischemia is not commonly observed in patients suffering from peripheral vascular disease, perhaps due to the influence of other health complications or aging. Patients typically find it difficult to effectively recover from the ischemic insult through microvascular remodeling that occurs as the body's natural response. Thus, studies such as the one described in this report are important in advancing the understanding of vascular remodeling (through animal models) so that therapeutically induced vascular remodeling can be done more effectively in patients.

\section{Vascular dysfunctions associated with arterial occlusion}

One of the main vascular dysfunctions that arises post arterial occlusion is the reduced responsiveness of the vasculature to vasoactive molecules. Vasoactive molecules are involved in causing the relaxation and constriction of blood vessels. As described previously, vasodilation and vasoconstriction are mechanisms used by small arteries and arterioles for control of blood flow during acute changes in functional demand of tissues. The response of collateral vessels to 
vasoactive molecules such as nitric oxide, acetylcholine and endothelin are shown to be impaired in limb tissues with blocked or reduced blood flow (Takeshita, Isshiki et al. 1998). Impaired vasoreactivity of the vessels post-occlusion is currently being researched to develop a greater understanding of its cause and mechanisms (Kelsall, Brown et al. 2004; Gabhann, Ji et al. 2007).

Apart from impaired vasoreactivity, arterial occlusion also gives rise to architectural changes in the vessel network. Further research is required to establish whether or not changes in architecture in a vessel network are conducive to the restoration of blood flow following arterial occlusion. In previous studies investigators have observed reduced resting blood flow and hyperemia as well as extensive network remodeling of the vasculature following arterial occlusion. The goal of this study is to determine the impact of changes in vascular architecture to alterations in blood flow.

\section{Architectural changes}

As mentioned previously, several morphological parameters such as the resting diameters of blood vessels, blood path length, branching characteristics, and quantity of vessels within a vascular network describe the architecture of the microcirculation. Following arterial occlusion these morphological parameters undergo significant changes that affect the blood flow across the vasculature within the tissue. The diameter of collateral vessels increase in response to the elevation of wall shear stress along the vessels and the blood path length is increased as the blood is re-routed through the collaterals. Additionally, the branching characteristics of the network change due to changes in flow direction and structural remodeling. In the event that angiogenesis and subsequent vessel regression occur as observed in previous research (Gruionu, Hoying et al. 2005), the branching characteristics amongst arterioles and capillaries is affected in a complex manner. 


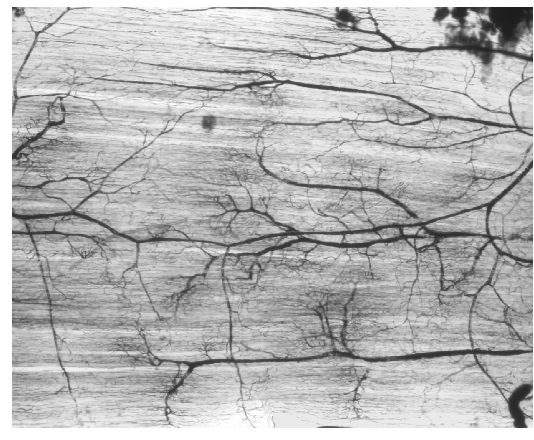

Normal vascular network

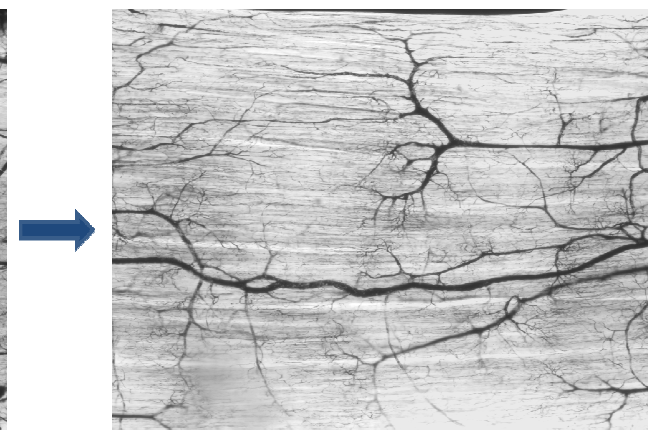

Vascular network 14 days post arterial occlusion

Figure 4. Normal vascular network in skeletal tissue versus remodeled network 14 days post arterial occlusion. The central artery running horizontally across the network in both images is significantly tortuous in the remodeled network compared to the normal one. The quantity of vessels in the remodeled network is also much lower compared to normal.

Understanding these changes can assist researchers and physicians in developing more effective therapies for ischemic diseases. Ischemia, or reduced blood flow, is a problem that occurs in several diseases such as cerebrovascular disease, peripheral artery disease, and coronary artery disease. The restoration of blood flow to a tissue following occlusion is an important but challenging goal as there are several hemodynamic, metabolic as well as cellular and molecular signaling factors involved in the mechanisms of structural remodeling and adaptation in the microcirculation. Some animal studies have reported improved restoration of perfusion as a result of angiogenesis after administration of vascular growth factors or through stem cell therapy (Yang, Deschenes et al. 1996; Ito, Arras et al. 1997; Al-Khaldi, Al-Sabti et al. 2002). Therapeutic angiogenesis has been tried in patients as well. However, the lack of positive trial data has focused researchers and clinicians on the fact that arteriogenesis and angiogenesis are complex processes and simply supplying vascular growth factors to ischemic tissue does not provide effective therapy (Walhberg 2003). It is very important to understand the process and 
effects of revascularization following ischemia in order to gain insight on how vascular growth and remodeling can be used to devise effective therapies.

The architecture and flow across a network changes significantly post remodeling due to considerable changes in the diameters, lengths, and quantities of vessels, which leads to differences in network flow between the normal and remodeled vasculature (Gruionu, Hoying et al. 2005). Along with measuring the morphological characteristics and organizing the structural information regarding the normal and remodeled vascular networks, it is important to estimate the flow rates across the various sections of the network and the total volumetric flow in the network. Architectural changes in the network may suggest an impact on flow. However, only a quantitative assessment of flow in various sections of the network will provide the investigator the means to ascertain correlations between architectural changes and blood flow. In order to calculate the flow rates and pressures in the vessels of the network, it is important to understand the hemodynamic properties of blood.

\section{Hemodynamic Properties}

Blood is a non-Newtonian fluid and its viscosity changes depending upon blood velocity, hematocrit, and diameter of the vessel. The varying viscosity of blood in different vessels affects the shear stress experienced by those vessels, and wall shear stress levels play a very important role in remodeling, as discussed previously. Increased viscosity leads to an increase in flow resistance in the vascular network. Therefore, blood flow rates in the vessels are highly dependent on the fluid properties of blood.

Blood consists of cells suspended in plasma. It also contains a suspension of proteins in varying concentrations. The cellular component of blood consists of erythrocytes (red blood cells), leukocytes (white blood cells) and platelets. The volume of blood occupied by 
erythrocytes is called hematocrit. At low shear rates the red blood cells form aggregates and their characteristic size becomes comparable to the diameter of the blood vessel leading to the nonNewtonian properties of blood. At high shear rates the red blood cell aggregates are broken apart and the blood behaves closer to Newtonian.

Hematocrit, and thus blood viscosity, also varies with vessel diameter - small diameter vessels have lower hematocrit levels compared to large diameter vessels and hence, lower viscosity. This reduction in the blood viscosity due to vessel diameter is referred as the FahraeusLindquist effect (Fournier 1998).

\section{Interrelatedness of hemodynamic, metabolic and morphological parameters}

As described in the previous discussion on structural adaptation of blood vessel networks in normal and pathological conditions, there are several interrelations between the hemodynamic, metabolic, and morphological parameters that characterize the microcirculation. For example, wall shear stress and tissue oxygen levels affect vessel diameter. Vessel diameter affects blood flow rate which in turn affects wall shear stress and blood viscosity. Also, blood viscosity alterations affect vascular conductance and thus, blood flow rate.

When several factors interact and affect each other through complex mechanisms to produce a resultant phenomenon, it becomes challenging to understand how the perturbation of one factor or a combination of more than one such factor will alter the observable resultant phenomenon. However, prior to attempting to tease out the individual roles of each factor, it is useful to create a computational model of the event that can afford the investigator additional modes of insight through theoretical experimentation. It helps greatly in such situations to elucidate as much as possible the underlying mechanisms of such complex phenomena based on experimental results. Thereafter, hypotheses can be developed regarding the rules of interaction 
between the various constituent factors to create a theoretical model.

The results of in silico experiments run on these theoretical models can be compared to results of similar experiments run in vitro or in vivo. Based on the comparisons of the predicted and experimental results, new insights can be gained on the mechanisms and alterations can be made to existing hypotheses if needed to develop further understanding. It is also possible to gain insight on a process by creating a descriptive model that attempts to organize a large amount of data pertaining to a system and then reveal mathematical relations between existing parameters, which are otherwise not obvious. These mathematical relations are not based on underlying mechanisms of the biological process. Nevertheless, they may reveal interesting patterns in the data which can be altered in silico or experimentally to study how changes to such observable patterns are related to interactions between the different parameters. Such an integrative approach leads to clearer and faster development of knowledge regarding processes on a systems level.

\section{Systems Biology}

\section{Integration of experimental and theoretical approaches}

Developing a system-level understanding of a biological process requires an integrative use of the knowledge and tools available through computer science, chemistry, physics, mathematics, biology, statistics and other disciplines to elucidate the underlying mechanisms of a system level phenomenon. The approach involves discovering how individual parameters are interconnected and how their interactions manifest as observable patterns on a systems level.

Experimental and computational approaches have been used previously in microcirculation research. Heterogeneities in hemodynamic and geometric parameters of the rat mesentery microvascular networks and their correlations have been studied using experimental 
data and theoretical models (Pries, Secomb et al. 1995). Model predictions of diameter variations after remodeling in the mouse hindlimb have been compared against experimental results to test hypotheses regarding remodeling stimuli (Gruionu, Hoying et al. 2005).

Computational approaches used in analyzing vascular network morphology and blood flow

Computational models have been used previously to study the architecture of the microcirculation in various tissues such as the pig heart, cat mesentery and the rabbit omentum and relate geometrical features to blood flow through the vascular networks (Zweifach and Lipowsky 1977; Jiang, Kassab et al. 1994; Kassab, Berkley et al. 1997; Kassab 2000). Ordering schemes were used to section and organize portions of the microcirculation based on vessel diameter and branching hierarchy in these studies. Additionally, simplifications and assumptions regarding hemodynamic properties were used to estimate blood flow rates in the networks. The network-based analysis conducted in such research involves calculating flow across all the different vessels and organizing information on flow rates and pressure differentials across individual vessels. This approach has been very useful in revealing the heterogeneities that exist in the values of these parameters throughout the vessel network. From these studies it has become clear that a "typical vessel" approach that averages the properties of vessels, such as the Krogh cylinder model that averages the properties of capillaries to estimate perfusion in a section of tissue, does not reveal flow and pressure variations throughout vessels of a similar morphology. There is variation in the values of hemodynamic parameters, such as pressure gradients and shear stresses, even for vessels such as capillaries that belong to the most topologically homogeneous category (Pries, Secomb et al. 1995).

The analysis of the tree-like arteriolar and venular networks in the microcirculation involving treatment of the vessels as individual units of a network with unique geometric and 
hemodynamic properties requires a method of organizing the data on a large number of vessels. Averaging the properties of vessels as is done for the Krogh cylinder model, leads to a loss of detail concerning the variations in structure and hemodynamics of vessels of similar topology.

Over the years, approaches similar to those used in the study of branching trees formed by rivers have been very useful in studies on the respiratory and microcirculatory systems. The mathematical properties of branching trees as revealed by these models of analysis have been useful in creating a basis of calculating physiological variables such as shear rates and intravascular pressure. Without a method for organizing the geometric information pertaining to these networks, such calculations would become a very tedious task both to execute as well as to present. Another benefit of these models is that they assist researchers in determining underlying design principles of these trees by providing clues as to what factors influence network topology and dimensions (Horsfield 1976). Additionally, morphological variations in the networks due to disease can be studied for particular types of tissue affected by the pathological conditions.

The primary types of ordering systems are Horsfield, Strahler, and generational schemes. Horsfield and Strahler ordering systems assign the lowest order to the furthermost (terminal) vessels in a branching tree-like structure, and order levels increase as vessels converge along the branching hierarchy (Horsfield 1976). In generational ordering schemes the terminal vessels of the tree-like structure are assigned the highest orders and order levels decrease as vessels diverge along the branching hierarchy (Wiedeman 1968). The figure below shows an example of ordering networks based on the Horsfield, Strahler, and generational methods 


\section{(Figure 5):}

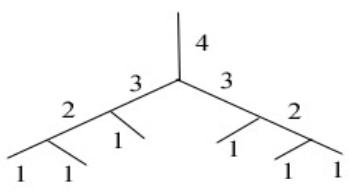

a

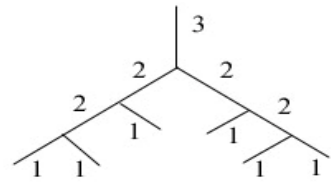

b

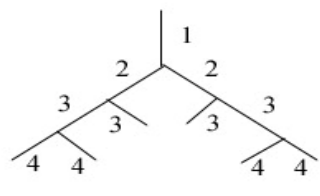

c

Figure 5. Ordering of a branch structure using various ordering schemes. a) depicts ordering of a branch structure based on Horsfield ordering scheme. b) depicts ordering of a branch structure based on Strahler ordering scheme. c) depicts ordering of a branch structure based on Generational ordering scheme

The use of Horsfield ordering leads to a greater number of orders compared to Strahler ordering in asymmetric trees (the term "asymmetric" is used with respect to the number of branching points and not dimensions or branching angles). The highest order obtained for a tree using Horsfield ordering does not provide an accurate representation of the level of branching present in the tree, since the addition of every branch segment leads to an increase in the order number of the parent segment irrespective of the values of the daughter orders. Strahler ordering, on the other hand, takes into account the order of the daughter branch segments while assigning parent orders. Using the Strahler method, the orders of branch segments very close to terminal branch segments are not allowed to increase rapidly. Terminal branch segments are usually found at the very bottom of a branching hierarchy, and vessels connected to them are likely to possess structural properties (such as diameter in the case of blood vessel segments) similar enough to justify being grouped together within the same order. Thus, the branching pattern of the tree structure is afforded a better representation by the Strahler method. In the generational ordering scheme, the orders of terminal branch segments indicate the degree of branching that is 
present in their respective parent branch structure. The order number of any branch segment indicates its branching distance from the parent segment. However, the terminal segments are not grouped under the same order and thus, the convenience of performing statistical analysis on their structural and hemodynamic properties (such as length, quantity, or flow rates) is no longer available.

\section{Numerical approaches used in this report}

Horton's Law of stream numbers was initially developed to describe the branching patterns of streams and riverbeds. This approach has been modified since then and used in studying the microcirculation as well. Several microvascular networks have exhibited branching characteristics that are consistent with this law (Fenton, Zweifach 1981; Jiang, Kassab 1994; Kassab, Berkeley 1997; Kassab 2000). Horton's Law states that certain morphological parameters belonging to an order (a scheme used to describe branching level) will form an inverse geometric sequence with order number. This is to say that a value can be multiplied by a constant to reach the next value in the sequence. According to Horton's Law, quantity, diameter, and length form inverse geometric sequences with order (Fenton and Zweifach 1981). A network consistent with Horton's Law can be described using the equations listed under (Equation 2)

(Kassab 2000). 


$$
\begin{aligned}
& \log _{10} D_{n}=a+b n \\
& \log _{10} N_{n}=a+b n \\
& \log _{10} L_{n}=a+b n
\end{aligned}
$$

Equation 2. Horton's Law Equations.

\section{$D_{n}, N_{n}$, and $L_{n}$ represent the average diameter, quantity, and average length of vessels of order $n$,} respectively.

While Horton's Law reveals an interesting property in branching systems, it is limited to highlighting patterns between adjacent orders. Therefore, modifications were made to this approach to create the Strahler ordering system that captured the branching characteristics of the entire network based on connectivity of vessels (Jiang, Kassab et al. 1994). In the Strahler system, every vessel between two nodes (branching points in the network) is considered a segment and possesses an order number based on its connectivity to other vessels in the network. The terminal branch vessels in the network are assigned order 1 . When two order 1 vessels meet the confluent vessel is called an order 2 and so on. When two vessels of different orders come together, the confluent vessel retains the order of the higher order vessel. For example, when an order 1 and an order 2 vessel come together, the confluent vessel is assigned an order 2 . Further modifications made on the Strahler system resulted in the diameter-defined Strahler system that established ordering of vessel segments based on connectivity as well as vessel diameter. In the Strahler system of ordering if two vessels of order 2 came together and the confluent vessel was not larger than either of the two vessels in diameter, the vessel would be called an order 3 , whereas the diameter-defined method would assign the confluent vessel an order 2 . The diameter-defined Strahler ordering system avoids the overlap of vessel diameters between vessels of different orders. In the new diameter-defined Strahler method, statistical analysis is 
used to define orders for vessels based on connectivity, average diameter within orders, and diameter standard deviations. This method is described in greater detail later in the report.

A second modification incorporated into the Strahler system was the creation of elements from identical segments connected in series. An element consists of same order segments connected in series with each other. This led to a more accurate representation of the architecture of the network, especially with respect to the lengths of vessels in different orders. The creation of an elemental representation of a network from a segmental one is described in detail later in this report.

Finally, a third feature incorporated into the analysis along with Horton's law and the diameter-defined Strahler ordering system is the connectivity matrix. The connectivity matrix is very useful in depicting the branching characteristics of vessels of different orders within the network. For example, vessels of order $n$ may branch from vessels of order $n+1, n+2, n+3$ and so on. The number of such branches occurring in a network for each type of order shows the distribution of vessels arising from particular parent vessel orders. A matrix representation of such connectivity characteristics is very useful in assessing changes in overall branching characteristics between two or more networks. An example of a connectivity matrix is shown below (Figure 6):

\begin{tabular}{r|r|r|r|r|}
\hline & 1 & 2 & 3 & 4 \\
\hline 1 & 0 & 6 & 0 & 0 \\
\hline 2 & 0 & 2 & 2 & 0 \\
\hline 3 & 0 & 0 & 0 & 0 \\
\hline 4 & 0 & 0 & 0 & 0 \\
\hline
\end{tabular}

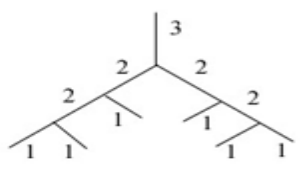

Figure 6. shows a connectivity matrix representation of an arbitrary branch structure 
All of the previously described components of the model used to assess the morphology of vascular networks in this report are useful in finding patterns of regularity and order in seemingly chaotic network structures. For instance, Horton's Law reveals whether there is a pattern in the change of diameter, length, or quantity of vessels from one order to the next. Also, the diameter-defined Strahler ordering system affords the unique capability of capturing the connectivity of vessels in the network as a whole and further grouping them such that there is no overlap of diameters between different orders. Using this ordering system, segments of the network are portioned into groups with distinct diameter and connectivity characteristics and the investigator can compare the variation of morphological and hemodynamic parameters across these different orders. In addition to helping assess different portions of the same network, Horton's Law and the diameter-defined Strahler ordering system allow the investigator to compare topological differences between two or more networks. For instance, it is interesting to study whether or not there are any patterns of regularity in the architecture of a vascular network before remodeling due to a pathological condition. Additionally, if any patterns are discovered, further studies can be performed to determine whether the pattern persists or changes in the remodeled network. This information can be highly useful in improving tissue function and patient health in the future, if it becomes therapeutically possible to control network structure. In this report, the topological differences between vascular networks before and after remodeling due to an arterial occlusion have been compared.

\section{Parameter sensitivity analysis}

As described previously, the morphological characteristics that describe a vascular network include vessel diameters, lengths, and the connectivity between vessels. Once this information is obtained from a network and flows and pressures are calculated, the influence of 
perturbing these parameter values on flow rates and pressures across the network can be studied. While comparing two vessel networks, for instance, one before and the other after remodeling subsequent to the occlusion of an artery, the ability to impose the morphological properties of the normal network on the remodeled to observe how the changes in each parameter affect network hemodynamics becomes very useful in studying the effects of remodeling. For example, if diameters and lengths are different, parameter sensitivity analysis allows the investigator to determine the relative contribution of each morphological change on any observed differences in blood flow.

The development of a parameter sensitivity analysis which allows the researcher to alter morphological parameters such as vessel connectivity, diameter distributions, and length distributions of vessels across orders is discussed in subsequent sections.

\section{Goals and Hypothesis}

This project has three main objectives. The first objective was to assess the differences in network morphology and flow characteristics between normal and remodeled vascular networks using Horton's law, the diameter-defined Strahler system, and the connectivity matrices. The hypothesis is that Horton's law, diameter-defined Strahler system, and connectivity matrices can sufficiently characterize the normal and remodeled vasculature for comparative analysis of morphological and hemodynamic characteristics.

The second objective was to develop the parameter sensitivity analysis tool. The tool will allow the researcher to arbitrarily alter the vessel connectivity, diameter distribution, and length distribution of a given vascular network. Using this tool the researcher can then quantitatively determine the individual influence of each parameter alteration on flow through the network. 
The third goal of the project was to automate the process of vessel ordering and make flow calculations more user-friendly by shifting software platforms from Mathematica to Matlab. The computational processes involved in morphological analysis and flow calculations can be made more user-friendly and less time consuming this way. 


\section{Chapter Two \\ Introduction}

Following arterial occlusion and during ischemic revascularization, blood vessels respond by sprouting new capillaries (angiogenesis) and enlarging and remodeling pre-existing arterioles (arteriogenesis). Abnormal vascular remodeling subsequent to arterial occlusion can contribute to a number of cardiovascular diseases such as hypertension, atherosclerosis, and peripheral artery disease (Contreras, Robles et al. 2006). Studying vascular remodeling post-arterial occlusion and understanding the mechanisms underlying the revascularization process can contribute towards the design of therapies for several life-threatening diseases in which vascular growth and remodeling is impaired (or something along those lines).

Comparatively, arteriogenesis plays a greater role than angiogenesis in the restoration of blood flow (Unthank, Nixon et al. 1995; Hershey, Baskin et al. 2001; Scholz, Ziegelhoeffer et al. 2002; Contreras, Robles et al. 2006). Chronic occlusion of a feed artery in skeletal muscle produces several biochemical and mechanical stimuli. Angiogenesis has been found to be influenced mainly by the biochemical factors, whereas the mechanical factors such as shear stress and circumferential wall stress are primarily known to determine the adaptation of collaterals.

Most of the theoretical studies performed on vascular remodeling involve network analysis of flow and pressure in response to metabolic and hemodynamic stimuli. The predictions of these computational models of adaptations are then compared with experimental observations to better understand the mechanisms underlying microvascular adaptation (Pries, Secomb et al. 1998; Pries, Reglin et al. 2003; Gruionu, Hoying et al. 2005; Gabhann, Ji et al. 2007). In this report we focus on descriptive comparisons of the vascular remodeling phenomenon rather than the underlying mechanisms. Descriptive models can give insights into 
the remodeling phenomenon that can aid in formulating better hypotheses for experimental studies.

Descriptive models of the architecture of vascular networks can be used to solely assess the changes that occur in the topology and dimensions of normal networks during the remodeling process. On one hand, a mechanistic understanding of remodeling gives us insight into how the system changes in response to hemodynamic and biochemical stimuli. On the other hand, quantitative analysis of the geometrical changes that occur in the network can give us insight into the type and extent of the changes that occur in the system. For example, patterns of remodeling are different in cases of tumor angiogenesis, post acute infarction, and skeletal or cardiac muscle hypertrophy. In the former two cases it seems that vessels grow from random points on preexisting vessels toward a center of pathology, which may be the site of angiogenic factor production. In the latter cases the adaptation serves to enlarge the existing network without altering the fundamental branching pattern (Skalak and Price 1996). The current study aims to discover whether ischemia will lead to any changes in the general network topology.

In attempting to restore flow through an insufficiently perfused remodeled vasculature it is important to know both how to induce changes in the network, have a good estimation of the type of morphological changes needed to restore flow, as well the usual tendency for remodeling vasculature in an ischemic environment. For example, during the remodeling process there may be portions of the vasculature where flow has been restored to values near or beyond normal. In such cases identifying points in the network from which new branches can lead to better perfusion need to be determined, so that the restoration of flow achieved by remodeling is not compromised during therapy, and the induced remodeling targets the insufficiently perfused regions of the vasculature as much as possible. For example, if it is the arterialization of 
capillaries that is needed, then appropriate stimuli for the same can be applied if possible; if angiogenesis can restore flow back to normal without greatly increasing peripheral resistance, then appropriate dosage of angiogenic stimuli can be applied.

The following section of this paper reports the changes observed in the morphology of the normal mouse gracilis muscle microcirculation and the resultant hemodynamics after remodeling subsequent to resection of a supply artery. Various anatomical features such as the alterations in diameter distributions and branching across the different orders of vessels have been assessed using previous models. This assessment can be used to hypothesize which morphological characteristics need to be changed in order to restore flow through the remodeled network. It can also be used to determine the quantitative impact of altering particular network characteristics on network flow properties.

In making comparisons of flow across the vasculature between the normal and remodeled networks, it was decided that the diameter bounds of the orders in the normal vessel network would be imposed on the remodeled. This was done to ensure that the flow comparisons were being made between vessels of similar caliber, and since vessel diameter most significantly affects flow, according to Hagen-Poiseuille's equation, comparisons were made between vessels with diameters that fell within same upper and lower bound diameter ranges (Figure 7).

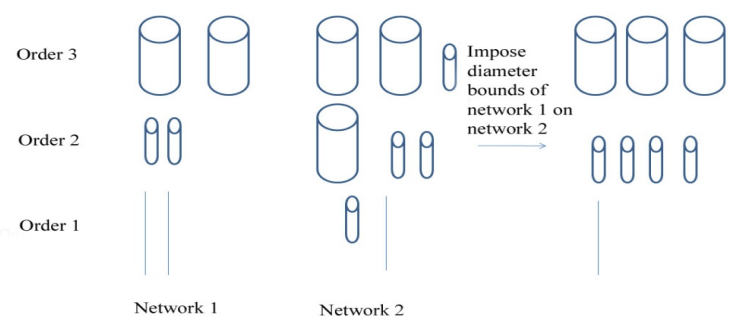

Figure 7. Schematic representation of imposing diameter bounds from one network to another 


\section{Methods \\ Overview}

In this chapter the details of the methods for staining, imaging, performing morphological analysis, and computing blood flow values are covered.

The photomicrographs used in this report for the purposes of morphological analysis were generated during an experiment performed previously (Gruionu, Hoying et. al 2005). A brief summary of the methods applied to generate these images will be presented. More details on the methods can be accessed in the report on the previous experiment.

\section{Brief description of vascular anatomy and surgical procedure}

The gracilis artery (GA) is the main blood supply to the gracilis anterior muscle. The artery is fed by two other arteries, the muscular branch of the femoral artery and the saphenous artery. The GA runs parallel to the muscle fibers over almost the entire length of the muscle and transverse arterioles (TAs) branch off the GA intermittently and supply the capillaries.

The blood supply to the GA from the saphenous side was disrupted by removal of the saphenous artery between the two ligature points indicated in (Figure 8):

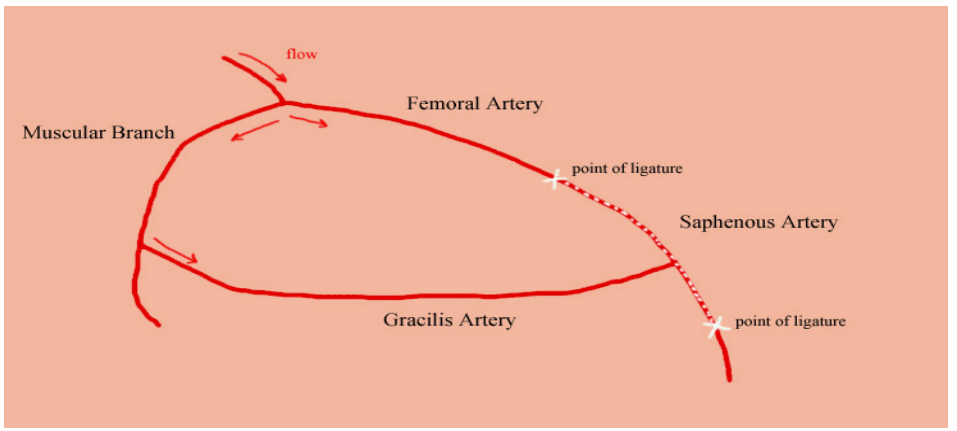

Figure 8. Resection of the saphenous artery in the mouse hindlimb 
Both the saphenous artery and vein were removed. The mice were euthanized

$1,2,7,14,21,28$ or 56 days after surgery. The untreated control group mice received no surgery. The saphenous artery of the sham control mice was exposed but maintained intact. This sham surgery is performed to ensure that the control system receives the same effects due to exposure of the tissue and microcirculation as does the remodeled. The differences between the control and the remodeled would then be only due to the effects of the resection surgery on the remodeled (Gruionu, Hoying et. al 2005).

\section{India ink visualization}

The left ventricle was cannulated with a PE60 catheter and the blood was flushed with PBS containing heparin and sodium nitroprusside. Ink solution was immediately perfused thereafter into the vasculature at a constant pressure of $90-100 \mathrm{mmHg}$. The ink was then allowed to stabilize in the vessels. The gracilis muscle on both sides was dissected, placed on a microscopic slide to prevent it from curling and was subsequently dehydrated in a graded series of alcohol solutions $(25,50,75,95$ and $100 \%$ ethanol) for $\sim 12$ hours in each alcohol dilution. Finally, the muscles were cleared in $100 \%$ methyl salicylate. The vasculature was analyzed with a stereomicroscope (Gruionu, Hoying et. al 2005).

\section{Morphological analysis}

The morphological characteristics of the vascular network were measured from the images of the normal and remodeled vasculature using Image $\mathbf{J}$ software and Adobe Photoshop. The following image was used for the calibration of all the images (Figure 9): 


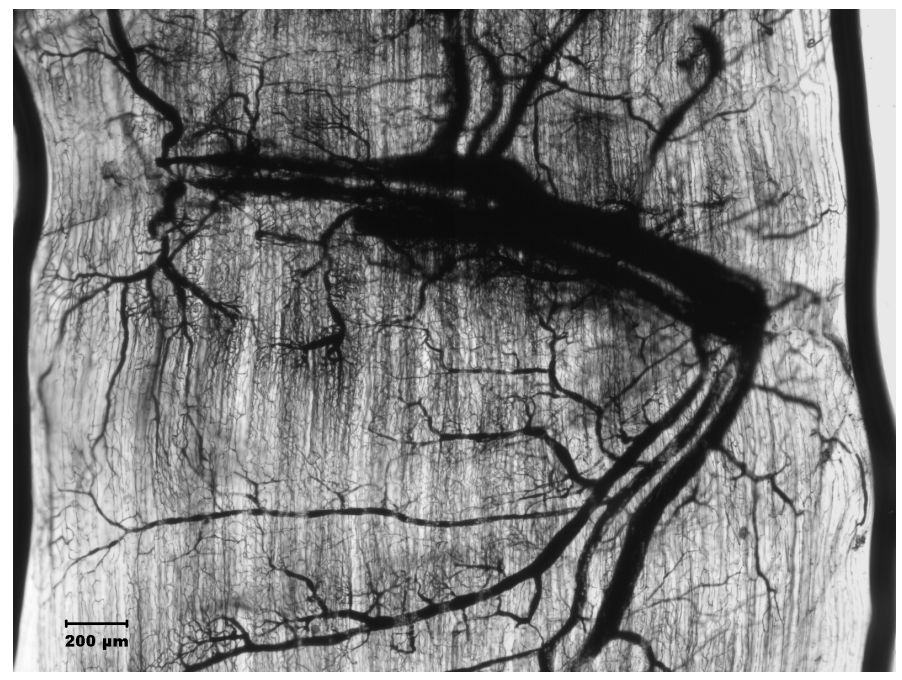

Figure 9. Calibration image

The images of the microvasculature for each tissue sample were obtained in three

sections. The sections were then aligned and stitched together in Adobe Photoshop as shown in

(Figure 10).

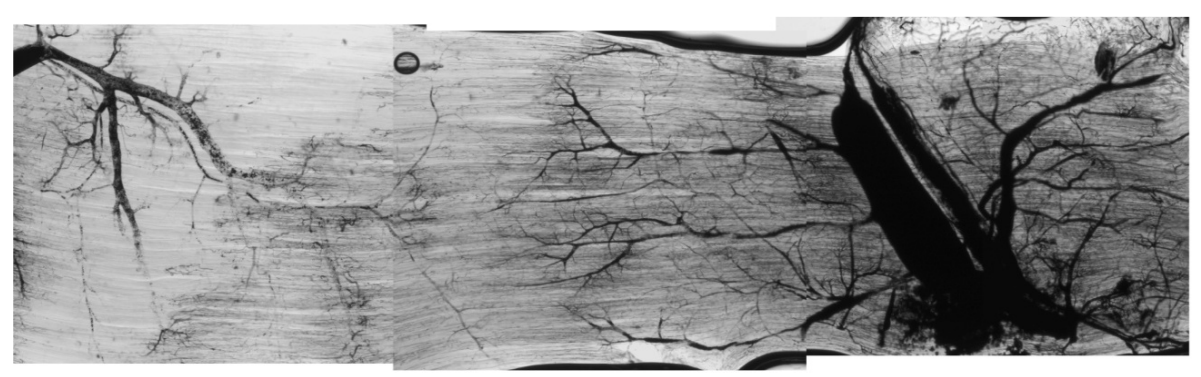

Figure 10. Three image sections of the ink-stained hindlimb microcirculation stitched together.

Table 1 defines the various terms used in morphological analysis. 
Table1. Terms used in morphological analysis

\begin{tabular}{ll}
\hline Term & Definition \\
\hline Vessel segment - & Vessel between two branching points in the network \\
Node - & Branching point in the network \\
Split point - & Point where an arcade is split in half \\
Label - & Unique ID number for each vessel segment in the network \\
Order - & Classification of vessel based on location in the branching \\
& hierarchy \\
Parent - & Feeding vessel upstream of a segment \\
Element - & Multiple segments of identical orders connected in series
\end{tabular}

The part of the microvasculature depicted in red in the following figure depicts the portion of the network aimed for analysis (Figure 11):

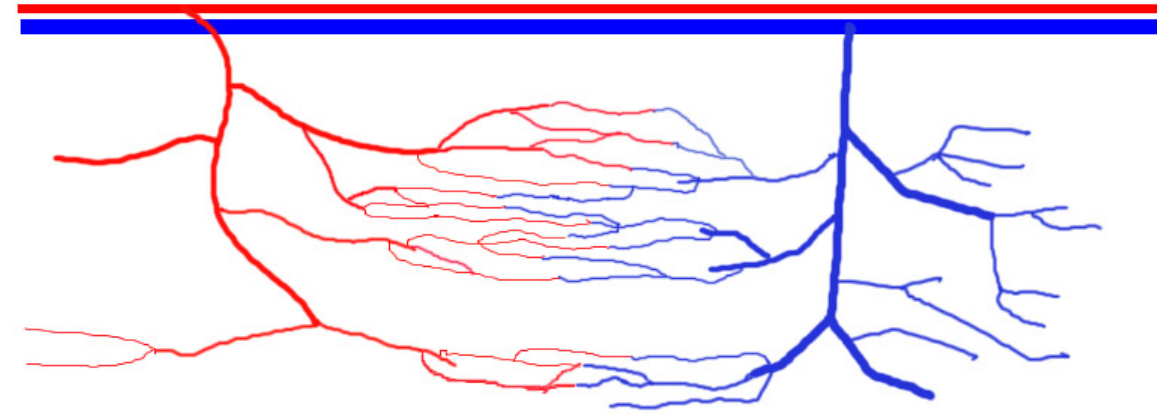

Figure 11. Arteriolar and venular sections of the microcirculation. The red portion of the vascular network depicts the arteriolar part of the microcirculation and the blue portion depicts the venular part.

Two important principles observed in the circulation were used to identify the main arteries and veins and to trace the flow path running from the main artery through the arterioles to the capillaries in the vasculature. The first being that arteries typically run parallel to veins and the second being that arteries usually have smaller diameters compared to the veins running alongside them. While performing the morphological analysis another pattern pertaining to the 
structure of arteriolar and venular vessels was observed in the vasculature. Arterioles typically follow smooth bends along the plane of the muscle, while venular branches exhibit a relative "jaggedness" in their structure that makes them distinct from the arteriolar trees. The following figure illustrates this feature (Figure 12):

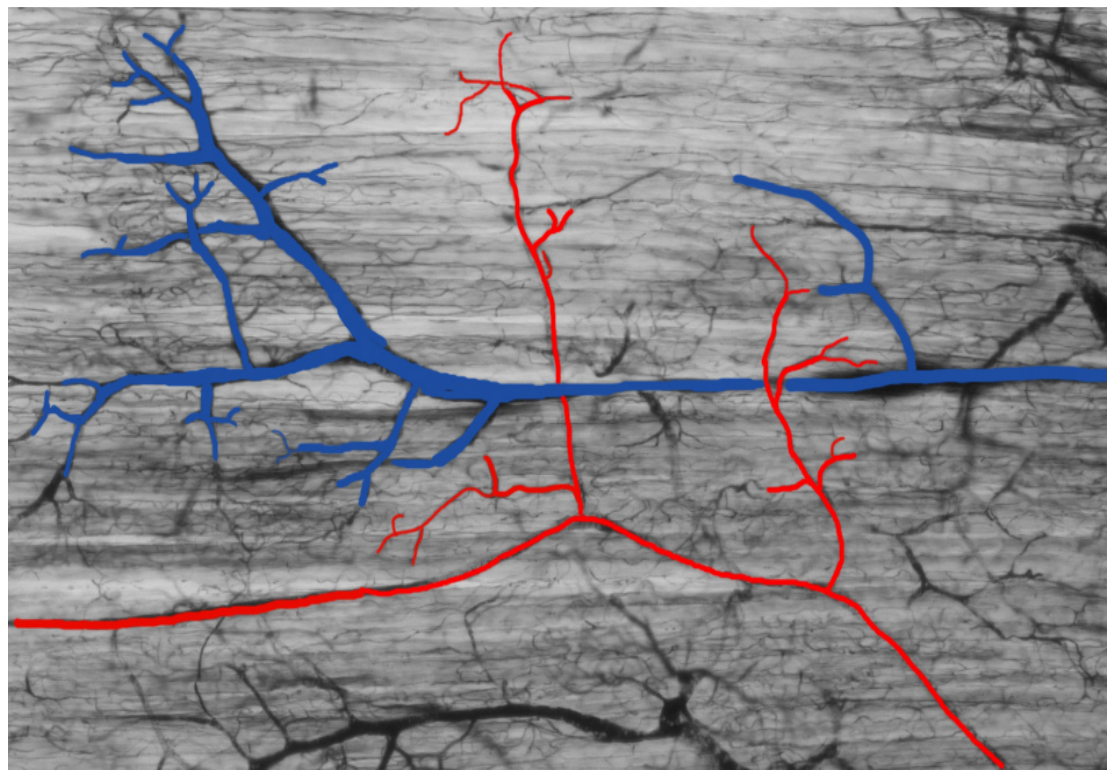

Figure 12. depicts the smooth bends in the arteriolar trees (traced in red) versus the relative "jaggedness" of venular trees (traced in blue)

The gracilis artery traverses the entire length of the muscle and is fed by both the muscular and saphenous branches at its two ends, and forms an arcade structure (Figure 13). 


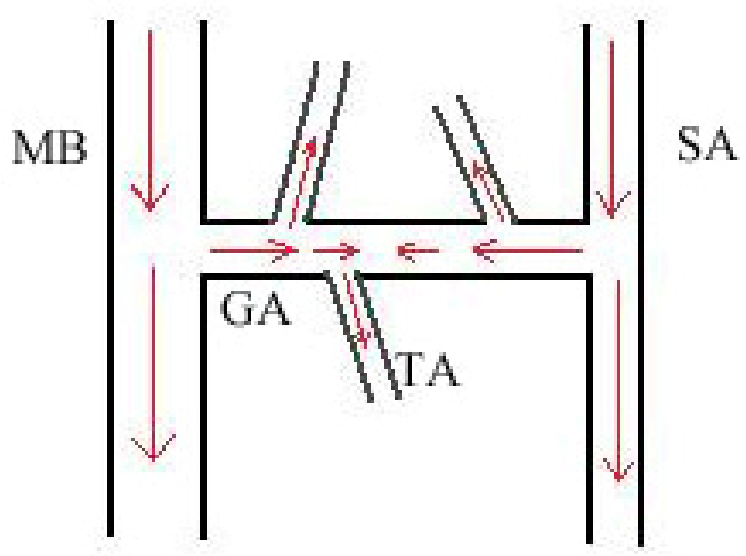

Figure 13. Schematic representation of the arcade structure observed in the mouse gracilis muscle. MB muscular branch, SA - saphenous artery, GA - gracilis artery, TA - transverse arteriole

It is possible to split this arcade structure and treat it as two separate vascular trees being fed by separate arteries (one being the muscular branch, and the other being the saphenous) because there are segments in the GA that have opposing flow directions and feed different TAs and capillaries. The angle with which the transverse arteriole separates from the gracilis artery was used to determine the direction of flow into that arteriole as illustrated in (Figure 14). The split point was determined to be between two arteriole segments with opposing flow directions. All vessels to the left of the split point were assumed to be fed by the muscular branch, and all vessels to the right of the split point were assumed to be fed by the saphenous branch. 


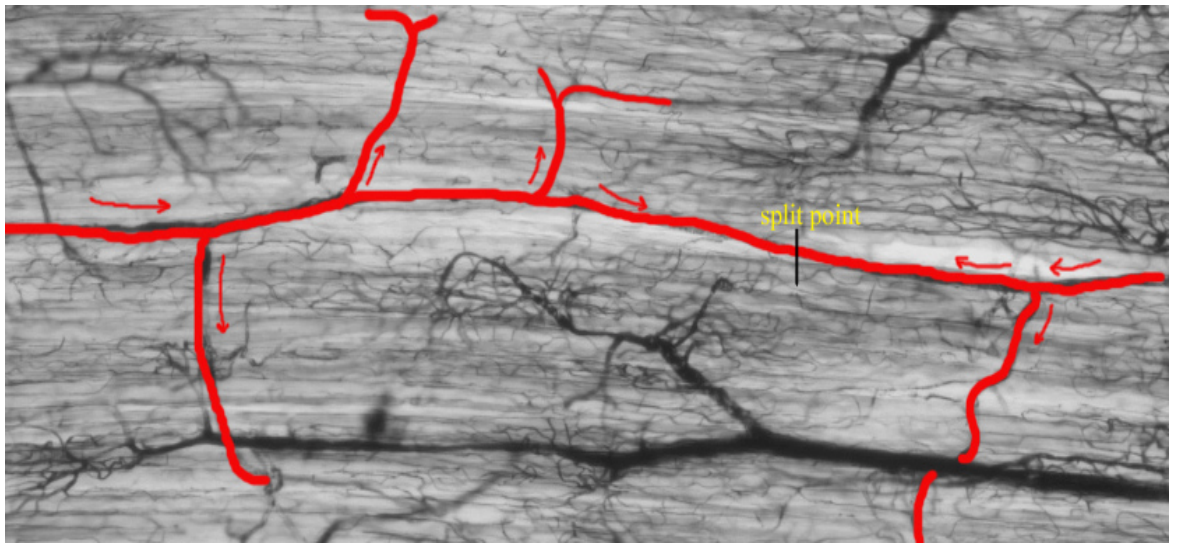

Figure 14. Flow direction in the transverse arterioles were determined by the angle with which they separated from the main artery. The red arrows indicate flow direction in the arterioles.

Labeling was done on Adobe Photoshop 4.0 using the Strahler ordering scheme (Jiang, Kassab et al. 1994). The smallest discernible vessels on a transverse arteriole branch were labeled order 1 . Where two order 1 vessels came together, the parent vessel was labeled order 2. Similarly, when two order 3 vessels came together, the parent vessel was labeled order 3 . In situations where two vessels of unequal orders came together, the parent vessel retained the value of the higher order vessel. For example, an order 2 vessel coming together with an order 3 vessel resulted in a parent vessel of order 3. The following (Figure 15) illustrates the Strahler ordering scheme:

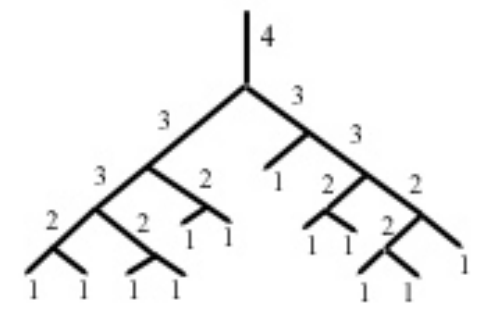

Figure 15. depicts Strahler ordering 
In addition to being assigned orders, each vessel segment was assigned an individual label that could be used for referencing during conversion of the segmental network to an elemental one as well as during hemodynamic calculations. The conversion from segments to elements will be discussed shortly in this section.

Image $\mathbf{J}$ software was used to measure the diameters and lengths of all the vessel segments. The resolution was approximately $0.5 \mu \mathrm{m}$ per pixel. The transition from "vessel" to "non-vessel" was gradual in the images (often several gray pixels), and led to measurement error, especially in the diameter measurements of smaller vessels. Another source of error was the lack of visibility of vessel branches that left the plane of the image and could not be included in the calculations. The following table shows an example of the data obtained after performing morphological analysis on an image.

Table 2. shows an example of data obtained through morphological analysis

\begin{tabular}{|r|r|r|r|r|}
$\begin{array}{c}\text { Segment } \\
\text { label }\end{array}$ & $\begin{array}{c}\text { Parent } \\
\text { label }\end{array}$ & \multicolumn{1}{|c|}{ Order } & $\begin{array}{c}\text { Length } \\
\text { (microns) }\end{array}$ & $\begin{array}{c}\text { Diameter } \\
\text { (microns) }\end{array}$ \\
\hline 2 & 1 & 2 & 197.6 & 15.1 \\
3 & 2 & 2 & 16.2 & 12.9 \\
4 & 2 & 1 & 50 & 10.3 \\
5 & 3 & 1 & 53.9 & 5 \\
6 & 3 & 1 & 65.6 & 5.4 \\
7 & 1 & 5 & 121.3 & 31.6 \\
8 & 7 & 4 & 561.4 & 18.4 \\
9 & 8 & 1 & 16.8 & 7.4 \\
10 & 8 & 4 & 149.2 & 18 \\
11 & 10 & 3 & 170.8 & 16 \\
12 & 10 & 3 & 159.4 & 13.6 \\
13 & 12 & 2 & 246.5 & 13.5 \\
14 & 12 & 3 & 100.9 & 13.1 \\
15 & 14 & 2 & 168.6 & 13 \\
\hline
\end{tabular}


The GA is fed by the MB and SA. However, these two feeding arteries were not included in the analysis as the entire length of these vessels is not visible in the images and hence, a proper estimate of their conductance cannot be made.

\section{Calculations pertaining to morphological models}

\section{Segment reordering}

The initial ordering of the vessels is based on the Strahler scheme in which connectivity solely determined the orders of vessel segments. Thereafter, reordering was performed based on the diameter-defined Strahler system to ensure there was no overlap in the diameters of vessels of different orders. The following equation was used for reordering of vessel segments

\section{(Equation 3):}

$$
\begin{aligned}
& D_{1(n)}^{\prime}=\left[\left(D_{n-1}+S D_{n-1}\right)+\left(D_{n}-S D_{n}\right)\right] / 2 \text { (upper limit) } \\
& D_{2(n)}^{\prime}=\left[\left(D_{n}+S D_{n}\right)+\left(D_{n+1}-S D_{n+1}\right)\right] / 2 \text { (lower limit) }
\end{aligned}
$$

Equation 3. shows the calculation of the upper and lower diameter limits for order $\mathbf{n}$

The mean, $\mathrm{D}_{\mathrm{n}}$ and standard deviation, $\mathrm{SD}_{\mathrm{n}}$ for diameters were calculated for vessels within each order $n$ after the initial ordering. Then the equation shown above was used to revise the orders. After revision, the new values of $\mathrm{D}_{\mathrm{n}}$ and $\mathrm{SD}_{\mathrm{n}}$ were computed and the process was repeated until convergence was obtained. A total of four such iterations were required to obtain convergence in all the 10 muscle samples analyzed.

\section{Combining segments into elements}

An elemental representation of the architecture of a vascular network is more accurate compared to a segmental one. A segments based analysis of a vascular network is useful for hemodynamic analysis but does not provide an accurate representation of the lengths and quantities of vessels. Conversion of segments to elements involves joining together segments of 
the same order connected together in series to form one element. The following figure (Figure

16) represents the conversion of segments to elements:

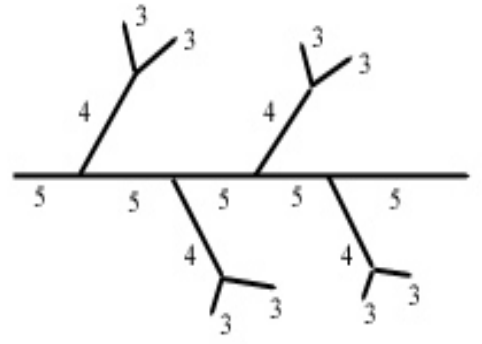

Segments

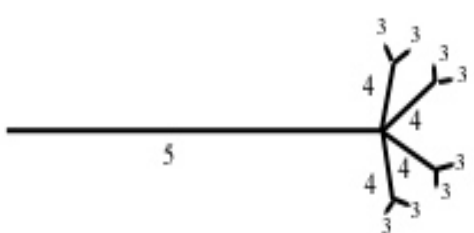

Elements

Figure 16. shows the conversion of a segmental representation of a network to an elemental one

A comparison of the elemental representations of the normal and remodeled networks affords a better assessment of the morphological differences between the two networks relative to a segmental comparative analysis.

Flow, pressure, and wall shear stress computations

\section{Boundary conditions and assumptions}

The pressures at the outlet and inlet were assumed constant and set at $77 \mathrm{mmHg}$ and 30 $\mathrm{mmHg}$ respectively (Pries, Secomb et al. 1995). Blood viscosity was assumed to be constant and was set at $3 \mathrm{cP}$ (Gruionu, Hoying et al. 2005). It was assumed that the vessel walls are nondistensible.

\section{Generation of Linear Equations}

Linear equations were set up in order to calculate pressures and flows. In order to solve a set of linear equations, there should be the same number equations as the number of unknowns. The unknowns are the pressures at the nodes (at segment inlet) and the flows through the 
segments (at segment outlet). These equations are generated based on Hagen-Poiseuille's relationship between flow and pressure (Equation 4) and conservation of mass at each node

\section{(Equation 5).}

$$
Q=\frac{\Delta P \pi D^{4}}{128 \eta l}
$$

Equation 4. Hagen-Poiseuille's equation, in which $\mathbf{Q}, \Delta \mathbf{P}, \mathrm{D}, \boldsymbol{\eta}$, and I represent flow, pressure gradient, radius of the vessel, fluid viscosity, and length of the vessel, respectively. (Kaul and Jayaweera 2006)

$$
\sum Q_{i}=\mathbf{0}
$$

Equation 5. Conservation of Mass equation in which, $Q_{i}$ represents flow through node i. (Kassab, Berkley et al. 1997)

The equations for flow and pressure for a node within an example network is described below. The equations are for node 2 in the network shown in Figure 17. Details on the generation of flow and pressure equations in Excel are included in the Appendix.

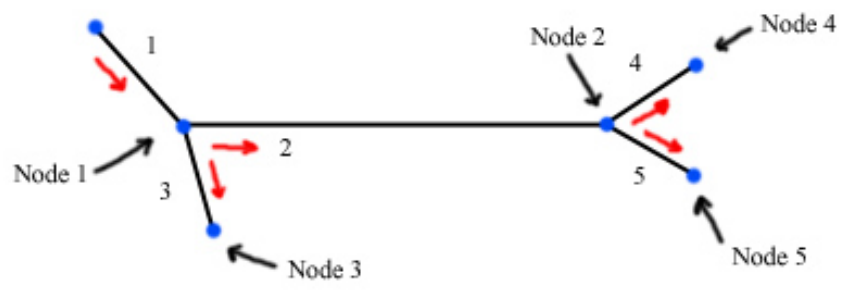

Figure 17. shows an arbitrary network branch with labeled nodes and flow directions (in red).

Equations for node 2:

Pressure equation: $(\mathrm{P} 1-\mathrm{P} 2) * \mathrm{C} 2-((\mathrm{P} 2-\mathrm{P} 4) * \mathrm{C} 4+(\mathrm{P} 2-\mathrm{P} 5) * \mathrm{C} 5)==0$,

Flow equation: $\mathrm{q} 2-(\mathrm{P} 1-\mathrm{P} 2) * \mathrm{C} 2==0$, 
Where P1 represents pressure at node 1, C2 represents the conductance of vessel 2, q2 represents the volumetric flow through vessel 2, and so on. Equation 1 uses conservation of mass, i.e. flow in - flow out $=0$, while equation 2 uses the Hagen-Poiseuille relationship to compute flow through vessel 2. Vessel 2's parent is vessel 1, and its offspring vessels are 4 and 5.

Wall shear stress was calculated using the following relation,

$$
\tau_{\mathrm{w}}=\left(\frac{4 \mu}{\pi}\right) \frac{\mathrm{Q}}{\mathrm{R}^{3}}
$$

Equation 1. Wall shear stress equation

\section{Relationship between wall shear stress, volumetric flow rate $Q$, viscosity $\mu$ and radius $R$}

The calculated flow value for each vessel segment and its diameter were used to calculate the wall shear stress. The viscosity of blood was assumed to be $3 \mathrm{cP}$ throughout the network.

\section{Statistical analyses}

Student's t-test was used to make comparisons between control and remodeled morphology and hemodynamics. ANOVA was used to compare control, remodeled, and doublesided remodeled flow characteristics. 


\section{Results}

Following are some images of the hindlimb vasculature of the mouse gracilis muscle in normal and ischemic tissue (Figure 18, Figure 19). The following images show 2 of the 10 images of the mouse gracilis muscle microcirculation analyzed in this report. Both normal and the corresponding remodeled microcirculation images have been obtained from the two hindlimbs of the same mouse.

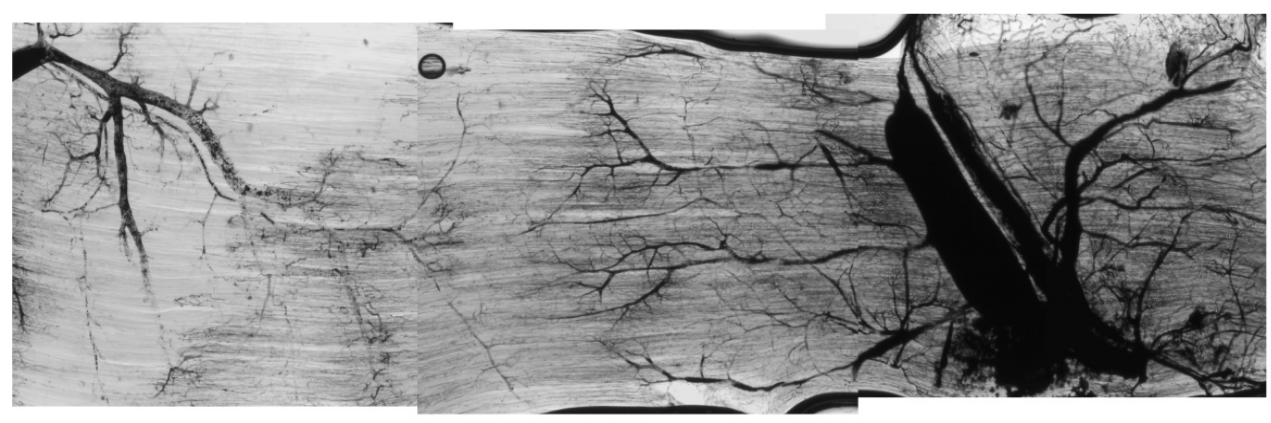

Figure 18. Normal ink-stained vasculature of mouse gracilis muscle. A montage of three images was used to create a view of the entire portion of the microcirculation studied in this report.

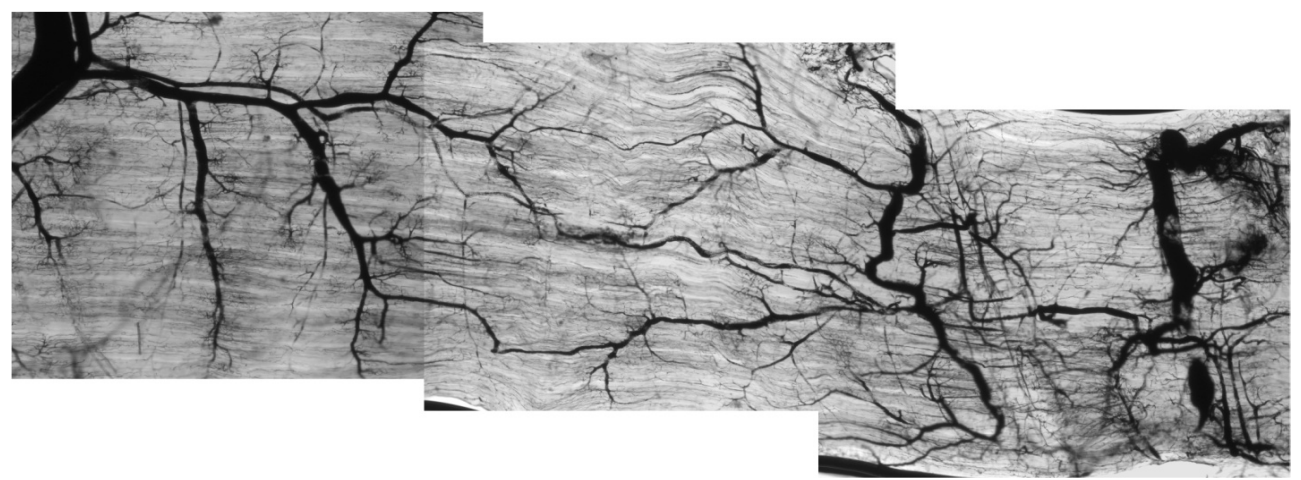

Figure 19. Remodeled ink-stained vasculature of mouse gracilis muscle. A montage of three images was used to create a view of the entire portion of the microcirculation studied in this report. 


\section{Morphological analysis}

Comparison of network dimensions and topology

The average diameter, quantity, and lengths of vessels across the various orders in the normal and remodeled networks indicate the basic differences and similarities between the morphologies of the two types of networks. Given the arcade structure of the microcirculation in the gracilis muscle, it is helpful to keep in mind that the segments of the gracilis artery and the transverse arteriole segments branching immediately off it primarily constitute the order 4 and 5 vessels. Vessels of order 2 and 3 are located mostly on the transverse arterioles, while order 1 vessels are the terminal vessels (most of which are the capillaries, and few are small arterioles beyond which the circulation could not be captured in the analysis due to limitations in imaging). It is also important to note that for the purpose of this analysis, the diameter bounds of the orders of control network vessels were imposed on the corresponding remodeled network vessels so that comparisons in morphology and hemodynamics could be made between vessels of similar caliber. 


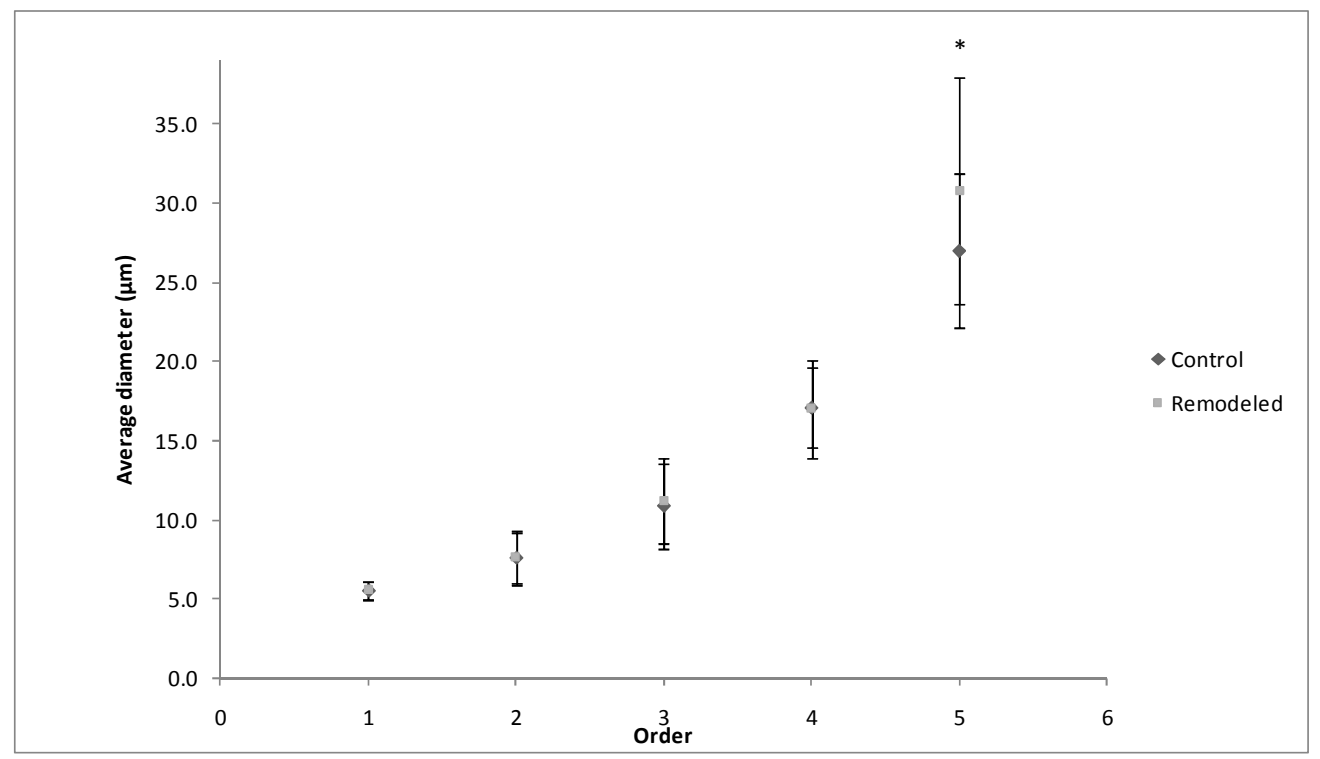

Figure 20. Average diameter of vessels across different orders in normal and remodeled microvasculature.

Bars represent standard deviation. (*) indicates statistical difference between control and remodeled.

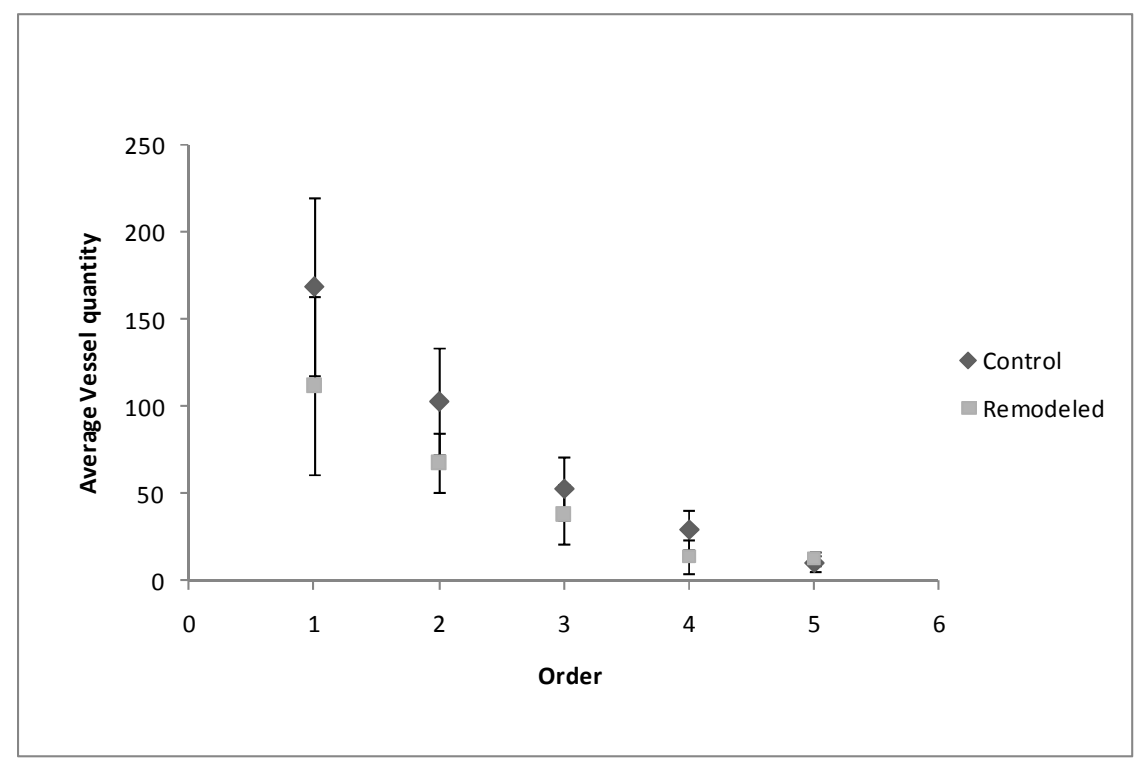

Figure 21. Average quantity of vessels across different orders in normal and remodeled microvasculature.

Bars represent standard deviation. (*) indicates statistical difference between control and remodeled. 


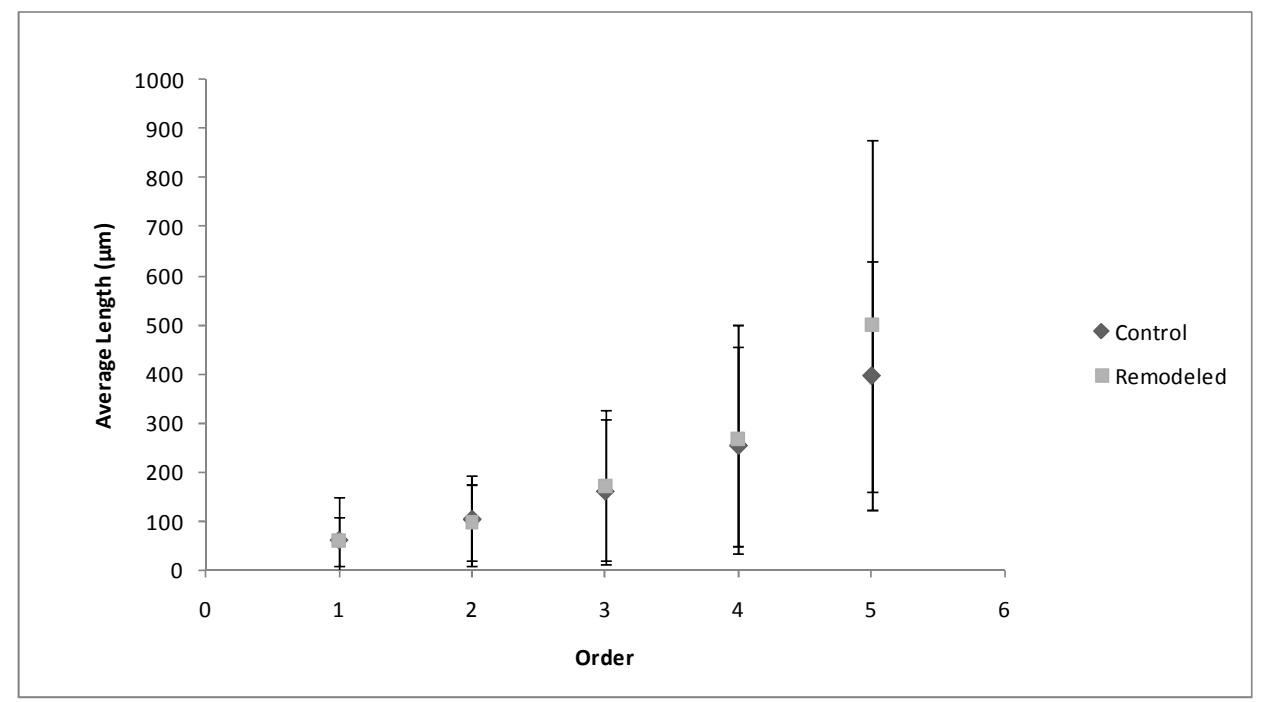

Figure 22. Average length of vessels across different orders in normal and remodeled microvasculature. Bars represent standard deviation. (*) indicates statistical difference between control and remodeled.

The order 1 vessels in both the control and remodeled networks have similar dimensional characteristics such as similar average diameter, length, and quantity of vessels. The average diameter and length of the order 1 vessels being similar is consistent with our expectation since order 1 vessels are mostly capillary vessels that share similar structural and functional properties. It is interesting that after remodeling, the networks consist of a similar number of terminal vessels. Overall, the average quantity of vessels drops throughout all the orders, however, the differences are not statistically significant. There is a significant difference in the diameter of the order 5 control vessels compared to the remodeled. 


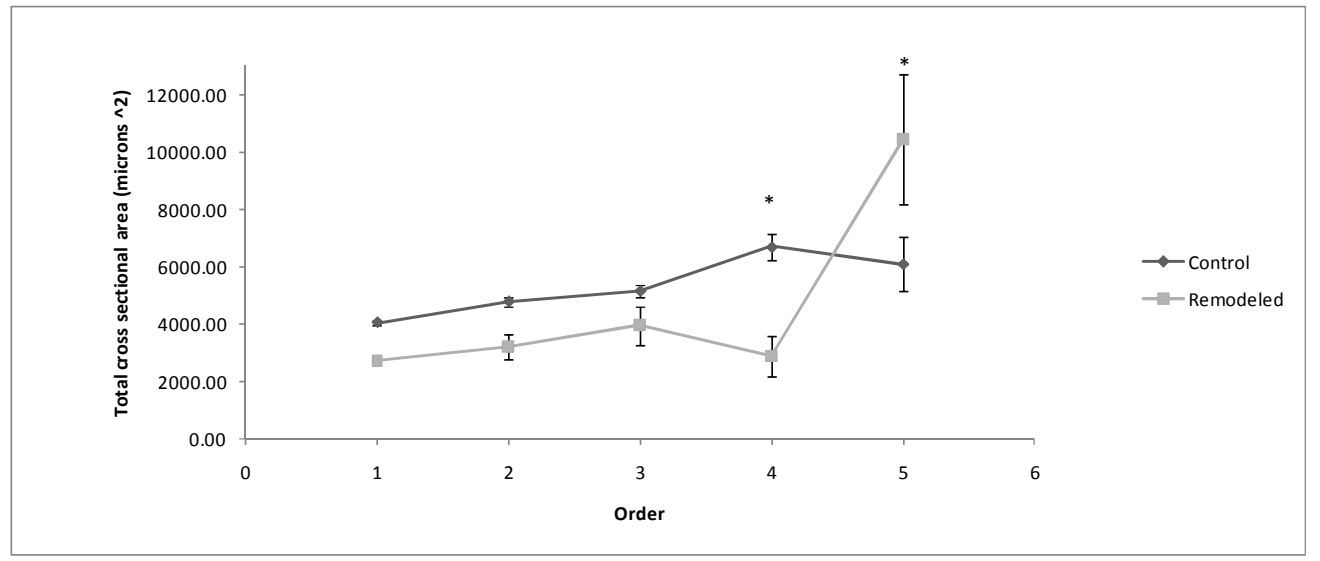

Figure 23. Total cross sectional area of each order for segments. Bars represent standard error. $(*)$ indicate statistical difference $(\mathbf{p}<0.05)$.

The total cross-sectional area (TCA) of vessels shows an overall increasing trend with increasing order in both the normal and remodeled networks. The average TCA of order 4 drops abruptly in the remodeled networks, which is probably due to the significant decrease in the quantity of order $4 \mathrm{~s}$ in the remodeled networks. An important difference between the TCA of the vessels in the segmental and elemental representations is discussed later in this section where the elemental representation of vessel network morphology is presented. 
Comparisons in architecture between normal and remodeled microvasculature Network Connectivity characteristics

After ordering the network, the trends in morphological characteristics among the different orders were analyzed. Horton's law states that the branching ratio from one order to the next will tend to approach a constant through the series (Fenton and Zweifach 1981). If a network obeys Horton's law, the diameter, quantity, and length grow as a geometric sequence as the order number increases. Horton's law applied to diameter:

$$
\frac{D_{n}}{D_{n-1}}=R_{D}
$$

Equation 6. Horton's Law applied to diameter.

$D_{n}$ is the average diameter of vessels of order $n . L_{n}$ and $N_{n}$ can be substituted in place of $D_{n}$ to calculate the ratios for length and quantity, respectively.

In Table 3, each segment of order $n$ in the control vasculature has a diameter that is 1.52 times larger than a segment of order $n$ - 1 . On average the quantity ratio $\mathrm{R}_{\mathrm{N}}$ is larger in the remodeled than in the normal microcirculation and the difference in the ratios for control and remodeled is statistically significant $(\mathrm{p}<0.05)$. However, the difference in the diameter ratios $\left(R_{D}\right)$ and length ratios $\left(R_{L}\right)$ between control and remodeled are not statistically significant $(\mathrm{p}>0.05)$.

Table 3. Number, diameter, and length ratios computed according to Horton's Law. The number ratios between control and remodeled are statistically different from each other. $\left.{ }^{*}\right)$ indicates statistical difference between control and remodeled values $(\mathbf{p}<0.05)$.

\begin{tabular}{ccccc}
\hline & \multicolumn{2}{c}{ CONTROL SEGMENTS } & \multicolumn{2}{c}{ REMODELED SEGMENTS } \\
RATIO & AVERAGE & SD & AVERAGE & SD \\
\hline${ }^{*} \mathrm{R}_{\mathrm{N}}$ & 0.52 & 0.26 & 0.72 & 0.56 \\
$\mathrm{R}_{\mathrm{D}}$ & 1.52 & 0.31 & 1.57 & 0.46 \\
$\mathrm{R}_{\mathrm{L}}$ & 1.61 & 0.74 & 1.76 & 0.69
\end{tabular}


The diameter, length and quantity of vessels vary logarithmically with order, as is consistent with networks that follow Horton's law (Figure 24, Figure 25, Figure 26).

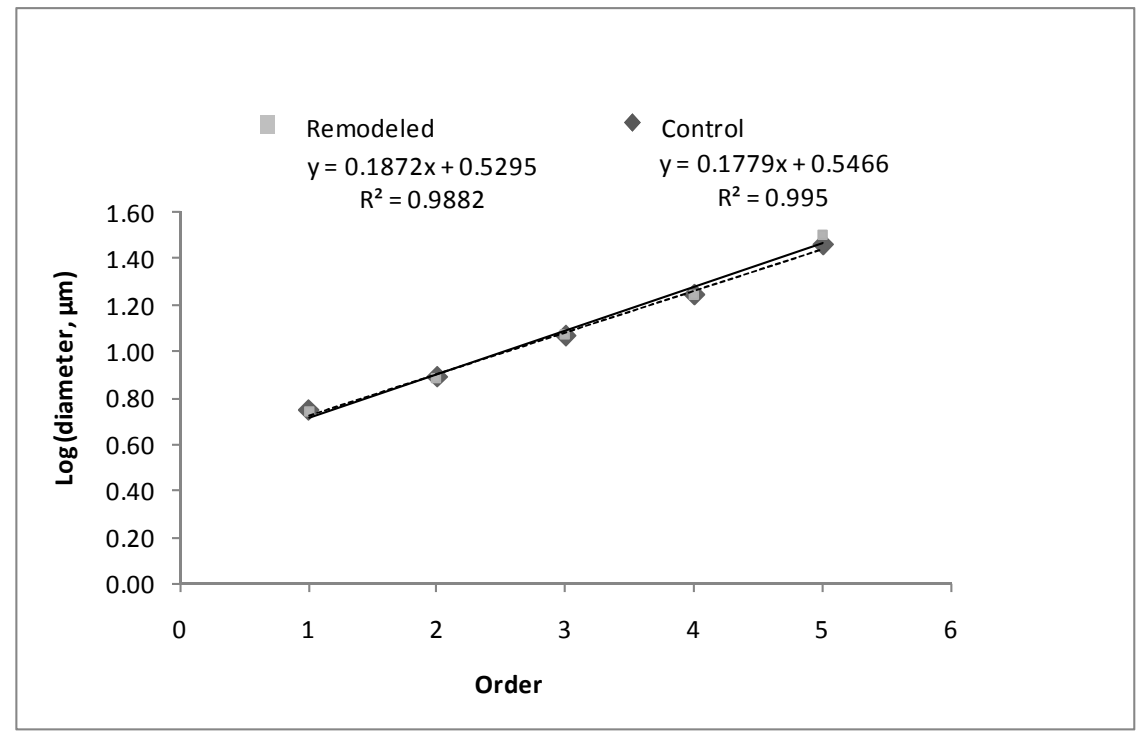

Figure 24. Order versus $\log _{10}$ of vessel diameter for normal and remodeled segments.

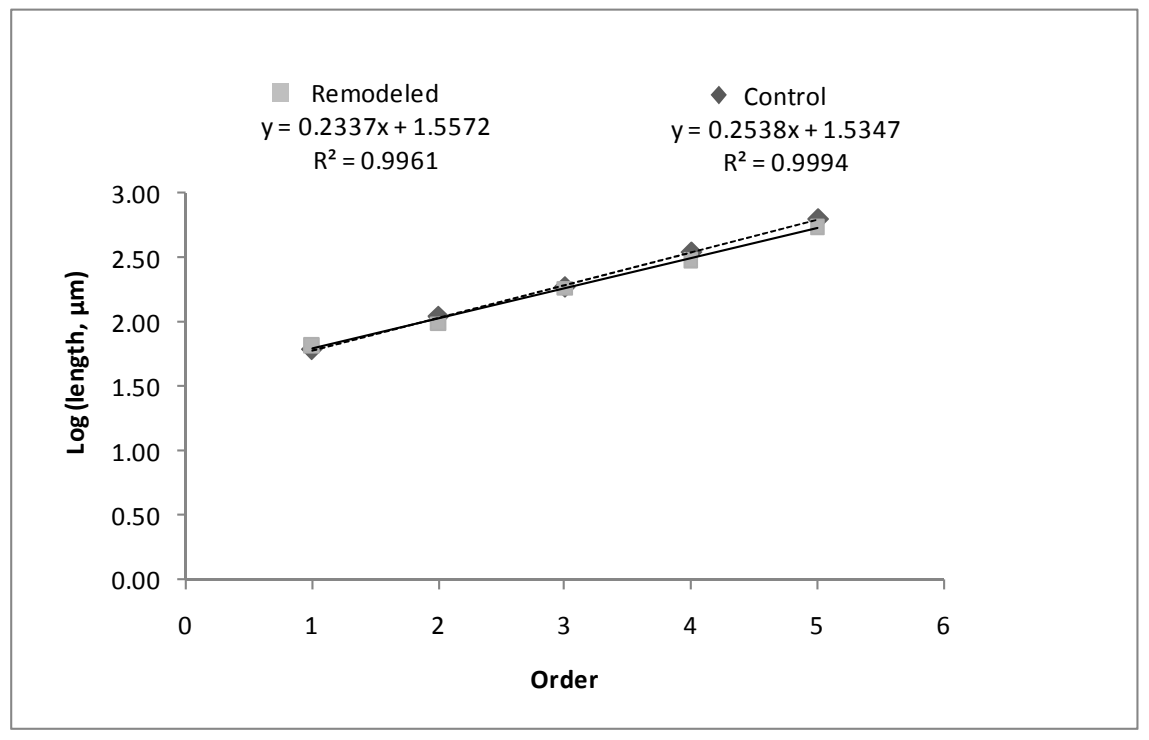

Figure 25. Order versus $\log _{10}$ of vessel length for normal and remodeled segments. 


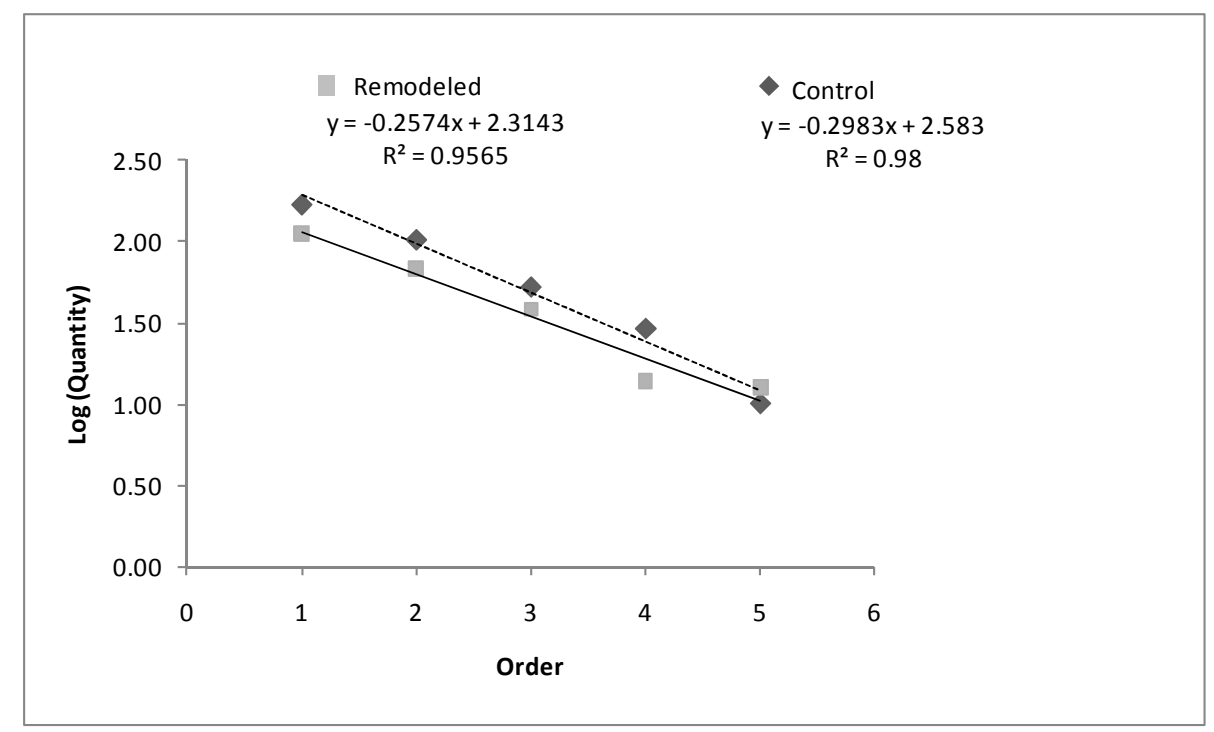

Figure 26. Order versus $\log _{10}$ of vessel quantity for normal and remodeled segments.

The next step in analyzing the connectivity of the segments in the network was to create connectivity matrices that would show the branching pattern across different orders in the normal and remodeled networks. Not always does an order $n$ vessel spring from an order $n+l$ vessel. There are places in the network where order $n$ vessels arise from order $n+2, n+3$, or other higher order vessels. Connections between non-adjacent orders are not captured by Horton's Law. However, connectivity matrices provide a concise representation of all the different types of connections between segments of different orders in the network.

Statistically significant differences in connectivity between the normal and remodeled vessel networks were found between the average number of order 4 vessels giving rise to other order $4 \mathrm{~s}$, and the average number of order $5 \mathrm{~s}$ giving rise to order $4 \mathrm{~s}$, in the analysis of the 5 pairs of muscle samples studied in this report. Thus, significant changes in connectivity occurred only in the higher order vessels. 
Control and Remodeled connectivity

\begin{tabular}{|c|c|c|c|c|c|c|c|c|c|c|}
\hline \multicolumn{11}{|c|}{ Parent (n) } \\
\hline \multirow{6}{*}{ 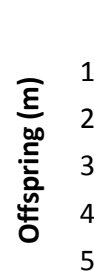 } & $1 C$ & $1 \mathrm{R}$ & $2 C$ & $2 R$ & $3 C$ & $3 R$ & $4 C$ & $4 \mathrm{R}$ & $5 C$ & $5 R$ \\
\hline & $33 \%$ & $33 \%$ & $53 \%$ & $49 \%$ & $13 \%$ & $15 \%$ & $2 \%$ & $4 \%$ & $0 \%$ & $0 \%$ \\
\hline & $0 \%$ & $0 \%$ & $45 \%$ & $42 \%$ & $43 \%$ & $46 \%$ & $11 \%$ & $9 \%$ & $1 \%$ & $3 \%$ \\
\hline & $0 \%$ & $0 \%$ & $0 \%$ & $0 \%$ & $51 \%$ & $54 \%$ & $43 \%$ & $31 \%$ & $5 \%$ & $15 \%$ \\
\hline & $0 \%$ & $0 \%$ & $0 \%$ & $0 \%$ & $0 \%$ & $0 \%$ & $64 \%$ & $44 \%$ & $31 \%$ & $55 \%$ \\
\hline & $0 \%$ & $0 \%$ & $0 \%$ & $0 \%$ & $0 \%$ & $0 \%$ & $0 \%$ & $3 \%$ & $79 \%$ & $89 \%$ \\
\hline
\end{tabular}

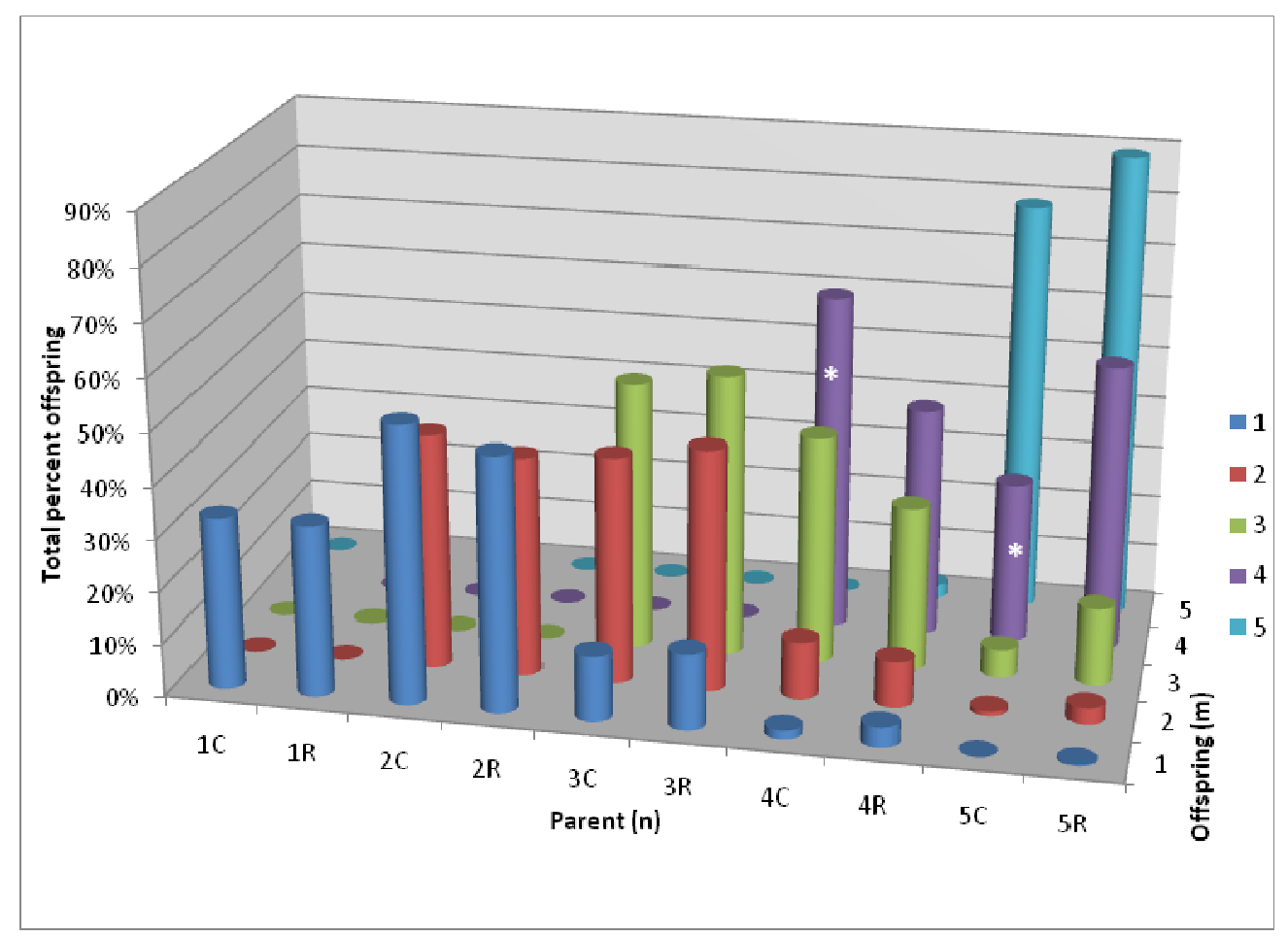

Figure 27. Tabular and graphical representation of connectivity of vessel segments in the normal and remodeled vascular networks. Values describe percent of offspring vessels (m) arising from parent vessels (n). (C) represents control vessels, while (R) represents remodeled vessels, for example, 1C indicates order 1 control segments, and $1 \mathrm{R}$ indicates order 1 remodeled segments. 


\section{Comparison of normal and remodeled hemodynamic characteristics}

\section{Flow and Pressure properties}

Hagen-Poiseuille's relationship between volumetric flow and pressure gradient was used to compute flows and pressure drops throughout the networks in the normal and remodeled networks. An inlet pressure of $77 \mathrm{mmHg}$ was assumed at the inlet of the vessels fed by the muscular branch and saphenous artery, at the two ends of the arcade. At the capillary level the pressure was set at $30 \mathrm{mmHg}$. Additionally, some arteriolar branches could not be traced all the way to the capillary level due to imaging limitations, hence capillary pressure was set at the outlet of the best visible terminal vessels (even though they were not capillaries). In the remodeled networks, the inlet pressures in the vessels fed by the muscular branch were assumed to be $77 \mathrm{mmHg}$ just as in the normal networks. The viscosity of blood was set at $3 \mathrm{cP}$ in both types of networks.

The average individual segmental flow rates in the control and remodeled vascular networks were found to be very similar. This indicates that overall, the architectural changes that occurred during the remodeling process, led to maintaining blood flow at normal levels after 2 weeks of remodeling. 


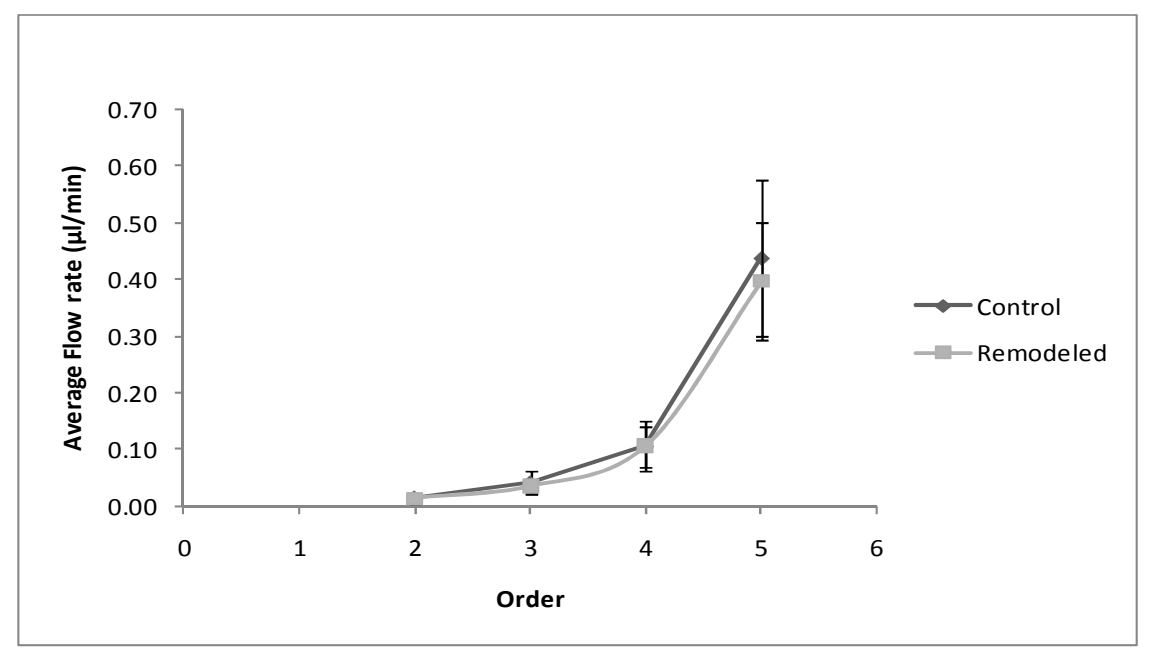

Figure 28. Average flow rate through individual vessel segments. Bars represent standard error.

A major difference between the control and remodeled networks was the loss of blood supply from the saphenous artery in the remodeled networks. An in silico experiment was conducted to quantitatively study the increase in overall blood flow through the network that would occur if the remodeled networks had their blood flow from the saphenous artery restored. The entrance pressure in the vessels of the remodeled network being fed by the saphenous artery was set at $77 \mathrm{mmHg}$ as was done for the control. 


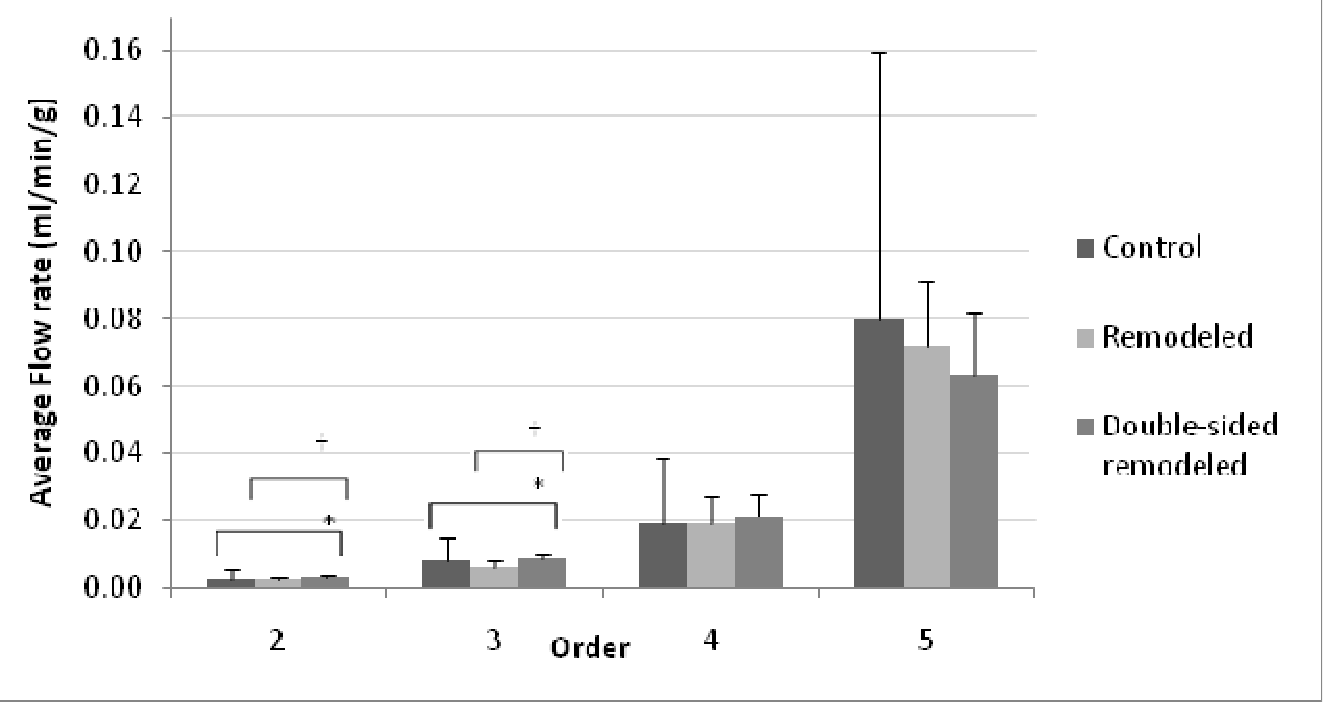

Figure 29. Average overall flow rates across the different orders of vessels in the control, remodeled and hypothetical remodeled networks with flow from both ends of arcade (double-sided remodeled). (*) indicates statistical difference double-sided remodeled and control. $\left({ }^{\dagger}\right)$ indicates statistical difference between doublesided remodeled and remodeled.

The average overall flow rates across the various orders was observed to be higher in all the orders in the remodeled networks with flow restored from the saphenous artery except order 5 , in comparison to the control and remodeled networks. It is interesting that the alterations in the morphological characteristics of the remodeled networks is such that on restoring flow from both sides, the largest order vessels experience a drop in average flow rate relative to those in the remodeled network with single flow supply. Additionally, flow is higher in the lower orders (2, 3 , and 4), with increases in flow being statistically significant in orders 2 and 3.

The increase in average flow in order $4 \mathrm{~s}$ despite lower average flow in order $5 \mathrm{~s}$ can possibly be due to the significantly lower quantity of order $4 \mathrm{~s}$ in the remodeled networks and the 
statistically significant connectivity differences among order $4 \mathrm{~s}$ and $5 \mathrm{~s}$ in the control and remodeled networks. As shown previously in this section of the report, the control networks on average have a higher number of order 4 -order 4 connections and lower order 5 -order 4 connections compared to the corresponding averages in the remodeled networks. Therefore, when flow from the saphenous is restored in silico in the remodeled networks, the percentage of order $4 \mathrm{~s}$ receiving higher flow from order $5 \mathrm{~s}$ is greater than the percentage of order $4 \mathrm{~s}$ receiving higher flow from order $4 \mathrm{~s}$ (since vessels of order 5 are of higher caliber than order $4 \mathrm{~s}$, they transfer a higher volumetric flow rate to the order 4 vessels). Additionally, the remodeled order 5 vessels have a higher average diameter compared to the control order 5 vessels (the difference in their means being statistically significant), which leads to higher conductance of the remodeled order 5 vessels and thus, higher volumetric flow transfer to the order $4 \mathrm{~s}$ in the remodeled compared to the control networks.

By a similar argument it can be expected that the average flow in the order $5 \mathrm{~s}$ in the remodeled networks with double-sided flow must increase, however, it actually decreases. This is most likely due to the lower average diameter of the vessels in the remodeled network that are fed by the saphenous artery. During the remodeling process, the average diameter of the vessels being fed by the muscular branch increases relative to the remodeled. Maximum increase in average diameter is observed in the central portion of the gracilis artery, while the gracilis segments closer to the saphenous side show little or no increase in average diameter compared to the control network. Therefore, when flow from the saphenous side is restored, the segments receiving flow from the main artery are on average order 3 or order 4 vessels or occasionally lower diameter order 5s. Thus, blood flow is comparatively low in order 5 vessels despite restoration of flow from the saphenous side. 
Table 4. Comparison of the total volumetric flow rate through control, remodeled, and double-sided remodeled networks.

\begin{tabular}{c|c|c|c|c|c|c|c|c}
\hline Control & $\begin{array}{c}\text { Flow rate } \\
(\mathrm{ml} / \mathrm{min} / \mathrm{g})\end{array}$ & $\mathrm{SE}$ & Remodeled & $\begin{array}{c}\text { Flow rate } \\
(\mathrm{ml} / \mathrm{min} / \mathrm{g})\end{array}$ & $\mathrm{SE}$ & Double-sided & $\begin{array}{c}\text { Flow rate } \\
(\mathrm{ml} / \mathrm{min} / \mathrm{g})\end{array}$ & SE \\
\hline M1 & 0.19 & 0.14 & M1 & 0.13 & 0.07 & M1 & 0.19 & 0.06 \\
M5 & 0.39 & 0.1 & M5 & 0.24 & 0.09 & M5 & 0.29 & 0.09 \\
M6 & 0.27 & 0.08 & M6 & 0.2 & 0.08 & M6 & 0.32 & 0.08 \\
M7 & 0.2 & 0.06 & M7 & 0.21 & 0.07 & M7 & 0.24 & 0.07 \\
M8 & 0.22 & 0.08 & M8 & 0.23 & 0.1 & M8 & 0.21 & 0.1 \\
Average & $\mathbf{0 . 2 5}$ & $\mathbf{0 . 1 4}$ & Average & $\mathbf{0 . 2}$ & $\mathbf{0 . 1}$ & Average & $\mathbf{0 . 2 5}$ & $\mathbf{0 . 1}$
\end{tabular}

Comparison of the data trend indicates that there is no difference in control and remodeled flows. Upon restoration of flow through the saphenous artery in the remodeled networks, there is no significant change in flow compared to the control and remodeled networks. On comparing the morphology and hemodynamics of vessels in different orders in the control and remodeled networks it can be seen that there are not many statistically significant differences in the average diameter, quantity, connectivity, and lengths of vessels in the two types of networks. However, the individual quantitative impact of the changes in morphological parameters such as diameters and lengths of the vessels is not known. In order to understand how architecture impacts flow it is necessary to determine how the flow would change if the diameters of the vessels were altered or if the connectivity of vessels was changed in the remodeled networks. 


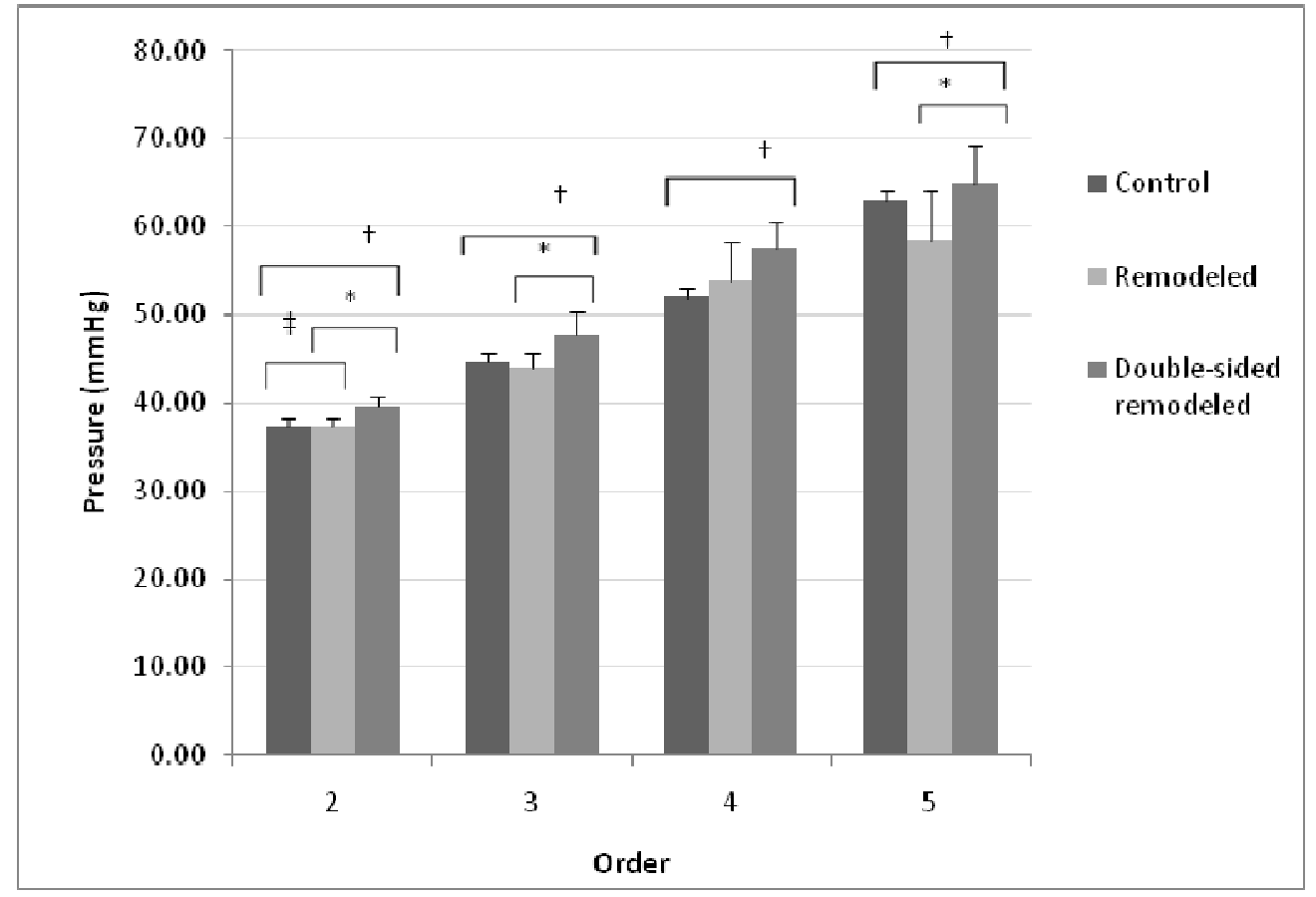

Figure 30. Average pressure in segments of each order. Bars represent standard error. $\left(^{\star}\right)$ indicates statistical difference between control and remodeled. $\left(^{\dagger}\right)$ represents statistical difference between double-sided remodeled and control. $\left(^{*}\right)$ indicates statistical difference between remodeled and double-sided remodeled.

Overall, the control, remodeled, and double-sided remodeled networks showed a drop in average pressure across the networks. The pressures in the remodeled network with double-sided flow supply were higher than those in the control and remodeled networks throughout all the orders. The difference in control and remodeled intravascular pressure was statistically significant only in the order 2 vessels. 


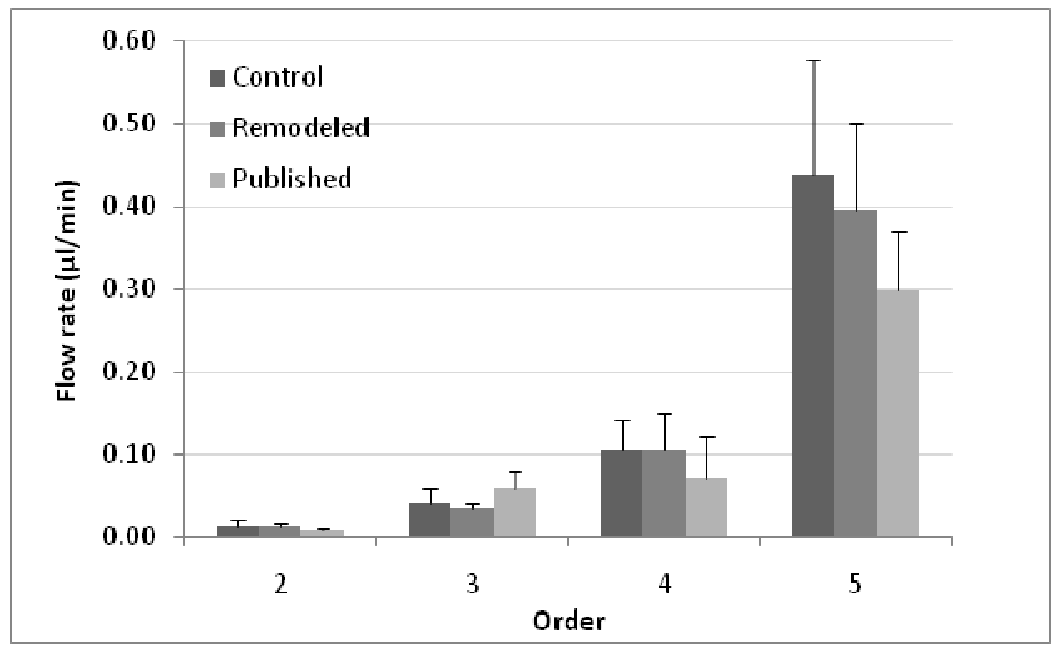

Figure 31. Comparison of calculated (control and remodeled) and published values of individual segmental flow rates. Calculated values are for the average flow values from the control network data. Bars represent standard error.

Overall, the calculated control and remodeled network flow values are comparable to the published values for all the different orders. The published values were obtained from data collected from rat skeletal muscle (Zweifach, Kovalcheck et al. 1981).

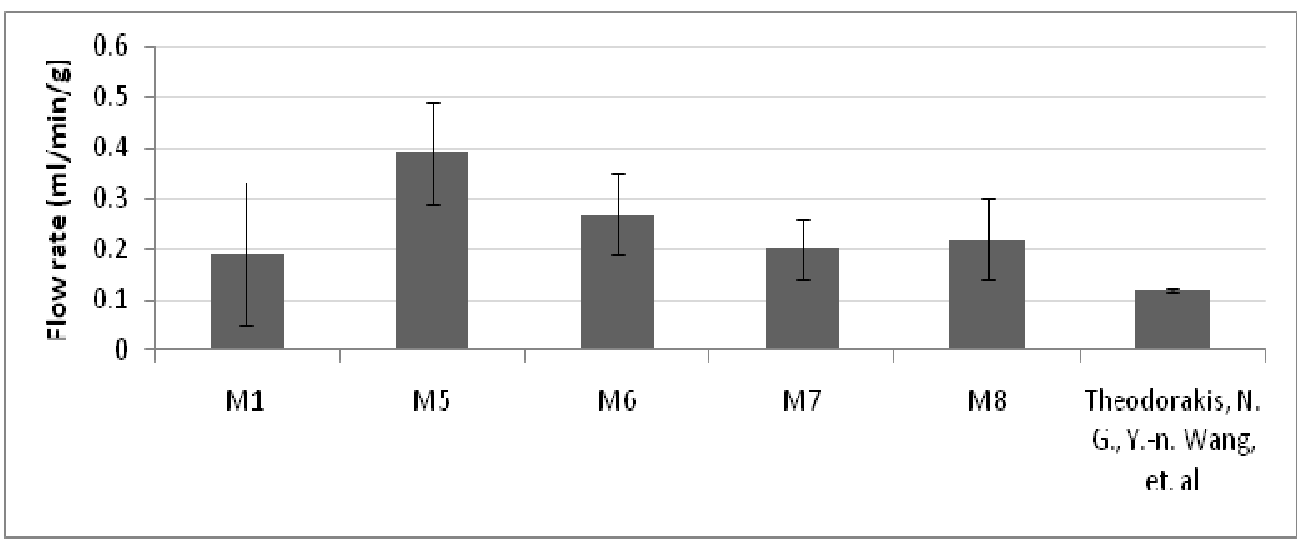

Figure 32. Comparison of overall flow in the control networks with literature. M1, M5, M6, M7, and M8 represent the 5 muscle samples (control only). Bars represent standard error. 
The volumetric flow rates through the control networks are comparable to the value obtained from literature. The literature value was obtained from data collected from the hepatic portal vein of mice.

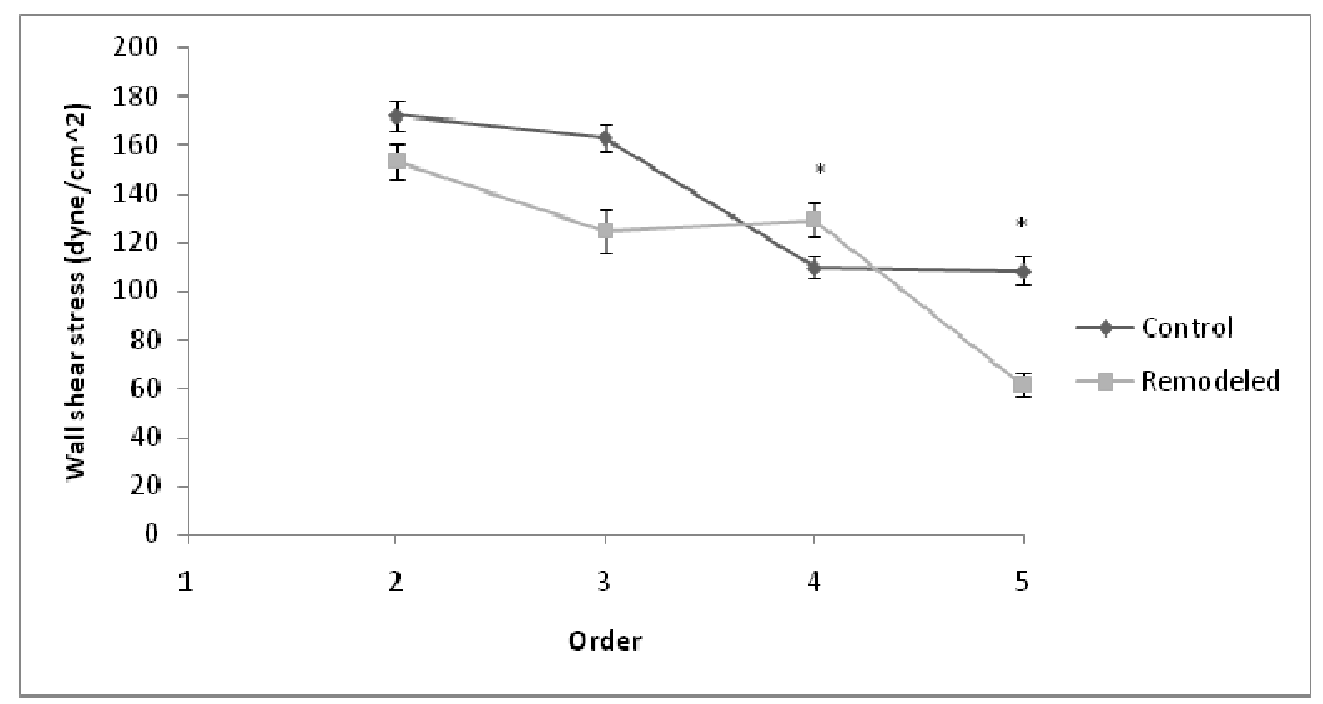

Figure 33. Average wall shear stress in individual vessel segments of different orders. Bars represent standard error. $\left(^{*}\right)$ indicates statistical difference between control and remodeled wall shear stress values.

In comparing the remodeled and normal networks, it is useful to quantitatively determine the forces that act on the walls of vessels, such as wall shear stress, to gain insight on the effect of such forces on network morphology and vice versa. As discussed in the previous chapter, wall shear stress (WSS) is an important stimulus for vascular remodeling. Vessels in the microcirculation have been found to adapt structurally in response to variations in WSS and intravascular pressure as discussed previously in this report. When WSS levels are raised, the vessels increase in luminal diameter to lower the shear stress on the walls. The complete removal of blood supply from the saphenous side of the microcirculation in the gracilis leads to increased flow through the muscular branch and the portion of the gracilis artery that receives its blood 
supply from it. This leads to elevation of shear stress in parts of the gracilis artery where flow rates increase dramatically. Increase in flow leads to shear stress elevation which triggers collateral enlargement of the gracilis artery/longitudinal arteriole. In the images of the remodeled network studied in this report enlargement of the gracilis artery near the muscular branch was observed. Consistent with this observation is the decrease in WSS observed in the order 5 vessels which mainly consist of vessel segments of the gracilis fed by the muscular branch. The WSS is expected to be lower in the order $5 \mathrm{~s}$ of the remodeled versus those in control, because for the same inlet and outlet pressures of $77 \mathrm{mmHg}$ and $30 \mathrm{mmHg}$ respectively, and almost identical morphology of the small arterioles and capillaries in both the control and remodeled networks, the remodeled vessels of larger caliber would have lower flow rates, and thus lower WSS.

The average WSS in the remodeled order 4 vessels however, is higher than that in the control, with the difference between the means being statistically significant. This is possibly due to the significant increase in the average ratio of order 5-order 4 connections in the remodeled networks, which leads to flows from order $5 \mathrm{~s}$ being distributed to a higher percentage of order $4 \mathrm{~s}$ in the remodeled networks relative to the control. 


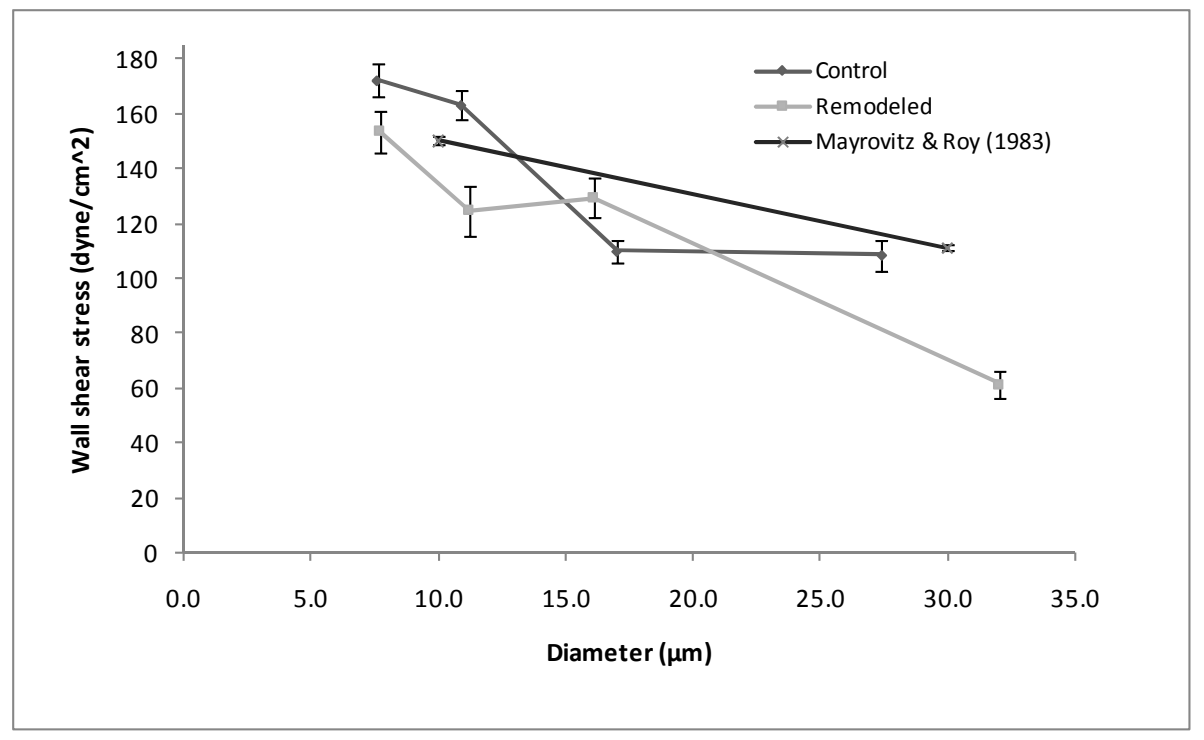

Figure 34. Comparison of calculated wall shear stress (control and remodeled) with literature. Bars represent standard error.

The calculated WSS values in the lower diameter range $(5-10 \mu \mathrm{m})$ and upper diameter range $(25-30 \mu \mathrm{m})$ in the control and remodeled networks are comparable to literature values.

Typically, smaller arterioles have higher WSS, whereas larger arterioles have lower WSS due to the inverse dependence of WSS on the third power of vessel diameter.

Table 5. Wall shear stress in the control and remodeled vessels for all orders.

\begin{tabular}{|c|c|l|l|l|l|l|l|l|}
\hline \multicolumn{3}{|c|}{} & \multicolumn{3}{c|}{ REMODELED } \\
\hline Order & $\begin{array}{l}\text { Vessel } \\
\text { Quantity }\end{array}$ & $\begin{array}{l}\text { Average } \\
\text { diameter } \\
(\mu \mathrm{m})\end{array}$ & $\begin{array}{l}\text { WSS } \\
\left(\text { dyne/ cm }{ }^{2}\right)\end{array}$ & SE & $\begin{array}{l}\text { Vessel } \\
\text { Quantity }\end{array}$ & $\begin{array}{l}\text { Average } \\
\text { diameter } \\
(\mu \mathrm{m})\end{array}$ & $\begin{array}{l}\text { WSS } \\
\left(\text { dyne/ } \mathrm{cm}^{2}\right)\end{array}$ & SE \\
\hline 2 & 103 & 7.6 & 172.17 & 6.24 & 68 & 7.7 & 153.47 & 7.53 \\
3 & 53 & 10.9 & 163.19 & 5.42 & 38 & 11.2 & 124.77 & 8.88 \\
4 & 29 & 17.0 & 109.9 & 4.34 & 14 & 16.1 & 129.38 & 7.030 \\
5 & 10 & 27.4 & 108.54 & 5.65 & 13 & 32.0 & 61.72 & 4.86
\end{tabular}




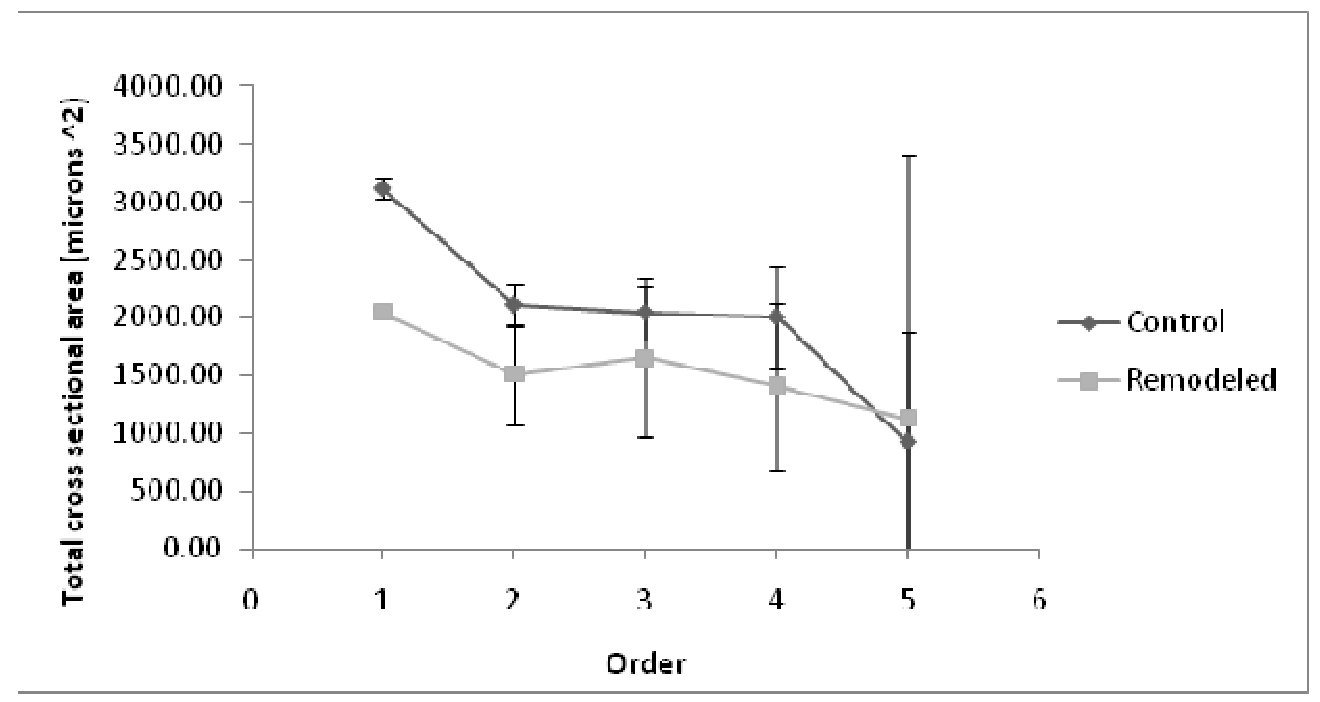

Figure 35. Total cross sectional area of each order for elements. Bars represent standard error.

The TCA of the vessel elements in the control and remodeled networks do not show any statistically significant difference between their means for any of the orders. The comparison of TCA of all vessels within each order shows that elements typically have increasing TCA with increasing order in both control and remodeled networks (Figure 35). The total cross-sectional areas of the smaller vessels must be greater than that of the larger vessels in order to accommodate an equal volumetric flow rate. Since lower order vessels have lower diameters, the TCA of the lowest order must be the highest. TCA was computed by summing the individual cross-sectional areas in each order. When segments were joined into elements, the number of vessels in each order decreased, and thus led to a reduction of the number of cross-sectional areas being included in the computation. This comparison of TCA of control and remodeled 
segments and elements supports the conclusion that elements provide a more realistic representation of the vascular network architecture.

\section{Network connectivity characteristics}

Table 6. Number, diameter, and length ratios computed according to Horton's Law. The length ratios between control and remodeled are statistically different from each other. $\left({ }^{*}\right)$ indicates statistical difference between control and remodeled values $(\mathbf{p}<0.05)$.

\begin{tabular}{ccccc}
\hline \multicolumn{2}{c}{ CONTROL ELEMENTS } & \multicolumn{3}{c}{ REMODELED ELEMENTS } \\
RATIO & AVERAGE & SD & AVERAGE & SD \\
\hline $\mathbf{R}_{\mathrm{N}}$ & $\mathbf{0 . 3 9}$ & $\mathbf{0 . 1 8}$ & $\mathbf{0 . 4 3}$ & $\mathbf{0 . 2 2}$ \\
$\mathbf{R}_{\mathrm{D}}$ & $\mathbf{1 . 3 6}$ & $\mathbf{0 . 4 5}$ & $\mathbf{1 . 5 0}$ & $\mathbf{0 . 8 4}$ \\
R $_{\mathrm{L}}$ & $\mathbf{2 . 1 8}$ & $\mathbf{0 . 9 0}$ & $\mathbf{3 . 8 2}$ & $\mathbf{3 . 4 8}$
\end{tabular}

While the segmental data indicated a significant difference between the quantity of

vessels in adjacent orders of the control and remodeled networks, the elemental data indicates a significant difference between the lengths of vessels in adjacent orders for the same. This can be understood by the fact that the conversion of segments to elements led to a reduction of quantity of vessels in each order and consequently an increase in vessel lengths. On comparing Horton's Law number ratios between segments and elements for control and remodeled data, it is observed that on average a greater proportion of segments are converted to elements in the remodeled data compared to the control (segmental $\mathbf{R}_{\mathbf{N} \text { control }}=\mathbf{0 . 5 2}$, elemental $\mathbf{R}_{\mathbf{N} \text { control }}=\mathbf{0 . 7 2}$ and segmental $\mathbf{R}_{\mathbf{N} \text { remodeled }}=\mathbf{0 . 3 9}$, elemental $\mathbf{R}_{\mathbf{N} \text { control }}=\mathbf{0 . 4 3}$ ). This led to the increase in length of a greater proportion of remodeled vessels compared to control vessels across various orders. This assessment indicates that after the remodeling process, the network ended up with a higher proportion of same order segments connected in series, than in the control network. One of the factors that most likely contributed to this significant increase in the length ratio in the remodeled elemental network is the increase in blood path length in the remodeled networks as flow was along one direction only throughout the gracilis artery. Thus, it is possible that certain 
same order segments that contained flows from opposing sides could come together as one element when flow directions changed in the remodeled networks.

Along with computing the number, diameter, and length ratios for control and remodeled elements, the log plots of vessel quantity, length, and diameter were generated to assess whether these three morphological parameters vary logarithmically with respect to order. The results indicate that overall, the quantity, length, and diameter of vessels vary logarithmically with respect to order in both control and remodeled networks.

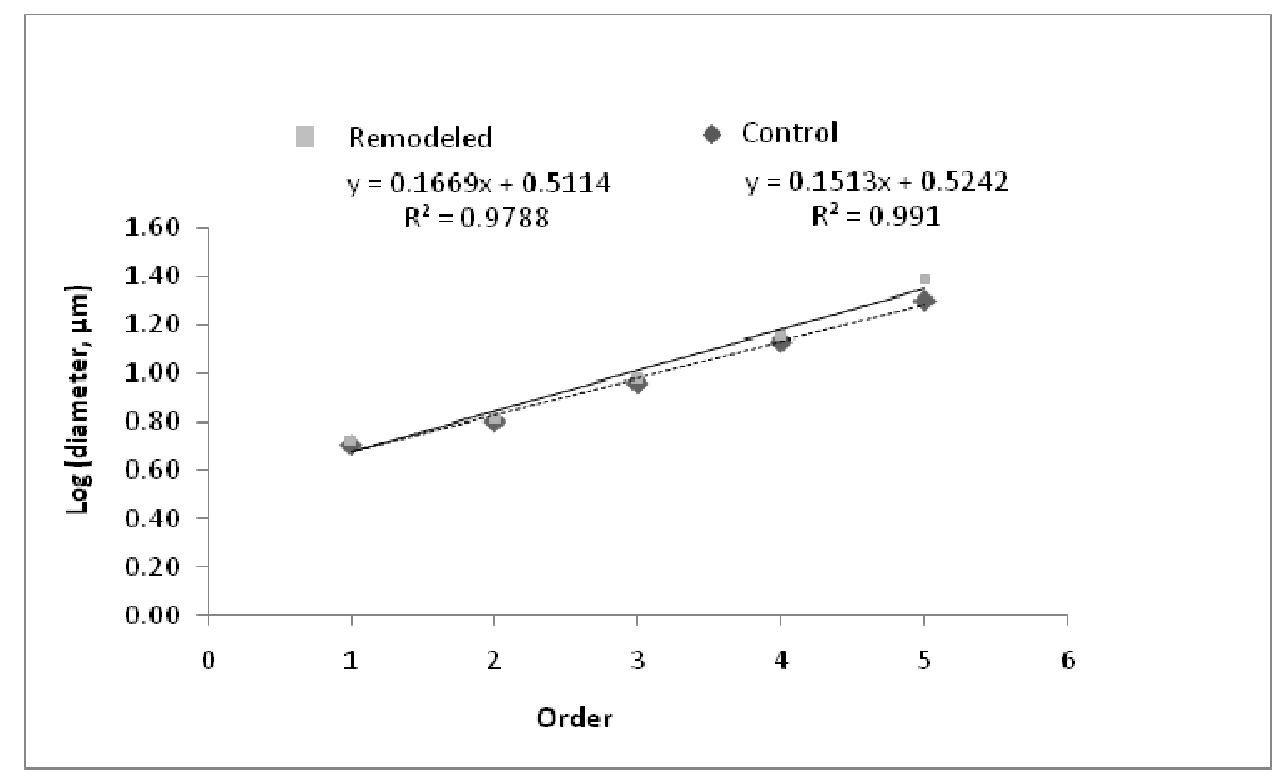

Figure 36. Order versus $\log _{10}$ of vessel diameter for normal and remodeled elements. 


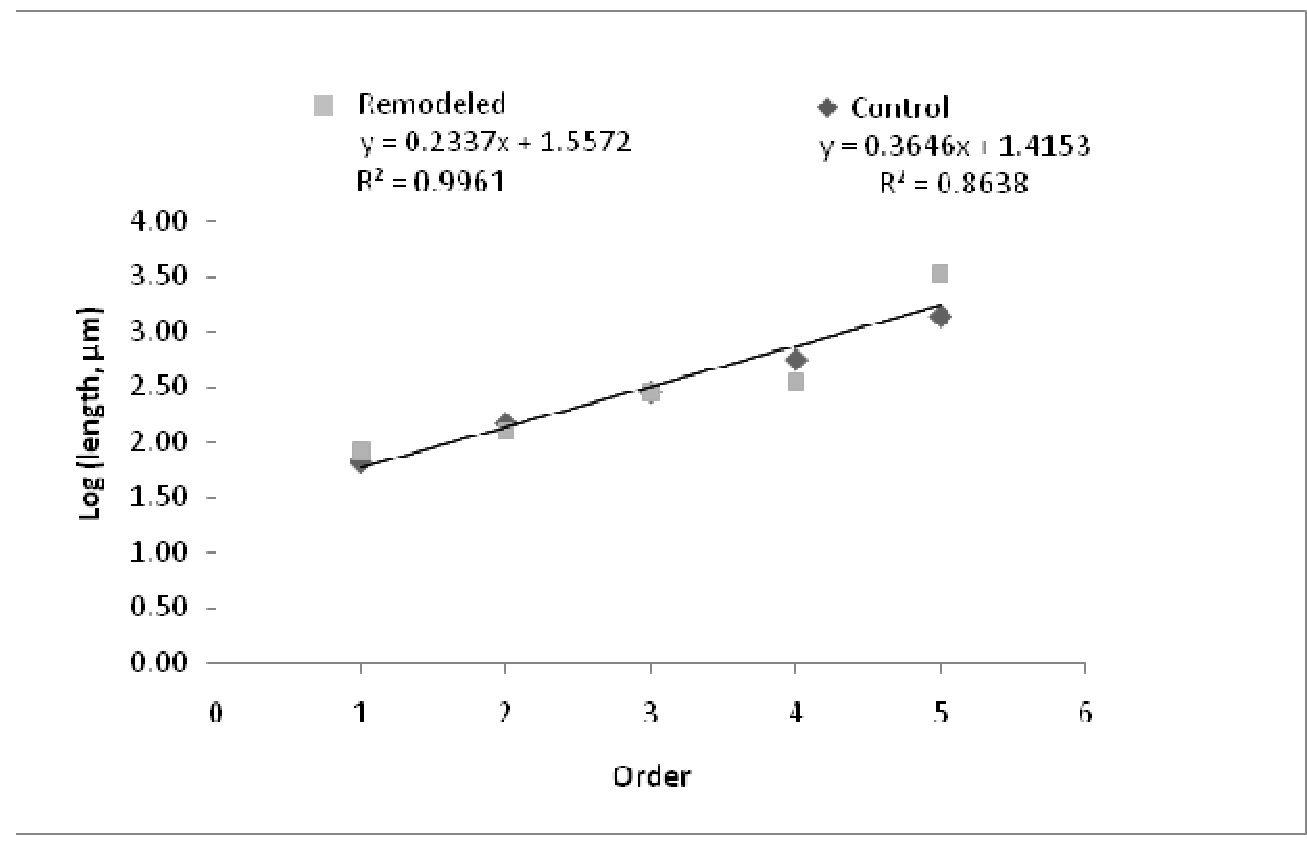

Figure 37. Order versus $\log _{10}$ of vessel length for normal and remodeled elements.

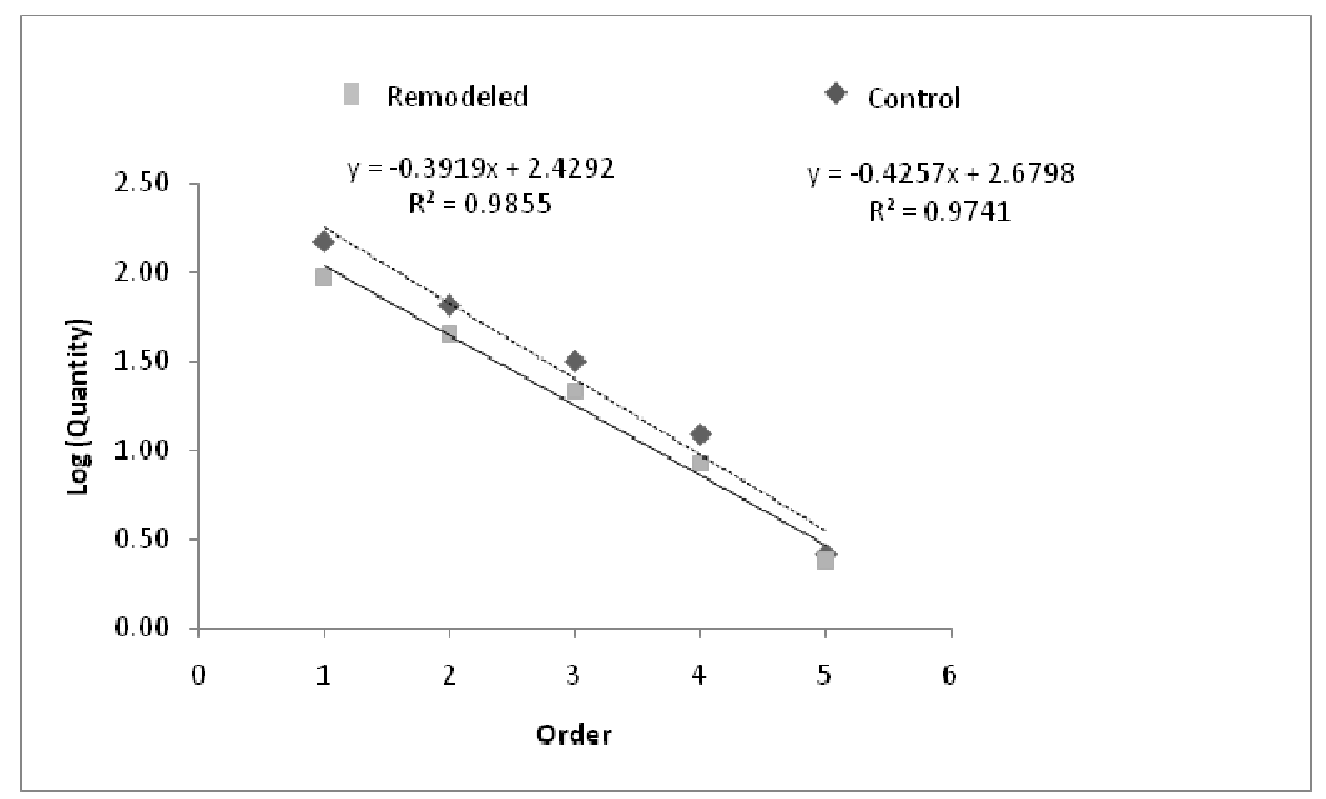

Figure 38. Order versus $\log _{10}$ of vessel quantity for normal and remodeled elements. 


\section{Discussion}

\section{Recap}

Peripheral arterial disease affects a significant portion of the older population and can often lead to further serious health complications (Hershey, Baskin et al. 2001). Two primary changes that occur in the microcirculation post arterial occlusion are reduced vasomotor capacity (ability to alter luminal diameter in response to stimuli) and architectural changes in the vascular network. This paper focuses on the changes in architecture that occur post arterial occlusion and their impact on flow through the network.

Several methods of organizing the architectural information on networks have been previously developed, and simplified models of vascular flow have been used to study the architecture and flow characteristics of vascular networks. The goal of the second chapter in this report was to assess the changes in morphology and flow between normal and remodeled vascular networks, and determine whether sufficient information is revealed to help correlate changes in flow characteristics to variations in morphology. The quantity and dimensions as well network characteristics of the vessel networks as a whole were assessed. Horton's Law, the diameter-defined Strahler ordering scheme, and connectivity matrices were used to organize morphological information regarding both the normal and remodeled networks. Additionally, trends and patterns in morphological features came to light with the use of these methods. The Hagen-Poiseuille relationship between flow and pressure was employed to estimate flow in the individual segments of the networks. The orders assigned to the vessels using the Strahler method allowed grouping various portions of the network based on the diameter and connectivity of vessels, and organizing the flow information with respect to vessel order. 


\section{Morphological analysis}

Morphological analysis involved measuring the architectural features of the vascular networks. In order to obtain the morphological information from the mouse gracilis muscle, images of the ink-filled vasculature in the relevant portion of the gracilis microcirculation were generated. These images provided the contrast necessary to differentiate vascular structures from skeletal muscle fibers. However, some difficulties were encountered in identifying small vessels and measuring vessel diameters and lengths, which will be discussed later in this section.

Overall, both the control and remodeled microcirculation could be captured sufficiently well to be able to study trends in vascular topology in both types of networks, and make statistical comparisons.

\section{Horton's Law, Strahler ordering scheme, and connectivity matrices}

The objective behind using descriptive models used to assess the morphology of the normal and remodeled vascular networks was to organize the structural information about the networks, as well as group the different vessels in the network for effective comparison of anatomical and hemodynamic characteristics. The first step in the entire analysis of the architecture and flow properties of normal and remodeled vascular networks was to assess whether there were any significant changes in the architecture of the networks after remodeling and what they were.

In order to compare vessels of similar caliber, the Strahler ordering scheme was applied on the control networks, and the upper and lower diameter bounds for all the orders was applied to the vessels of the remodeled network. This was done so that each order had the same meaning for both the control and its corresponding remodeled network. 
Overall, the logarithmic trends followed by diameter, length, and quantity of vessels with respect to order in both normal and remodeled networks, was brought to light by the log-plots of the morphological parameters versus order (Figure 24, 25, 26). Based on this assessment it can be concluded that even after remodeling, there was no deviation from Horton's Law in the microvascular topology. The number, diameter, and length ratios also revealed interesting changes that occurred in the networks post-remodeling. For instance, the remodeled networks on average ended up with a larger number of same order segments connected in series compared to the control networks (indicated by significant increase in length ratio $\left(\mathbf{R}_{\mathbf{L}}\right)$ in the remodeled)

(Table 6). This is possibly linked to the observation that there is a trend of decreasing difference in average vessel quantity with increasing order (Figure 21). It is possible that the increase in luminal diameter of vessels during remodeling led to several order 1 vessels transitioning into order $2 \mathrm{~s}$, order $2 \mathrm{~s}$ transitioning into order $3 \mathrm{~s}$ and so on. Thus, several bifurcations in which earlier parent orders were higher than daughter orders could have ended up having one or more daughters with orders equal to that of the parent. Another explanation for the significant increase in the length ratio is the increase in blood path length in the remodeled networks, as discussed in the previous section.

Along with the analysis of trends in diameters, lengths, and quantities of vessels, the assessment of vessel connectivity in the network is important in determining the flow and pressure distribution in the vascular network. The connectivity matrices provided a compact representation of the connections between all the orders in the network. Additionally, the sideby-side comparisons of the percentages of specific connections in both types of networks revealed similarities and differences in vessel connectivity in the control and remodeled networks in a visually convenient fashion. 
The percentage of offspring vessels of order $m$ arising from parent vessels of order $n$ has an impact on the flow rates and pressure gradients in the offspring vessels. Statistically significant differences were observed in the percentages of order 5-order 4 and order 4 -order 4 connections.

Once the differences observed in the morphology of the control and remodeled networks were assessed, they were then available as clues that could be used to understand any changes in flow that occurred between the control and remodeled networks. 


\section{Hemodynamic comparisons between control and remodeled networks}

\section{Flow comparisons}

The flow and pressure computations were carried out using the Hagen-Poiseuille equation. The comparison between average individual flow rates between vessels of different orders indicated that the remodeling process had led to maintaining the same average flow rates in vessels of similar caliber in the remodeled vessels as in the control (Figure 28). A comparison of the volumetric flow rates indicated that there was no significant difference in the control and remodeled networks in that aspect (Table 4).

Flow was restored to the remodeled networks from the saphenous artery (in silico) in order to quantitatively study the impact of double-sided flow entry into the gracilis artery. The total volumetric flow rates after restoration of flow from the saphenous artery were not significantly different from those of the control and remodeled networks. Since average individual flow rates and total volumetric flow rates through the remodeled networks are similar to that of the control, it can be concluded that the remodeling process following the obstruction of flow supply from the saphenous side is favorable to maintaining normal flow rates.

\section{Wall shear stress comparisons}

Along with assessing the impact of remodeling on flow, the analysis of wall shear stress (WSS) in the various orders can give insight into the effect of the feedback loop that exists between vascular remodeling and WSS. The vascular network remodels in response to alterations in the levels of WSS. Remodeling leads to change in WSS levels, which again induce remodeling, and the process continues until a stable network structure is achieved. Other stimuli 
such as circumferential wall stress and nutrient demand induce vascular remodeling in conjunction with WSS; however, in this report only WSS has been assessed.

Total removal of blood supply from the saphenous side of the microcirculation in the gracilis leads to the volumetric flow through the saphenous being rerouted through the muscular branch. There is a subsequent increase in flow through the muscular branch and the portion of the gracilis artery immediately distal to it. This leads to elevation of shear stress in parts of the gracilis artery where flow rates increase dramatically. Increase in flow leads to shear stress elevation, which triggers collateral enlargement of the gracilis. In the images of the remodeled network studied in this report, enlargement of the gracilis artery near the muscular branch was observed. Consistent with this observation is the decrease in WSS observed in the order 5 vessels which mainly consist of vessel segments of the gracilis fed by the muscular branch, since an increase in diameter leads to lowering of WSS due to the inverse dependence of WSS on the third power of diameter (Equation 1) (Figure 33).

After 14 days of remodeling the order 4 vessels have WSS levels significantly higher than that of the control due to the slightly lower average diameter of order $4 \mathrm{~s}$ in the remodeled networks (Figure 39) (Table 5). This piece of information prompts further study of the effects of remodeling after a more stable network topology is reached in future in order to observe whether or not the average diameter of the order $4 \mathrm{~s}$ increase in order to lower the WSS. Perhaps the individual average flow rates in orders 2 and 3 in the remodeled are the same as in the control because remodeling has maintained the order 4 flow rates in the remodeled near the corresponding control values. Studying the remodeling process for a greater number of days can reveal whether order $4 \mathrm{~s}$ will enlarge in diameter and if so, whether the flow rates in orders 2 and 3 will change substantially as a result. A quantitative assessment will give insight into the 
compromise made between relative increase in flow and decrease in WSS that would occur through increase in diameter.

\section{Correlations between architectural changes and hemodynamic alterations post remodeling}

Individually, the models used to describe the architecture and flow in the control and remodeled networks shed light on some significant and interesting changes. Both control and remodeled networks show adherence to Horton's Law, and the connectivity matrices indicate that substantial changes in connectivity occur between the higher order vessels. There is a significant drop in the average quantity of order 4 vessels and a slight increase of the average quantity of order 5 vessels in the remodeled networks relative to those in the control.

Additionally, the percentage of order 4-order 4 connections in the remodeled is substantially lower and the percentage of order 5-order 4 connections is substantially higher in the remodeled networks relative to those in the control. Given the same pressure boundary conditions for the control and remodeled networks, as well as similar morphology of the lower caliber vessels, it would be reasonable to expect that the average flow rates in order 4 vessels in the remodeled networks would be nearly equal to or higher than that of the control because most of their flow supply comes from order 5 vessels, and is shared by a relatively small quantity of order $4 \mathrm{~s}$. The average individual flows are consistent with this expectation as the order 4 vessels in both the control and remodeled have equal average flow rates despite the average flow rate in order $5 \mathrm{~s}$ being slightly lower than that in the control (Figure 33). On restoring flow through the saphenous artery computationally (and keeping the pressure boundary conditions the same as the control), the effect of the architectural changes in the order 4 and order 5 vessels comes through as being consistent with our expectations as well, since the average double-sided remodeled flow 
rate through the order 4 vessels increases compared to that in the control and remodeled networks (although the increase is not statistically significant) (Figure 29).

Drawbacks in current models for comparative purposes and future work Vessel ordering and hemodynamic comparisons

While some correlations could be made between the flow changes and architectural alterations, the comparisons were not highly effective and informative. All the morphological and hemodynamic characteristics were assessed with respect to order, but ordering process itself had some flaws that led to lack of effective representation of the data. There are several vessels that fall within the same branching hierarchy, but do not share similar vascular caliber and the diameter-defined Strahler method attempts to capture the degree of diameter variation occurring at each branching level. However, this method fails to reflect the correct branching hierarchy of the vessels in an attempt to capture the heterogeneity in vascular caliber among vessels belonging to the same branching level (Figure 39).

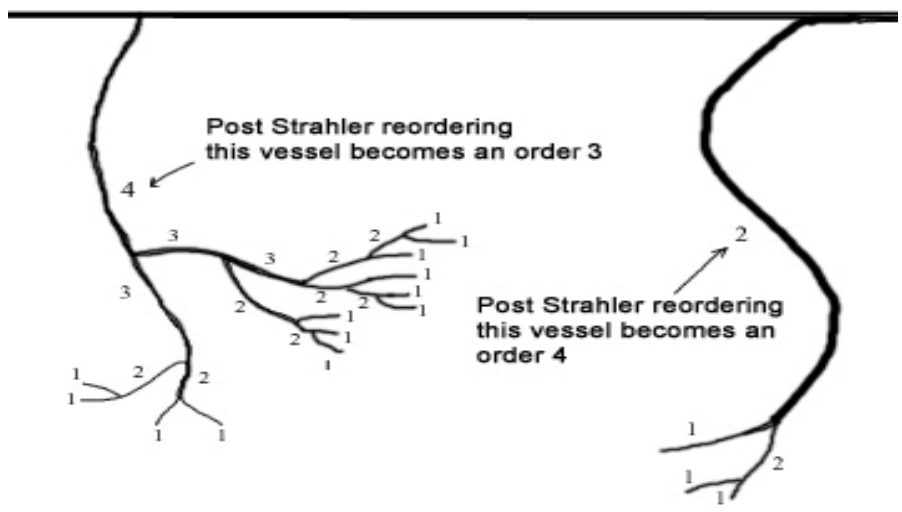

Figure 39. Inadequate reflection of branching hierarchy of vessels through diameter based reordering in diameter-defined Strahler method. 
In order to analyze the architectural characteristics and flow variations in the network it will perhaps be useful to order the vessels as per the original Strahler ordering scheme and not conduct any reordering based on diameters of vessels. Also, another separate ordering scheme may be applied in which orders can be based purely on arbitrary diameter bounds alone. In this manner two separate analyses can be used to study how network properties change. For instance, an assessment can be made regarding the variation in pressure with respect to decreasing branching hierarchy in the network. Another study can be done regarding the flow variation across the network based purely on vessel diameter trends. Applying these types of ordering schemes will also eliminate the need for applying the diameter bounds of various orders in one network on the other. The unique branching characteristics of each network can be assessed, and the heterogeneity in flow and pressure for each branching level can be compared for the normal and remodeled networks.

In addition to performing ordering of vessels based on one particular defining characteristic such as branching hierarchy for comparative analysis, the variation of morphological and hemodynamic characteristics from one side of the gracilis (fed by muscular branch) to the other (fed by saphenous branch) can also be assessed. This can reveal interesting patterns on the variation of diameter, quantity, lengths, as well as flows in gracilis and transverse arteriole segments from one section of the muscle to the other. Poorly perfused regions of the muscle post arterial occlusion can be revealed through such studies. This information can be useful in determining parts of the microcirculation where further collateral enlargement or angiogenesis can restore flow.

Finding regions with inadequate perfusion and introducing changes in connectivity of vessels in those areas or altering the vessel diameter distributions can reveal the impact of these 
morphological changes on flow rates. Even with the current analysis it will be interesting to study how individually altering morphological features such as connectivity, diameter distributions and length distributions of vessels in different orders affects the network flow. Only when the effect of individual morphological parameters are teased apart in comparative studies between networks, can more confident analysis be made concerning the correlations between architectural changes and flow.

Finally, in future studies it will be useful to determine the actual pressure conditions in the capillaries and the feed arteries in order to more accurately model the flow and pressures post remodeling. Additionally, viscosity gradients throughout the network can be established to model network hemodynamics more effectively.

\section{Improvements in morphological analysis}

As mentioned previously in this section of the report, it was difficult to capture the small vessels $(\sim 5-8 \mu \mathrm{m})$ due to the limitations in image resolution as well as the movements of the vessels perpendicular to the plane of the image. Even the slight movement of a small diameter vessel in the z-direction led to a large error in the measurement of its length and diameter. In future the use of imaging techniques such as confocal microscopy to obtain 3D representations of the vasculature can help the researcher to trace the movements of the vessels in the $\mathrm{z}$-direction and obtain more accurate length and diameter measurements of vessels. 


\section{Chapter Three \\ Introduction \\ Types of theoretical models in microcirculation research}

Theoretical models such as those described in the previous chapter are used for the purpose of understanding the structure and dynamics of the microcirculation. Other types of models study the molecular and cellular mechanisms involved in microcirculatory function, and still others look at the relations between microcirculatory structure and dynamics and molecular and cellular mechanisms. Each model is designed and developed based on the scope and objective of the study.

Several different types of models have been created and used over the years in microcirculation research. More recently an effort has been made to categorize and organize the information on these models in order to put previous research into perspective, as well as make it useful in providing direction for future studies. Following are some categories of models that are useful in defining the various types of theoretical models that have been created thus far in microcirculatory research:

Phenomenological models: In phenomenological models observed properties are described using fitted equations or other mathematical relationships that are not based on the mechanisms of the underlying processes. These models help to create a mathematically compact representation of a complex set of data and can be used in the development of higher level models. Phenomenological models cannot be extrapolated to predict behavior outside of the underlying experimental data.

Qualitative conceptual models: A qualitative conceptual model is based on a hypothesis about the mechanism underlying an observed phenomenon. The mechanism is expressed in 
mathematical terms, and the range of possible predicted behaviors is compared with the observed behavior. Model parameters are not necessarily evaluated in such models.

Quantitative conceptual models: Similar to qualitative conceptual models, these models are based on hypotheses regarding mechanisms underlying system behavior. However, in this case, the theoretical descriptions of the mechanisms are fully parameterized. Some parameter values are known beforehand, and the question being addressed is whether model predictions are quantitatively consistent with observed behavior. If this is not possible for suitable values of unknown parameters, then the hypothesized mechanisms are either rejected or deemed to be inadequately represented in the model. However, if model results are consistent with observed behavior, then the unknown parameters are estimated through a process of optimization for best agreement between prediction and experimental data.

Predictive (application) models: Such models are constructed when the underlying mechanisms are known with a reasonable level of confidence. The model is constructed to predict system behavior and results are compared quantitatively with observations of system behavior, without the need for parameter fitting or optimization. If significant disagreements are found, the original assumptions are inferred incorrect or inadequate, and the model reverts to the category of quantitative conceptual models (Secomb, Beard et al. 2008).

\section{Parameter sensitivity analysis}

In chapter two it was found that the previous descriptive models used to compare the topology and flow properties of normal and remodeled vascular networks did not provide sufficient detail to correlate architectural alterations that occur during remodeling to changes in flow properties. Significant differences in architecture between the control and remodeled networks were discovered, however, it was difficult to directly correlate the morphological parameter variations to flow properties. 
For the purpose of effective analysis of remodeling in networks in future studies, a parameter sensitivity analysis tool (PSAT) was created. The PSAT was developed so that the investigator could alter individual morphological parameters and quantitatively study their impact on flow. The connectivity of vessels within the same order as well as among different orders can be altered arbitrarily using the PSAT. Additionally, the distribution of vessel diameters and vessel lengths within and across the various orders can be altered. Flow computations following these morphological changes can then reveal the quantitative role of these parameter alterations on flow changes.

Performing parameter sensitivity analysis has an additional advantage apart from assessing flow sensitivity to various architectural parameters. The tool allows the researcher to perform in silico changes to network architecture that cannot be done experimentally. In previous microcirculation research theoretical models have been created that allow researchers to perform in silico experiments that cannot be done experimentally in order to gain further insight into microcirculatory phenomena (Skalak, Price et al. 1998; Pries and Secomb 2000; Bailey, Thorne et al. 2007). The insights gained from the results of such in silico experiments can be used to create better hypotheses for future experimental studies.

\section{Predictive versus descriptive models}

Models such as the PSAT allow investigators to run theoretical experiments on systems and observe the system's response. However, it is important to note at this point that the goal of models such as the PSAT is not to predict system behavior using a model based on a mechanistic understanding of the system. Thus, the PSAT is not a predictive model. Validation is an indispensable step in predictive models. The attempted predictive response to an applied stimulus must be compared to experimental results to ensure that the general assumptions and simplifications made in the model are reasonable for prediction purposes. In descriptive models, 
comparison with experimental results for validation is not necessary, as the goal is to use a mathematical tool to study measurable differences before and after the application of a stimulus using data from previous experiment(s). Our model has 2 components: quantitative description of network topology and dimensions and flow characteristics of the network. The first component (including Horton's law, Strahler ordering) does not require validation. The trends followed by the diameters, lengths, and quantities of vessels along different orders can be checked to determine whether they are consistent with Horton's law. Similarly, the diameterdefined Strahler ordering scheme can be applied on the data and it can be checked whether overlap of diameters among orders is preventable using this ordering scheme. However, the second component for hemodynamic analysis requires validation by comparison to experimental values of flow (Figure 31, 32).

The PSAT alters network topology as according to the user's needs. The resultant effect on flow is used to determine the quantitative impact of topological change on flow. The PSAT however, does not use any previously determined principles of vascular remodeling in order to change the network structure and dimensions, and thus, does not require validation of any principles, assumptions, or simplifications from experimental studies.

\section{Automation of Strahler ordering and shifting software platforms}

While performing the current study it was found that the process of morphological analysis and flow computations is fairly time consuming and repetitive when several muscle samples are analyzed. Therefore, it was considered worthwhile to work on automating the morphological analysis and making the process of flow computations more user-friendly. The progress made on this front is discussed in this report. 
Ordering 300-500 vessels based on the Strahler method was a fairly time consuming process, especially when 10 muscle samples were being analyzed. Additionally, the probability of making errors while ordering was also very high. Thus, an algorithm was created that would order all the vessels identified in a muscle sample given the individual vessel labels and their corresponding parent labels.

In addition to automating the ordering process, the software used for flow and pressure calculations was changed from Mathematica to MATLAB. This was done so that the number of steps involved in inputting equations into the software to generating flow rates and pressures for the various orders could be done in fewer steps. Reducing even a few steps makes a great difference with respect to time and effort involved when the procedure has to be repeated for a large number of samples. Additionally, MATLAB is more conveniently accessible to department members in the university and offers easier ways of identifying errors in the code and troubleshooting compared to Mathematica. The PSAT has also been created in MATLAB. The ability to extract information from Excel files and write information into them without manual interventions, made the use of MATLAB convenient for the design of the PSAT. 


\section{Methods \\ Overview}

The main objective was to design a mathematical tool that would allow the testing of flow rate sensitivity to morphological parameters throughout the various orders of vessels in the network. Also, an algorithm for ordering vessels in a network based on the Strahler ordering scheme was to be created for automation of the morphological analysis procedure, and flow and pressure calculations were to be shifted to MATLAB from Mathematica.

The PSAT is mainly composed of three component MATLAB programs, each of which allows alteration of vascular connectivity, diameter distribution, and length distribution for any network under study. Some computations, as well as organization of vessel information is done in Excel before the data is sent to MATLAB for the actual alteration of morphological information. The most challenging algorithm was the one that would allow the user to arbitrarily alter the connectivity of vessels of various orders without altering other parameters such as diameters or lengths. All the codes required the vessel segments to retain all the information concerning their structure and topology except the particular parameter being altered. Additionally, all the codes allow the user to read information concerning the connectivity, or diameter, or length distributions from one network and impose those morphological characteristics on another network. In the current study, the PSAT has been used to impose the connectivity characteristics of the control network on the corresponding remodeled network, while arbitrary changes were made to the diameter and length distributions. The impact of these changes on flow throughout the networks was then assessed. 


\section{Parameter Sensitivity Analysis Tool}

\section{Connectivity alteration algorithm}

In order to change the connections between vessels of different orders without changing the diameter and length characteristics of the vascular network, the idea developed was to "break apart" or disassemble the inherent vessel connections within the network being analyzed. In this way each vessel segment would retain its complete individuality, and the only information that would be lost is its connection with other vessels (Figure 40).

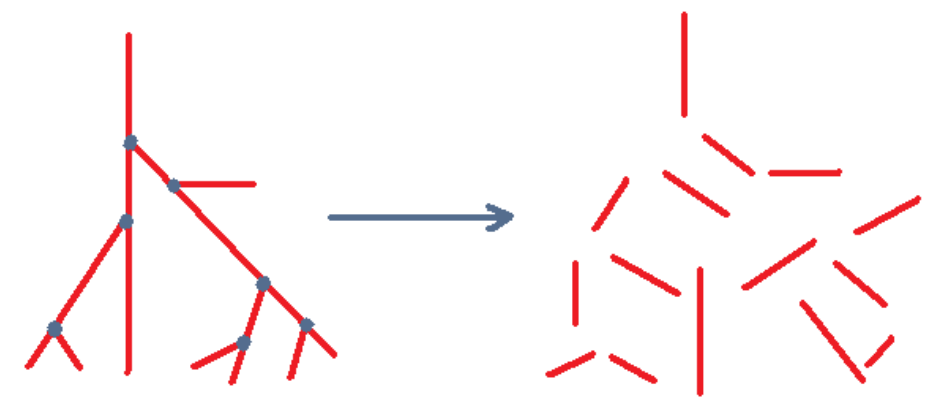

Figure 40. Removal of vessel connectivity information from the original network

The new ratios for the connections between vessels of different orders would then be used to connect the vessel segments and create a network which would have all its morphological characteristics similar to that of the original, except for the connections between vessels. For instance, while imposing the connectivity characteristics of the control network on the remodeled network, the parent-daughter information concerning the remodeled segments could be removed from the segmental data, while other information such as vessel labels, diameters, lengths and orders could be retained. The code would then receive the connectivity information of the control, and the vessel segment information of the remodeled network. The 
code would then connect the vessels in such a fashion that the resulting network would reflect the connectivity characteristics of the control network (Figure 41).

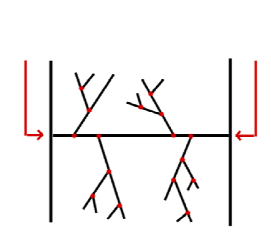

Pre-surgery normal vascular network
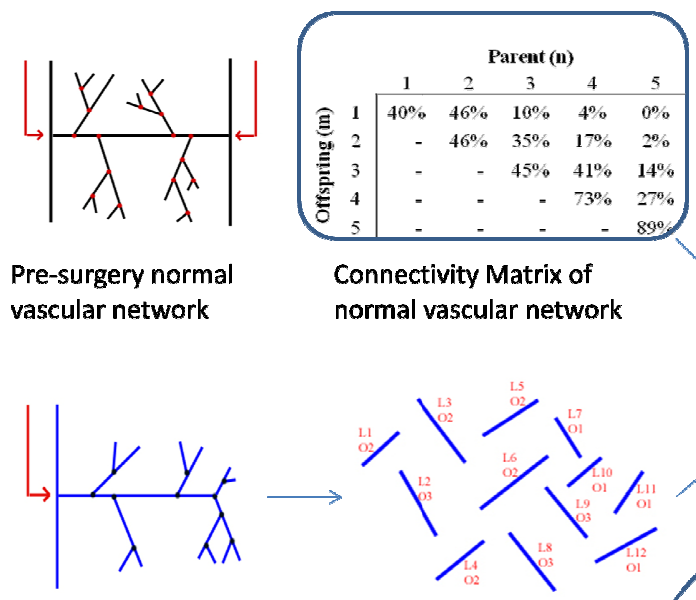

$$
\begin{aligned}
& \text { Post-surgery } \\
& \text { remodeled vascular } \\
& \text { network }
\end{aligned}
$$
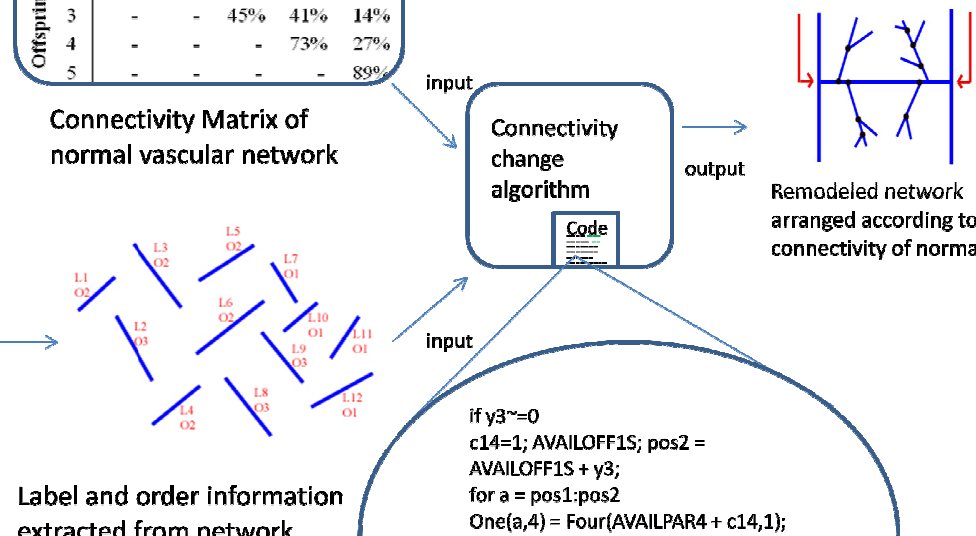

Remodeled network arranged according to connectivity of normal extracted from network

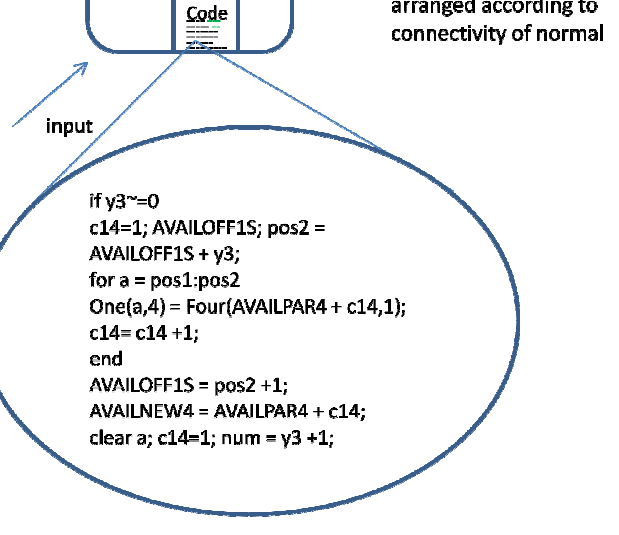

Figure 41. Pictorial representation of connectivity alteration in a remodeled network based on control connectivity ratios for various orders.

The code would also allow the user to arbitrarily change the connectivity of any network under analysis using any desired connectivity ratios.

The connectivity matrix used previously to provide information on branching characteristics of a network, was a very useful starting point in beginning to think about the logic of the code. However, hidden within this matrix was information on specific types of branches which had to be teased apart from the original data set post-reordering. For example, $34 \%$ order 1s may be springing out of order $2 \mathrm{~s}$, but the proportion of these that have sibling order $1 \mathrm{~s}$ or sibling order $2 \mathrm{~s}$ is unknown. 
This information could have been obtained from the connectivity matrix alone, if there were no deviations from the branch types observed post ordering based simply on connectivity. However, the reordering performed using the diameter-defined Strahler method gives rise to myriad branching patterns which require probing into the data to search and count every possible branch type that could occur. For this purpose, the various possible branch types that were present in the control network were determined, and their respective ratios were computed in Excel. The following figure shows the different branch types that were present in the 5 samples of control networks that were studied in this report (Figure 42):

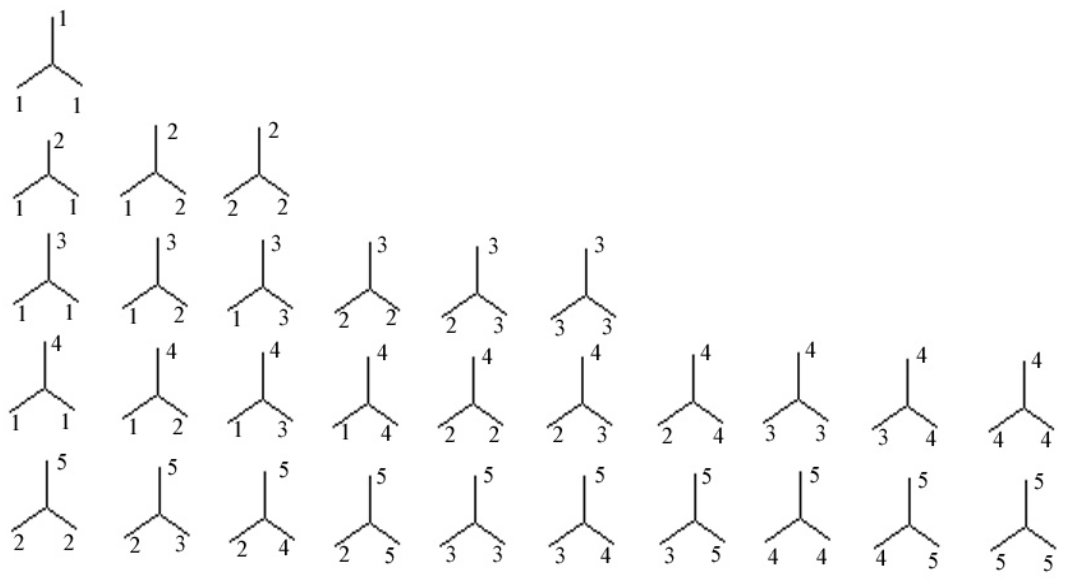

Figure 42. A schematic depicting all the different types of bifurcations found post-reordering in the control vascular networks.

Using the ratios for the various branch types in the control network, the investigator can determine the number of those respective branch types that the remodeled network will have after connectivity changes are made. It is optional for the investigator to use this component of 
the PSAT, and maintain similar ratios of individual bifurcation types in the both the network being modeled and the network being altered. Sometimes it is not even possible to maintain similar ratios due to wide variations between the numbers of vessels available in each order of the network under change. The lack of availability of vessels of certain orders then makes it difficult to impose the ratios from network to the other. However, when a large number of segments are available, performing such thorough sensitivity analysis (that is, observing how flows change when the ratios of each particular branch type in the two networks that are being compared is the same) is feasible, as there is greater flexibility in assembling the various branch types using segments from the network being altered. Additionally, leaving out a few segments (especially in the lower orders) that could not be included in order to maintain the specific bifurcation ratios, does not have a significant impact since those vessels constitute a very small percentage of the total number available vessels. When working with a small number of total vessels available in the network being altered, the investigator has to be more careful about leaving out a few segments as a compromise for maintaining bifurcation ratios, because doing so can affect the average quantity of vessels in the orders substantially.

Finally, for the sake of repeatability of experiments, it was important that for the same input information regarding network connectivity and vessel segments, the code would assemble the segments in exactly the same fashion (i.e, each vessel would be connected to the same parent and would have the same vessels as offspring).

An algorithm was developed that met the criteria set forth for the connectivity alteration component of the PSAT. It was determined that the code would require vessel label and order information as inputs, and while the program was running, the number of offspring and parent label assigned to each segment would need to be continually updated. Given the type of input 
data and the computational work to be performed by the code, it was decided that it would be best if the code could be written using software in which working with matrices was convenient. MATLAB was selected as a suitable software platform for creating the code, primarily because it is a high-level computing language that allows convenient matrix manipulation with control flow statements and functions. It also provides an interactive environment for algorithm development and management of code and data files.

\section{MATLAB features used in PSAT development}

In MATLAB, it is possible to enter one command at a time at the MATLAB command line, or to write a series of commands to a file that can be executed as an entire MATLAB program. The MATLAB editor can be used to create program files. The two types of program files in MATLAB are scripts and functions. Scripts do not accept input arguments or return output arguments. They operate on data in the workspace. The workspace consists of the set of variables created during a MATLAB session. Functions are the second type of program files that can accept input arguments and return output arguments. Their internal variables are local to the function. The PSAT consists of several script files and Excel files. When script files are run, data from the Excel files is read and post manipulation, the output is written into specific sheets in the Excel files. This eliminates the need to copy and paste data from several Excel sheets and thus, saves time and reduces chances of error.

The function used for conditional program control in the PSAT code is the "if...else" statement that evaluates a logical condition and executes following the "if" command if the expression evaluated is true. Otherwise, the statements following "else" are executed. In order to repeat a set of commands a predetermined number of times the "for loop" was used. Using the "for loop" one can define the number of times a set of statements will be executed. Another loop used was the "while loop" that repeats a group of statements indefinite number of times under 
the control of a logical condition. Further explanation of specific statements used in the code is provided in the appendices.

\section{Diameter and length alterations through PSAT}

The two other components of the PSAT apart from the connectivity alteration tool are the ones that allow diameter and length distribution changes. Each vessel order has a range of vessel diameters and lengths. The algorithms for these two components of the code were fairly similar. The distribution of diameters and lengths is measured in Excel using the vessel data postreordering. Within a given range of diameters for each order, an Excel formula counts the number of segments at each tenth of a micron. A percent frequency distribution is obtained which is used by the MATLAB code to alter the distribution of diameters in another network according to the measured distribution (Figure 43). It is important to impose the diameter bounds of the reference network on the network being altered prior to running this code to avoid situations where the diameter of a feeding vessel is larger than the diameter of the offspring vessel. Since the lengths of vessels within an order vary drastically, counting vessels at each tenth of a micron or even a micron is not practical. Therefore, determining the frequency distribution has been left to user discretion. For instance, the user can count the number of vessels falling within a certain range and decide to assign the average length in this range to the corresponding ratio of same order vessels in the network undergoing change (as has been done in this study). Other approaches may also be used as long as the percent frequencies and length values to be assigned are determined. 


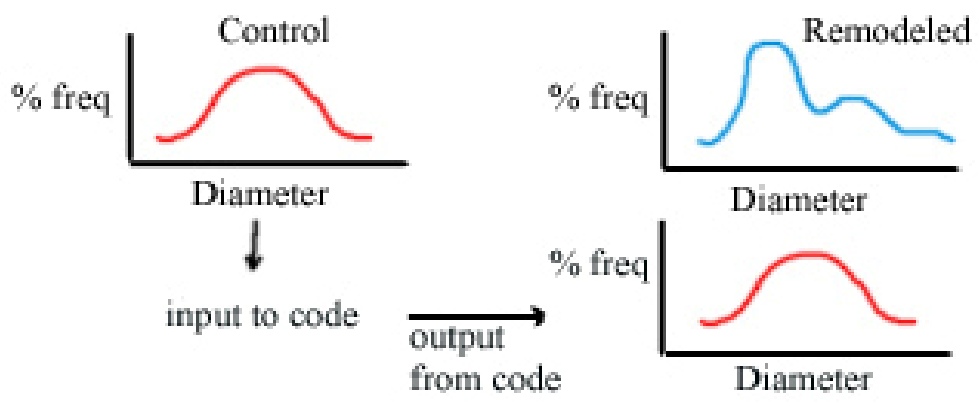

Figure 43. Schematic showing the imposition of the diameter distribution in an order of a control network on that of the same order in the remodeled network.

\section{Automation of Strahler vessel ordering}

As described previously, the Strahler ordering process assigns the lowest order (1 was selected for the current study) to the terminal vessel segments in a vascular tree. When two vessels of order $n$ meet, the parent vessel is assigned an order $n+1$. When two vessels of unlike orders $n$ and $m$ meet, the parent vessel retains the higher of the two daughter orders.

The user is only required to enter the information regarding the vessel segment labels and the corresponding parent segment labels for this code. In Excel, the terminal vessels are identified and are assigned order 1 . Following this, the MATLAB code reads the three columns of information, namely, segment labels, parent labels, and segment orders from the Excel sheets. The Strahler ordering principle is then used to assign orders to parent segments. A loop is run to keep assigning order numbers to vessels until there are no vessels left without an assigned order.

\section{Shifting software platforms from Mathematica to MATLAB}

Since the PSAT was developed using Excel and MATLAB, and the steps involved in the flow calculations could be made less time consuming using MATLAB, the entire software platform for programming and computations was set up in MATLAB. Previously our lab was using Mathematica for flow computations. However, due to the reasons just mentioned and the 
more convenient accessibility of the MATLAB software to the department members, the shift to MATLAB was made. The setup and organization of equations, constants, and variables is now performed in Excel for input to MATLAB. The user still has a choice to generate equations in a format that could be used for solving in Mathematica by making a few minor changes. The format for entering equations to be solved by MATLAB is slightly different than that in Mathematica. A portion of the flow calculations code in MATLAB is shown below to illustrate how equations are listed and solved (more details are in the appendix):

syms P8 P9 P10 P11 P12 P13 q8 q9 q10 q11 q12 q13

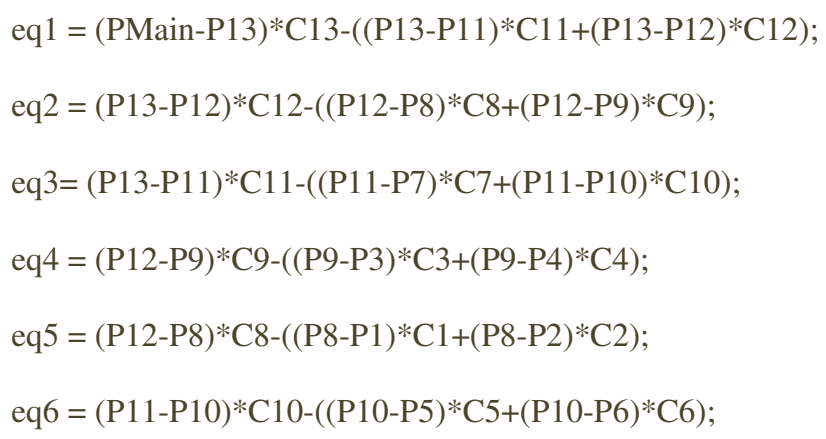

\section{Statistical analyses}

Student's t-test was used to make comparisons between remodeled and altered remodeled network morphology and hemodynamics. 
Results

\section{Connectivity change}

In order to demonstrate how the PSAT can be used for altering connectivity between two networks, as well as to determine how the flows change when the connectivity of the control networks is imposed on the remodeled, connectivity alteration component was used to impose the control connectivity of all the 5 control networks on their corresponding remodeled networks.

Connectivity for the higher orders was very difficult to impose given the really small number of higher order vessels. However, the connectivity ratios for the higher orders used for the alteration were not found to be statistically different from those of the control. The connectivity ratios for the smaller orders could be imposed very closely as shown in Figure 44.

\begin{tabular}{|c|c|c|c|c|c|c|}
\hline \multirow{2}{*}{\multicolumn{2}{|c|}{ Connectivity change average $\%$}} & \multicolumn{5}{|c|}{ Parent } \\
\hline & & 1 & 2 & 3 & 4 & 5 \\
\hline \multirow{5}{*}{ 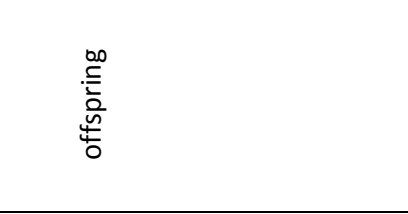 } & 1 & $33 \%$ & $52 \%$ & $13 \%$ & $2 \%$ & $0 \%$ \\
\hline & 2 & $0 \%$ & $46 \%$ & $43 \%$ & $10 \%$ & $2 \%$ \\
\hline & 3 & $0 \%$ & $0 \%$ & $51 \%$ & $38 \%$ & $11 \%$ \\
\hline & 4 & $0 \%$ & $0 \%$ & $0 \%$ & $51 \%$ & $49 \%$ \\
\hline & 5 & $0 \%$ & $0 \%$ & $0 \%$ & $0 \%$ & $92 \%$ \\
\hline \multicolumn{2}{|l|}{ Control average $\%$} & 1 & 2 & 3 & 4 & 5 \\
\hline \multirow{5}{*}{ 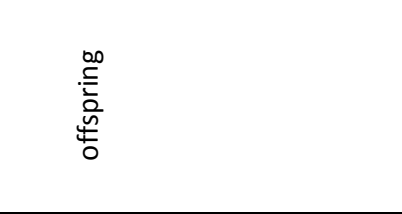 } & 1 & $33 \%$ & $53 \%$ & $13 \%$ & $2 \%$ & $0 \%$ \\
\hline & 2 & $0 \%$ & $45 \%$ & $43 \%$ & $11 \%$ & $1 \%$ \\
\hline & 3 & $0 \%$ & $0 \%$ & $51 \%$ & $43 \%$ & $5 \%$ \\
\hline & 4 & $0 \%$ & $0 \%$ & $0 \%$ & $64 \%$ & $31 \%$ \\
\hline & 5 & $0 \%$ & $0 \%$ & $0 \%$ & $0 \%$ & $79 \%$ \\
\hline \multirow[t]{2}{*}{ Remodeled average \% } & & 1 & 2 & 3 & 4 & 5 \\
\hline & 1 & $33 \%$ & $49 \%$ & $15 \%$ & $4 \%$ & $0 \%$ \\
\hline \multirow{4}{*}{ 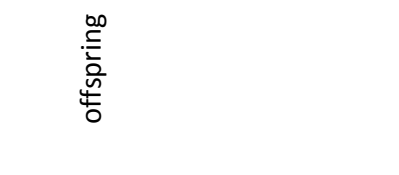 } & 2 & $0 \%$ & $42 \%$ & $46 \%$ & $9 \%$ & $3 \%$ \\
\hline & 3 & $0 \%$ & $0 \%$ & $54 \%$ & $31 \%$ & $15 \%$ \\
\hline & 4 & $0 \%$ & $0 \%$ & $0 \%$ & $44 \%$ & $55 \%$ \\
\hline & 5 & $0 \%$ & $0 \%$ & $0 \%$ & $3 \%$ & $89 \%$ \\
\hline
\end{tabular}

Figure 44. Comparison of average connectivity percentages for control, remodeled, and remodeled networks with altered connectivity. 


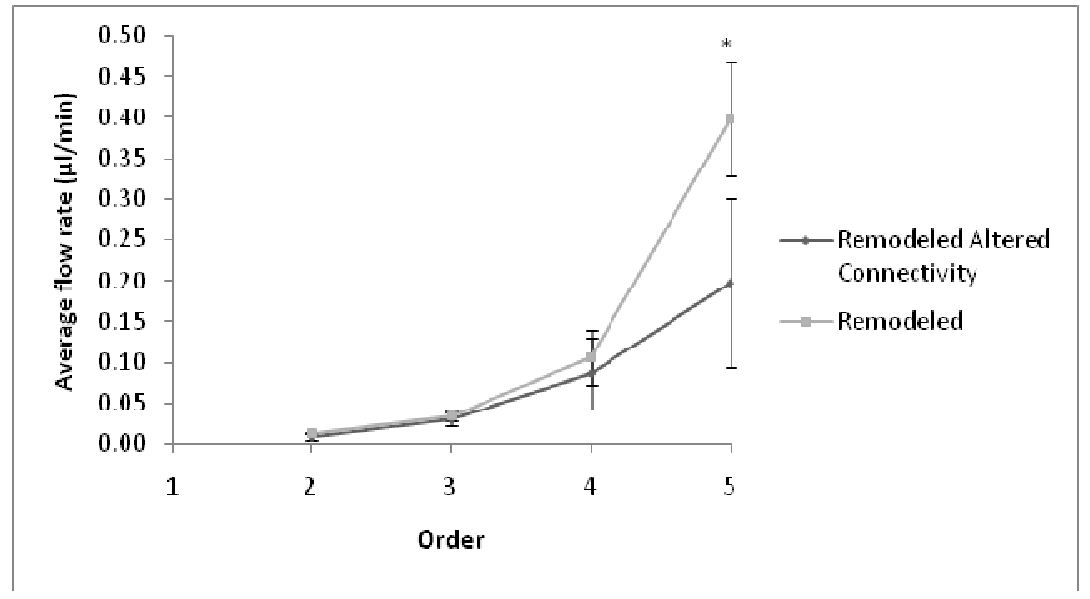

Figure 45. Comparison of average individual flow rates for all the orders between the remodeled networks and remodeled networks with altered connectivity.

On average, the individual vessel flow rates for order 5 vessels in the drops sharply after connectivity changes in the remodeled networks. Even though there is no statistical difference between the connectivity ratios in the altered remodeled network and the control network, it is interesting that changing the lower order connectivity did not bring about a significant impact in the individual flow rates, whereas changing the higher order connections did.

The total volumetric flow rates in the networks with altered connectivity were much lower than that of either the control or the remodeled (Table 7).

Table 7. Comparison of total volumetric flow rates in the altered remodeled network with those of the control and original remodeled networks.

\begin{tabular}{c|c|c|c|c|c|c|c|c}
\hline Control & $\begin{array}{c}\text { Flow rate } \\
(\mathrm{ml} / \mathrm{min} / \mathrm{g})\end{array}$ & SE & Remodeled & $\begin{array}{c}\text { Flow rate } \\
(\mathrm{ml} / \mathrm{min} / \mathrm{g})\end{array}$ & $\mathrm{SE}$ & $\begin{array}{c}\text { Altered } \\
\text { remodeled }\end{array}$ & $\begin{array}{c}\text { Flow rate } \\
(\mathrm{ml} / \mathrm{min} / \mathrm{g})\end{array}$ & SE \\
\hline M1 & 0.19 & 0.14 & M1 & 0.13 & 0.07 & M1 & 0.04 & 0.04 \\
M5 & 0.39 & 0.1 & M5 & 0.24 & 0.09 & M5 & 0.19 & 0.04 \\
M6 & 0.27 & 0.08 & M6 & 0.2 & 0.08 & M6 & 0.19 & 0.05 \\
M7 & 0.2 & 0.06 & M7 & 0.21 & 0.07 & M7 & 0.10 & 0.03 \\
M8 & 0.22 & 0.08 & M8 & 0.23 & 0.1 & M8 & 0.10 & 0.02 \\
Average & $\mathbf{0 . 2 5}$ & $\mathbf{0 . 1 4}$ & Average & $\mathbf{0 . 2}$ & $\mathbf{0 . 1}$ & Average & $\mathbf{0 . 1 2}$ & $\mathbf{0 . 0 5}$
\end{tabular}




\section{Diameter distribution change}

The diameter distributions of orders 4 and 5 were changed in the remodeled network to match those of the control, and the differences in flow were assessed.

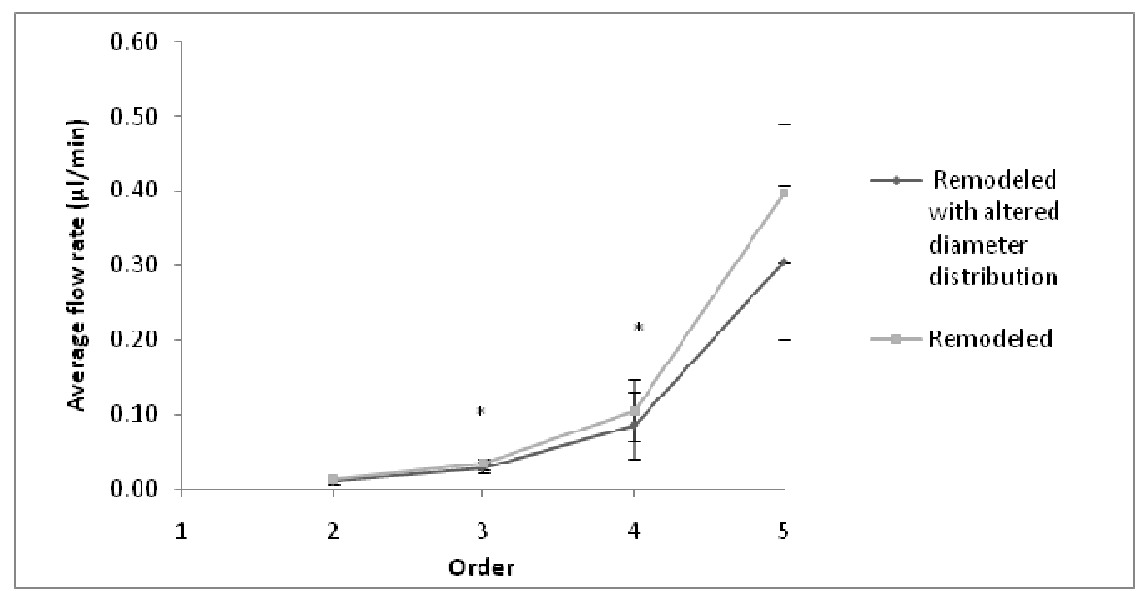

Figure 46. Comparison of average individual flow rates for all the orders between the remodeled networks and remodeled networks with altered diameter distribution.

Imposing the order 4 and 5 diameter distributions of the control on the remodeled led to a drop in the average order 4 and 5 flows. However, statistical differences were observed between the flow rates of order $3 \mathrm{~s}$ and order $4 \mathrm{~s}$. The statistical difference in the flows for order $3 \mathrm{~s}$ might be because of the wide variation in order 3 flows due to the diameter alterations in order $4 \mathrm{~s}$ and 5s. However, the mean order 3 flow rates turned out to be identical in both the altered and original remodeled networks. 
Table 8. Comparison of total volumetric flow rates in the altered remodeled network with those of the control and original remodeled networks.

\begin{tabular}{c|c|c|c|c|c|c|c|c}
\hline Control & $\begin{array}{c}\text { Flow rate } \\
(\mathrm{ml} / \mathrm{min} / \mathrm{g})\end{array}$ & SE & Remodeled & $\begin{array}{c}\text { Flow rate } \\
(\mathrm{ml} / \mathrm{min} / \mathrm{g})\end{array}$ & $\mathrm{SE}$ & $\begin{array}{c}\text { Altered } \\
\text { remodeled }\end{array}$ & $\begin{array}{c}\text { Flow rate } \\
(\mathrm{ml} / \mathrm{min} / \mathrm{g})\end{array}$ & SE \\
\hline M1 & 0.19 & 0.14 & M1 & 0.13 & 0.07 & M1 & 0.07 & 0.02 \\
M5 & 0.39 & 0.1 & M5 & 0.24 & 0.09 & M5 & 0.23 & 0.02 \\
M6 & 0.27 & 0.08 & M6 & 0.2 & 0.08 & M6 & 0.21 & 0.01 \\
M7 & 0.2 & 0.06 & M7 & 0.21 & 0.07 & M7 & 0.11 & 0.01 \\
M8 & 0.22 & 0.08 & M8 & 0.23 & 0.1 & M8 & 0.18 & 0.02 \\
Average & $\mathbf{0 . 2 5}$ & $\mathbf{0 . 1 4}$ & Average & $\mathbf{0 . 2}$ & $\mathbf{0 . 1}$ & Average & $\mathbf{0 . 1 6}$ & $\mathbf{0 . 0 2}$
\end{tabular}

As expected, the total volumetric flow rates through the networks drop when the diameter distributions of the larger caliber vessels in the remodeled network are changed to match those of the control. There is a $20 \%$ and a $16 \%$ drop in total volumetric flow in the altered remodeled network compared to the control and the original remodeled networks, respectively.

\section{Length distribution change}

The diameter distributions of orders 2,3 , and 5 were changed in the altered network, and the flow results were compared prior to and post alterations.

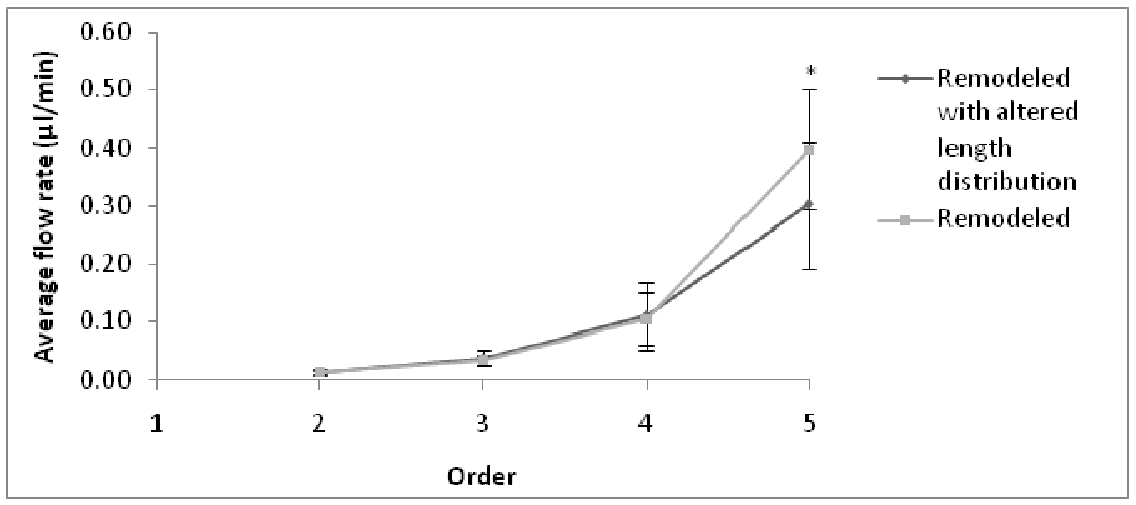

Figure 47. Comparison of average individual flow rates for all the orders between the remodeled networks and remodeled networks with altered length distribution. 
After changing the length distributions in the remodeled network for orders 2, 3, and 5, a significant change in flow was observed only in order 5 vessels. Changing the length distributions in the lower orders did not impact the flow rates.

The total volumetric flow rates in all the altered remodeled networks decreased upon altering the length distributions (Table 9).

Table 9. Comparison of total volumetric flow rates in the altered remodeled network with those of the control and original remodeled networks.

\begin{tabular}{c|c|c|c|c|c|c|c|c}
\hline Control & $\begin{array}{c}\text { Flow rate } \\
(\mathrm{ml} / \mathrm{min} / \mathrm{g})\end{array}$ & SE & Remodeled & $\begin{array}{c}\text { Flow rate } \\
(\mathrm{ml} / \mathrm{min} / \mathrm{g})\end{array}$ & SE & $\begin{array}{c}\text { Altered } \\
\text { remodeled }\end{array}$ & $\begin{array}{c}\text { Flow rate } \\
(\mathrm{ml} / \mathrm{min} / \mathrm{g})\end{array}$ & SE \\
\hline M1 & 0.19 & 0.14 & M1 & 0.13 & 0.07 & M1 & 0.13 & 0.04 \\
M5 & 0.39 & 0.1 & M5 & 0.24 & 0.09 & M5 & 0.22 & 0.07 \\
M6 & 0.27 & 0.08 & M6 & 0.2 & 0.08 & M6 & 0.29 & 0.08 \\
M7 & 0.2 & 0.06 & M7 & 0.21 & 0.07 & M7 & 0.11 & 0.03 \\
M8 & 0.22 & 0.08 & M8 & 0.23 & 0.1 & M8 & 0.18 & 0.05 \\
Average & $\mathbf{0 . 2 5}$ & $\mathbf{0 . 1 4}$ & Average & $\mathbf{0 . 2}$ & $\mathbf{0 . 1}$ & Average & $\mathbf{0 . 1 9}$ & $\mathbf{0 . 0 8}$
\end{tabular}




\section{Discussion \\ Recap and discussion of results}

Using the PSAT it was found that imposing user-defined connectivity, diameter, and length characteristics on the remodeled led to changes in both the average individual flow rates of the higher orders and the total volumetric flow rates for all the muscle samples. Alterations to the architectural characteristics of the lower order vessels (1, 2, and 3) did not affect the average individual flow rates in the altered remodeled networks.

From the results of this entire study it is apparent that the remodeling that occurs in the gracilis microcirculation post arterial occlusion leads to maintenance of individual vessel flow rates and overall flow rates at the normal levels.

An interesting and important addition to the PSAT will be a component that will allow alterations to quantity of vessels of various orders without affecting other parameters such as vessel connectivity, diameter distributions, as well as length distributions. Also, it will be very useful if the alterations brought about in the remodeled networks (or any other arbitrary network undergoing change) can be represented pictorially. Perhaps one way to go about doing this is to write the code to generate a plot in MATLAB that represents a bifurcation. Every subsequent bifurcation can then be created using a similar code that can be added to the existing bifurcation(s) by joining one end of the daughter vessel in the new bifurcation to the parentvessel in the previous bifurcation.

\section{Further improvements in numerical model}

The current model for the assessment of vascular hemodynamics uses the HagenPoiseuille relationship between pressure and flow to compute the individual flow rates of the vessel segments and the pressures at their inlets. However, a more accurate way of solving for the flows and pressures is to compute a Navier-Stokes solution using software that allows 
coupled FEA-CFD analysis. In such an analysis the geometry of the vessel is taken into account more accurately. For instance, the effects of branch angles and tortuosity of blood vessels are included which were not assessed in the current study. Tortuosity of blood vessels was not widely observed in the current muscle samples. However, in one of the remodeled vessel networks, considerable tortuosity was observed in a portion of the gracilis artery (Figure 48). If tortuosity is frequently observed in a larger number of samples in future studies, including its effects in the analysis of vascular remodeling will be warranted.

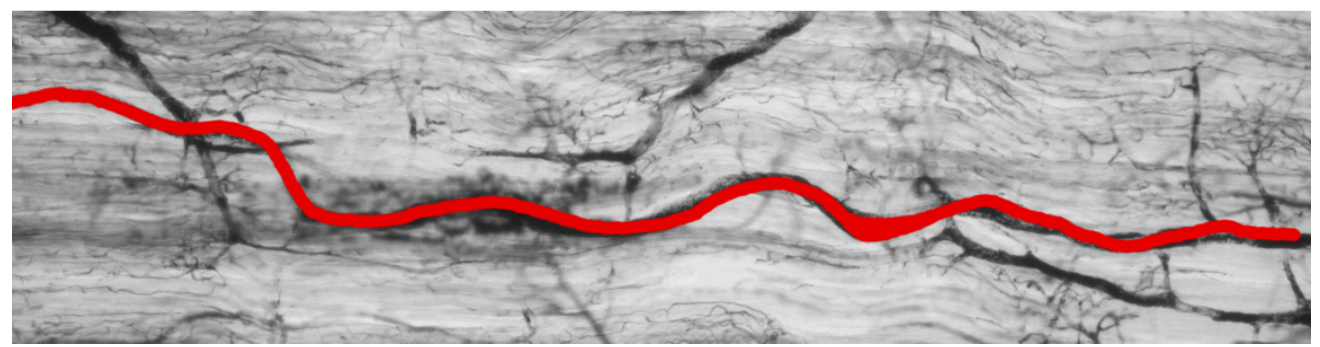

Figure 48. Portion of a tortuous remodeled gracilis artery.

COMSOL Multiphysics software can be very useful in modeling flow through the vascular networks (Figure 49). Setting up the model is convenient and quick in COMSOL since there is a physics interface available for applications in fluid flow. It is also easy to define properties about blood and the vessel wall, set boundary conditions, and solve for flows rates within the vasculature. The current challenge is to determine a way to convert the vascular image files into file types that COMSOL can both read and write. In future studies, the use of COMSOL to study microvascular flow can definitely give greater insights into vascular remodeling and its hemodynamic consequences. 


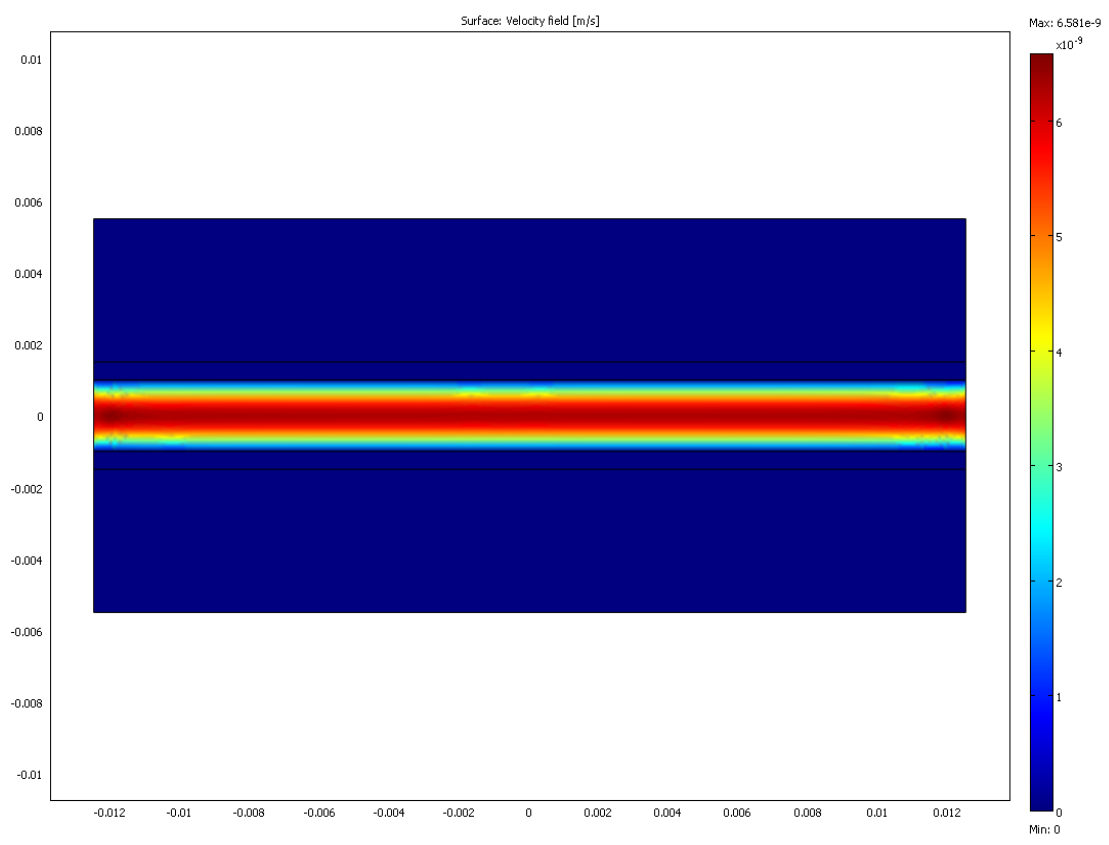

Figure 49. Velocity field in the model of a normal blood vessel created using COMSOL.

Significant improvements in future studies can also be brought about by automating the process of morphological analysis. The identification of arteriolar vessels, labeling of individual vessel segments, and measurements of their structural dimensions is a fairly time consuming task, especially when several vascular network samples are being analyzed. Manually measuring and recording the anatomical features of the vascular network is also highly prone to human error. In previous research involving the study of retinal microvascular networks, automation procedures for the morphological analysis of the networks were created (Can, Shen et al. 1999). Such automation procedures, however, cannot be applied to the mouse gracilis muscle because of the lack of visibility of small caliber vessels and substantial movement of vessels in the zdirection. 


\section{Conclusion}

To summarize, this study revealed that the average individual vessel flow rates and total volumetric flow rates do not change for vessels of the same caliber post remodeling. Restoring blood flow through the saphenous artery (in silico) did not lead to any significant change in the total volumetric flow through the muscle. Connectivity changes and average diameters primarily change in the higher order vessels after remodeling. A few correlations could be made between architectural and flow properties, however, further modifications in the analysis methods can make future correlations more informative. The most important finding of this study is that the remodeling process is favorable to maintaining the average normal individual vessel flow rates, as well as volumetric flow rates. One useful change in the analysis method could be the use of ordering schemes that keep the vessel connectivity and diameter properties separate in grouping the vessels. Using a large number of samples as well as the parameter sensitivity analysis tool (PSAT) could make future studies more informative as well. The PSAT is helpful in teasing apart the individual effects of parameters such as vessel connectivity, vessel diameters, and vessel lengths. In future, another important component that allows the investigator to exclusively alter vessel quantities for all the orders can be added to improve the PSAT. Finally, more complex methods of modeling such as in COMSOL Multiphysics, can help in capturing the fine aspects of the vascular geometry and incorporate its effects in flow calculations without increasing the time and effort required for analysis. 


\section{Bibliography}

1. Al-Khaldi, A., H. Al-Sabti, et al. (2002). "Therapeutic angiogenesis using autologous bone marrow stromal cells: improved blood flow in a chronic limb ischemia model." Annals of Thoracic Surgery 75: 204-209.

2. Bailey, A. M., B. C. Thorne, et al. (2007). "Multi-cell agent-based simulation of the microvasculature to study the dynamics of circulating inflammatory cell trafficking." Annals of Biomedical Engineering 35(6): 916-936.

3. Can, A., H. Shen, et al. (1999). "Rapid automated tracing and feature extraction from retinal fundus images using direct exploratory algorithms." IEEE Transactions on Information Technology in Biomedicine 3(2): 125-138.

4. Cardinal, T. R. and J. B. Hoying (2007). "A modified fluorescent microspher-based approach for determining resting and hyperemic blood flows in individual murine skeletal muscles." Vascular Pharmacology 47(1): 48-56.

5. Carmeliet, P. and R. K. Jain (2000). "Angiogenesis in cancer and other diseases." Nature 407: 249-257.

6. Cebasek, V., I. Erzen, et al. (2010). "The estimation error of skeletal muscle capillary supply is significantly reduced by 3D method." Microvascular Research 79: 40-46.

7. Charalampidis, D., M. Pascotto, et al. (2006). "Anatomy and flow in normal and ischemic microvasculature based on a novel temporal fractal dimension analysis algorithm using contrast enhanced ultrasound." IEEE Transactions on Medical Imaging 25(8): 1079-1086.

8. Contreras, D. L., H. V. Robles, et al. (2006). "The role of nitric oxide in the postischemic revascularization process." Pharmacology and Therapeutics 112: 553-563.

9. Coyle, P. and D. D. Heistad (1987). "Blood flow through cerebral collateral vessels one month after middle cerebral artery occlusion." Stroke 18: 407-411.

10. Delp, M. D. and M. H. Laughlin (1998). "Regulation of skeletal muscle perfusion during exercise." Acta Physiologica Scandinavica 162: 411-419.

11. Fenton, B. M. and B. W. Zweifach (1981). "Microcirculatory model relating geometrical variation to changes in pressure and flow rate." Annals of Biomedical Engineering 9: 303-321.

12. Fournier, R. L. (1998). Basic Transport Phenomena in Biomedical Engineering, Taylor and Francis Group.

13. Frame, M. D. S. and I. H. Sarelius (1995). "Energy optimization and bifurcation angles in the microcirculation." Microvascular Research 50: 301-310. 
14. Gabhann, F. M., J. W. Ji, et al. (2007). "Multi-scale computational models of proangiogenic treatments in peripheral arterial disease." Annals of Biomedical Engineering 35(6): $982-94$.

15. Gruionu, G., J. B. Hoying, et al. (2005). "Structural remodeling of mouse gracilis artery after chronic alteration in blood supply." American Journal of Physiology Heart and Circulation Physiology 288: H2047-54.

16. Guyton and Hall (2006). Textbook of Medical Physiology, Elsevier Saunders.

17. Heil, M. and W. Schaper (2004). "Influence of mechanical, cellular, and molecular factors on collateral artery growth (arteriogenesis)." Circulation Research 95: 449-458.

18. Helisch, A. and W. Schaper (2003). "Arteriogenesis The development and growth of collateral arteries." Microcirculation 10: 83-97.

19. Hershey, J. C., E. P. Baskin, et al. (2001). "Revascularization in the rabbit hindlimb: dissociation between capillary sprouting and arteriogenesis." Cardiovascular Research 49: $618-25$.

20. Horsfield, K. (1976). "Some mathematical properties of branching trees with application to the respiratory system." Bulletin of Mathematical Biology 38: 305-315.

21. Hudlicka, O. (1982). "Growth of capillaries in skeletal and cardiac muscle." Circulation Research 50: 451-61.

22. Ince, C. (2005). "The microcirculation is the motor of sepsis." Critical Care 9: 13-19.

23. Ito, W. D., M. Arras, et al. (1997). "Monocyte chemotactic protein-1 increases collateral and peripheral conductance after femoral artery occlusion." Circulation Research 80: 829-837.

24. Jiang, Z. L., G. S. Kassab, et al. (1994). "Diameter-defined Strahler system and connectivity matrix of the pulmonary arterial tree." Journal of Applied Physiology 76: $882-892$

25. Kassab, G. S. (2000). "The coronary vasculature and its reconstruction." Annals of Biomedical Engineering 28(8): 903-915.

26. Kassab, G. S. (2005). "Scaling laws of vascular trees: of form and function." American Journal of Physiology Heart and Circulation Physiology 290: 894-903.

27. Kassab, G. S., J. Berkley, et al. (1997). "Analysis of pig's coronary arterial blood flow with detailed anatomical data." Annals of Biomedical Engineering 25: 204-217. 
28. Kaul, S. and A. R. Jayaweera (2006). "Determinants of microvascular flow." European Heart Journal 27(19): 2272-2274.

29. Kelsall, C. J., M. D. Brown, et al. (2004). "Arteriolar endothelial dysfunction is restored in ischemic muslces by chronic electrical stimulation." Journal of Vascular Research 41(3): 241-251.

30. Kitano, H. (2002). "Systems Biology: a brief overview." Science 295: 1662-64.

31. Krenz, G. S. and C. A. Dawson (2003). "Flow and pressure distributions in vascular networks consisting of distensible vessels." American Journal of Physiology Heart and Circulation Physiology 284: 2192-2203.

32. Lapi, D., P. L. Marchiafava, et al. (2008). "Geometric characteristics of arterial network of rat pial microcirculation." Journal of Vascular Research 45: 69-77.

33. Mayrovitz, H. N. and J. Roy (1983). "Microvascular blood flow: evidence indicating a cubic dependence on arteriolar diameter." American Journal of Physiology Heart and Circulation Physiology 245: H1031-H1038.

34. Mulvany, M. J. and C. Aalkjaer (1990). "Structure and function of small arteries." Physiological reviews 70: 921-61.

35. Peirce, S. M. and T. C. Skalak (2003). "Microvascular remodeling: a complex continuum spanning angiogenesis to arteriogenesis." Microcirculation 10: 99-111.

36. Pries, A. R., B. Reglin, et al. (2001). "Structural adaptation of microvascular networks: functional roles of adaptive responses." American Journal of Physiology Heart and Circulation Physiology 281: H1015-25.

37. Pries, A. R., B. Reglin, et al. (2001). "Structural adaptation of vascular networks: role of the pressure response." Hypertension 38: 1476-9.

38. Pries, A. R., B. Reglin, et al. (2003). "Structural response of microcirculatory networks to changes in demand: information transfer by shear stress." American Journal of Physiology Heart and Circulation Physiology 284: 2204-12.

39. Pries, A. R. and T. W. Secomb (2000). "Microcirculatory network structures and models." Annals of Biomedical Engineering 28: 916-921.

40. Pries, A. R. and T. W. Secomb (2002). "Structural Adaptation of Microvascular Networks and Development of Hypertension." Microcirculation 9(4): 305-314.

41. Pries, A. R. and T. W. Secomb (2005). "Control of blood vessel structure: insights from theoretical models." American Journal of Physiology Heart and Circulation Physiology 288: $1010-1015$. 
42. Pries, A. R., T. W. Secomb, et al. (1995). "Design principles of vascular beds." Circulation Research 77: 1017-1023.

43. Pries, A. R., T. W. Secomb, et al. (1995). "Structure and hemodynamic of microvascular networks: heterogeneity and correlations." American Journal of Physiology Heart and Circulation Physiology 269: H1713-22.

44. Pries, A. R., T. W. Secomb, et al. (1996). "Biophysical aspects of blood flow in the microvasculature." Cardiovascular Research 32: 654-667.

45. Pries, A. R., T. W. Secomb, et al. (1998). "Structural adaptation and stability of microvascular networks: theory and simulations." Microcirculation 275: 349-360.

46. Pries, A. R., T. W. Secomb, et al. (1990). "Blood flow in microvascular networks. Experiments and simulation." Circulation Research 67: 826-834.

47. Risau, W. (1997). "Mechanisms of Angiogenesis." Nature 386: 671-674.

48. Scholz, D., T. Ziegelhoeffer, et al. (2002). "Contribution of arteriogenesis and angiogenesis to postocclusive hindlimb perfusion in mice." Journal of Molecular and Cell Cardiology 34: 775-787.

49. Secomb, T. W., D. A. Beard, et al. (2008). "The Role of Theoretical Modeling in Microcirculation Research." Microcirculation 15: 693-698.

50. Skalak, T. C. and R. J. Price (1996). "The role of mechanical stresses in microvascular remodeling." Microcirculation 3(2): 143-165.

51. Skalak, T. C., R. J. Price, et al. (1998). "Where do new arterioles come from? Mechanical forces and microvessel adaptation." Microcirculation 5: 91-94.

52. Takeshita, S., T. Isshiki, et al. (1998). "Endothelium-dependent relaxation of collateral microvessels after intramuscular gene transfer of vascular endothelial growth factor in a rat model of hindlimb ischemia." Circulation Research 98: 1261-63.

53. Theodorakis, N. G., Y.-n. Wang, et al. (2003). "The role of nitric oxide synthase isoforms in extrahepatic portal hypertension: studies in gene-knockout mice." Gastroenterology 124(5): 1500-1508.

54. Unthank, J. L., C. Nixon, et al. (1995). "Early adaptations in collateral and microvascular resistances after ligation of the rat femoral artery." Journal of Applied Physiology 79: 7382.

55. Unthank, J. L., K. M. Sheridan, et al. (2004). "Collateral growth in the peripheral circulation: a review." Vascular and Endovascular Surgery 38: 291-313. 
56. Wahlberg, E. (2003). "Angiogenesis and arteriogenesis in limb ischemia." Journal of Vascular Surgery 38: 198-203.

57. Wiedeman, M. P. (1968). "Blood flow through terminal arterial vessels after denervataion of the bat wing." Circulation Research 22: 83-90.

58. Yang, H. T., M. R. Deschenes, et al. (1996). "Basic fibroblast growth factor increases collateral blood flow in rats with femoral arterial ligation." Circulation Research 79: 6269.

59. Zamir, M. (1976). "The role of shear forces in arterial branching." Journal of General Physiology 67: 213-22.

60. Zweifach, B. W., S. Kovalcheck, et al. (1981). "Micropressure-flow relationships in a skeletal muscle of spontaneously hypertensive rats." Hypertension 3: 601-614.

61. Zweifach, B. W. and H. H. Lipowsky (1977). "Quantitative studies of microcirculatory structure and function. III. Microvascular hemodynamics of cat mesentery and rabbit omentum." Circulation Research 41: 380-390. 
Appendices 


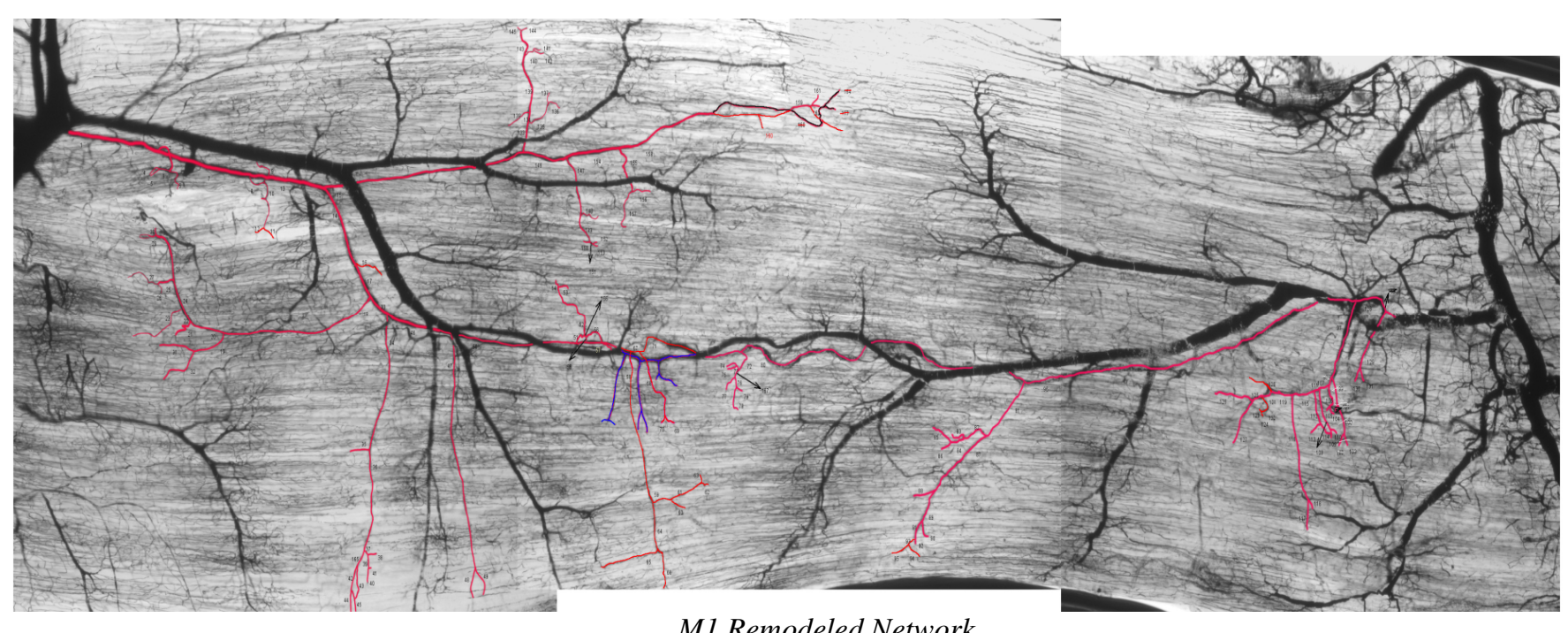

M1 Remodeled Network 


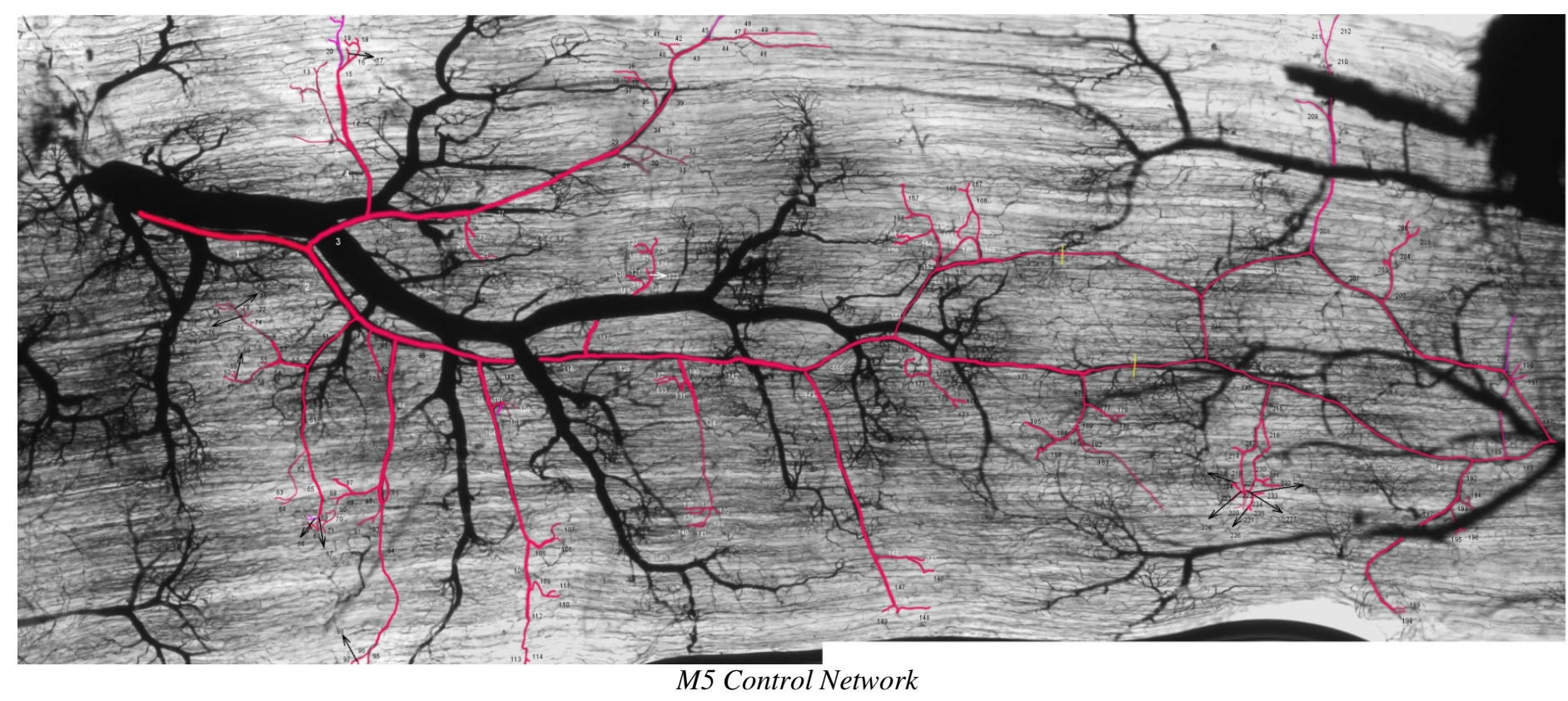




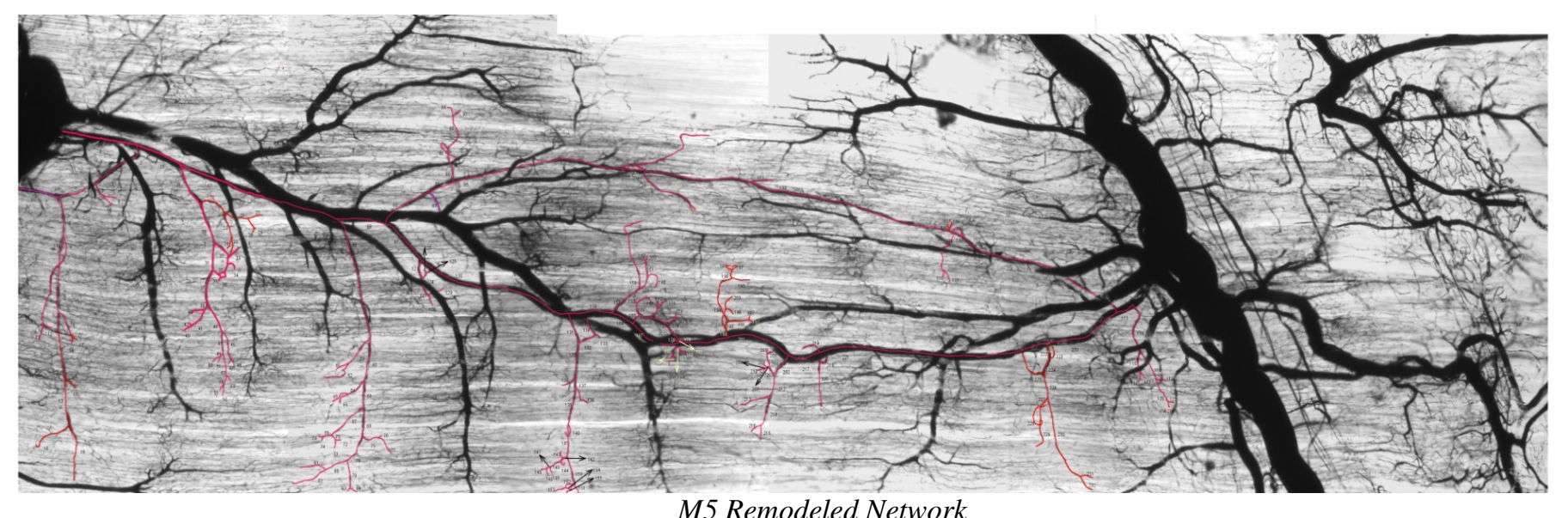




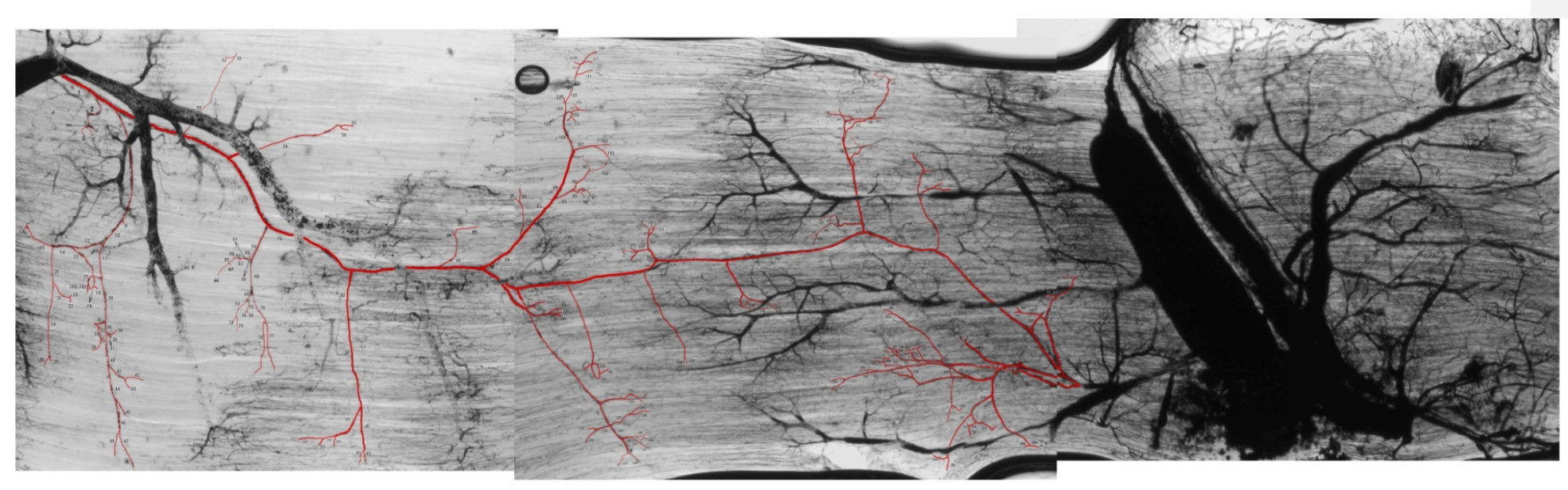

M6 Control Network 


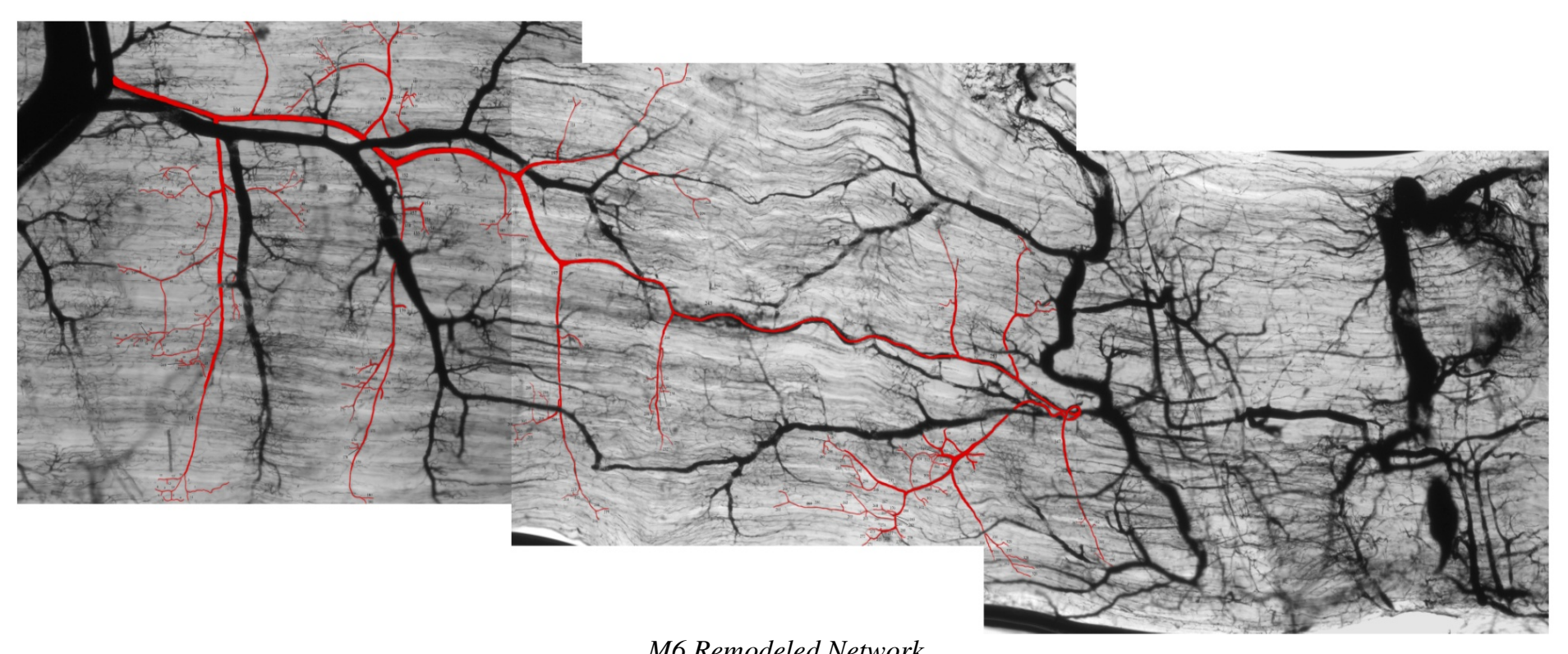




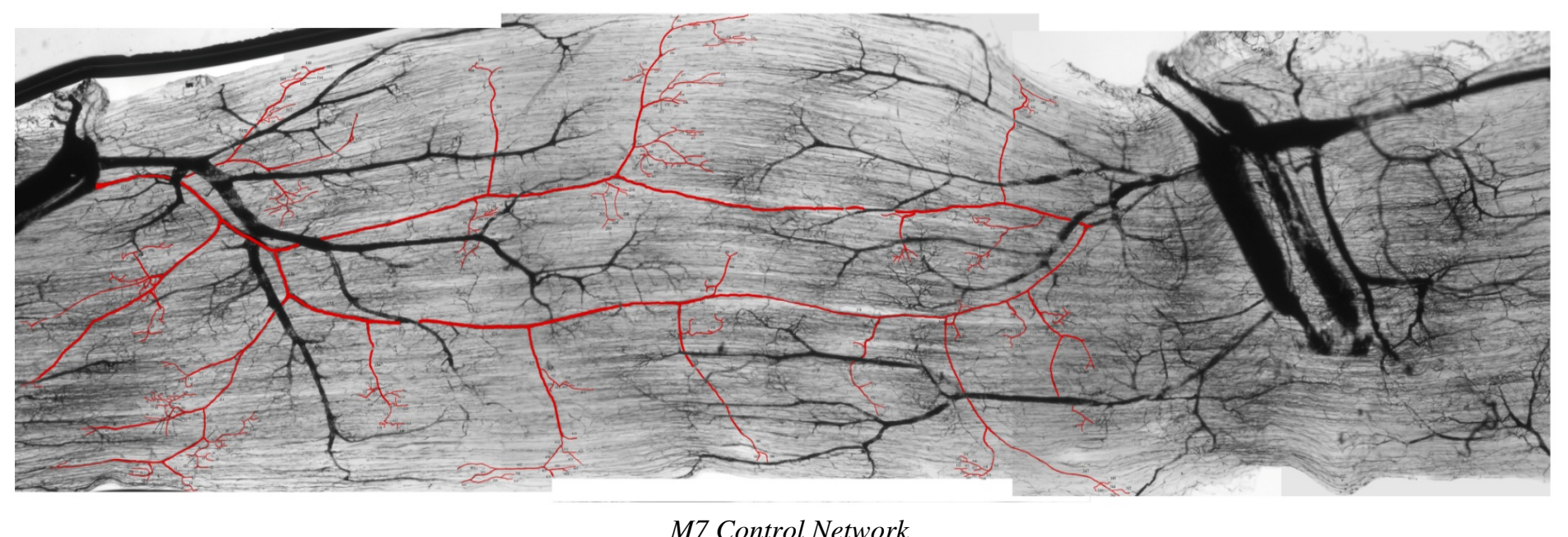

M7 Control Network 


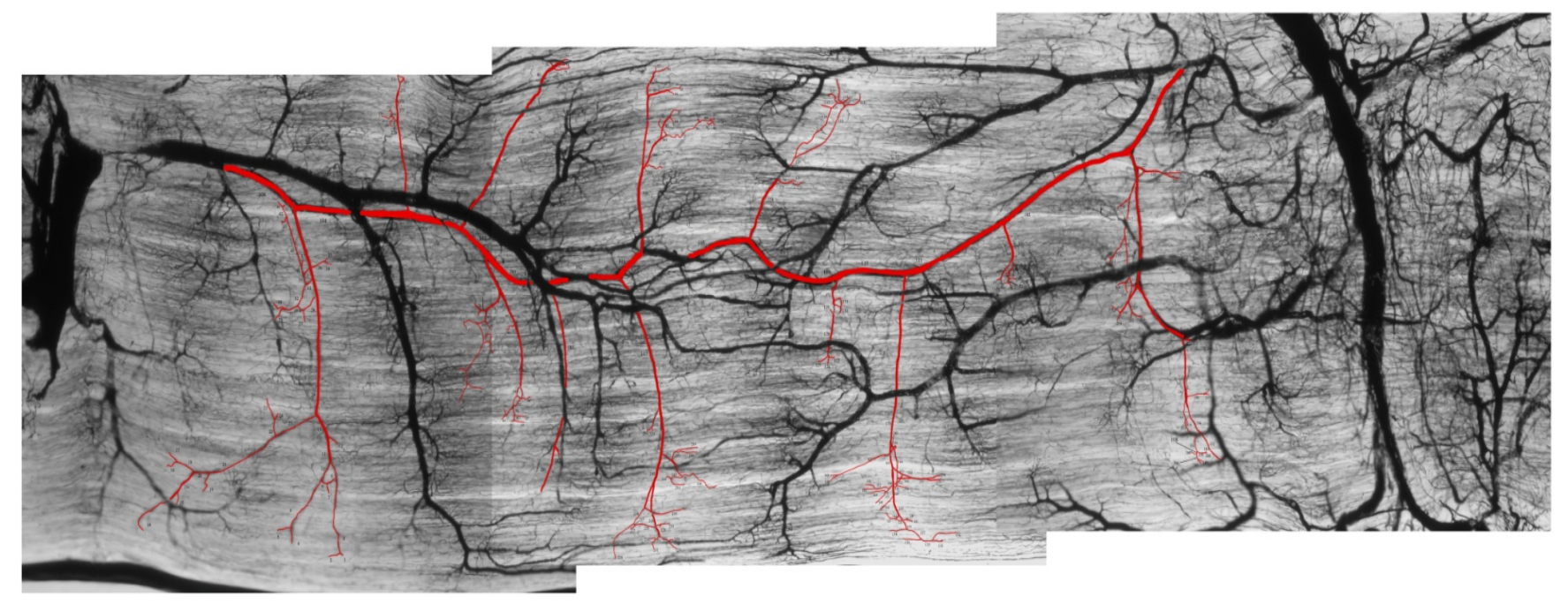

M7 Remodeled Network 


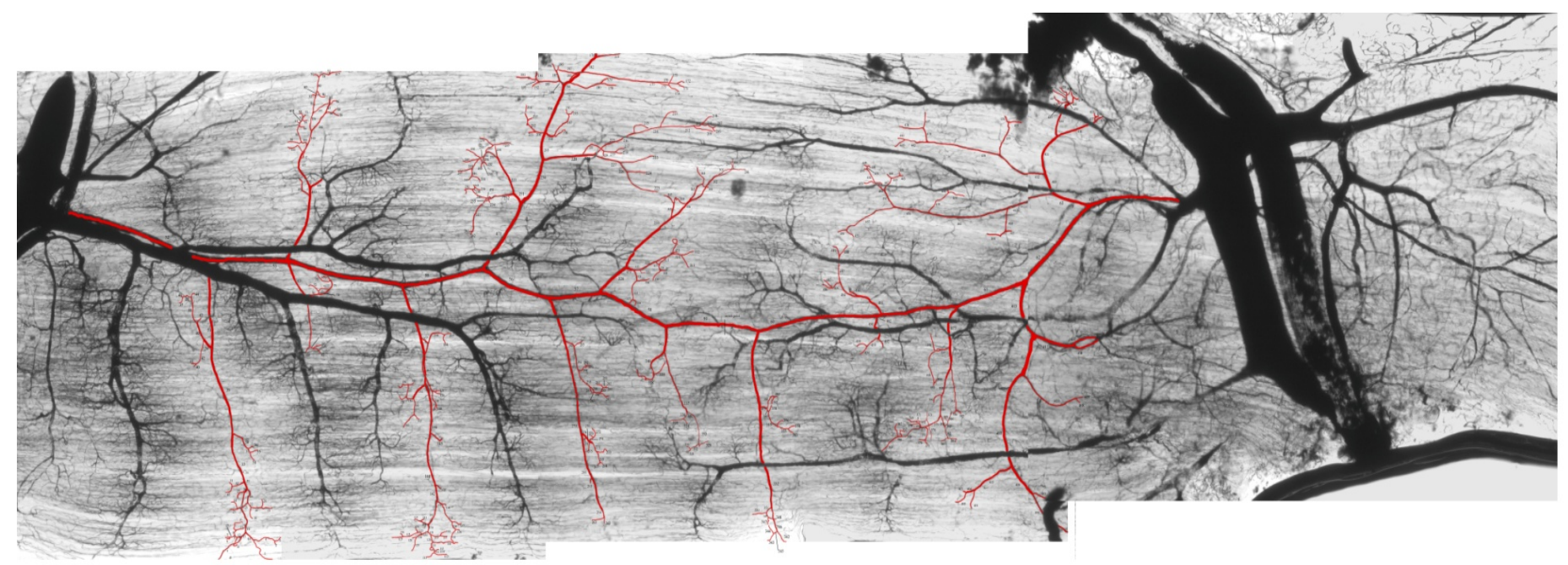

M8 Control Network 


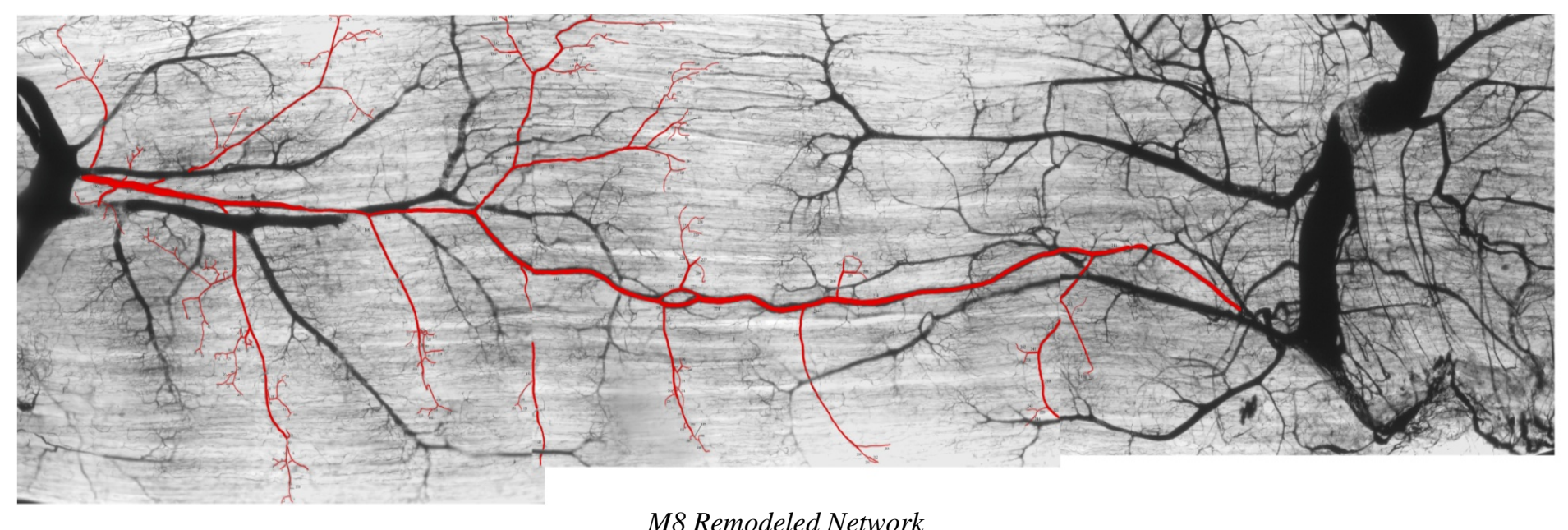


\%The connectivity code is given a disassembled network of vessel segments whose diameters and lengths have been left unchanged.

- The code then reassembles all the segments by joining the segments end-to-end according to user-defined rules.

oThe user provides the desired branching characteristics of the network by inputting the percentage values of daughter segments of a particular order that arise from parent segments of a particular order.

$\because D E F I N E$ VARIABLES

AVAILOFF1S=1; AVAILOFF2S=1; AVAILOFF3S=1; AVAILOFF1S=1; AVAILOFF4S=1; AVAILOFF5S=1; $\frac{\circ}{\circ}$ AVAILOFFS Variables indicate the position (matrix row) from where new offspring segments are available

AVAILOFF2E=79; AVAILOFF3E=36; AVAILOFF4E=5; AVAILOFF5E=14; $\frac{\circ}{\circ}$ AVAILOFFE variables indicate the position (matrix row) at which number of available offspring segments end

AVAILPAR2 $=0 ;$ AVAILPAR3=0;AVAILPAR4=0;AVAILPAR5=0; AVAILPAR variables indicate the position (matrix row) from where available parent segments begin

LAST_ROW_OF_MATRIX_TWO $=79$; LAST_ROW_OF_MATRIX_THREE=36; $\%$ the number of rows in each matrix (total number of available segments)

LAST_ROW_OF_MATRIX_FOUR=5; LAST_ROW_OF_MATRIX_FIVE $=14$;

\% number of specific branch types (each one is illustrated later in the code)

$\mathrm{V}=60$;

$\mathrm{w} 1=84 ; \mathrm{w} 2=20 ; \mathrm{w} 3=14 ;$

$\mathrm{x} 1=16 ; \mathrm{x} 2=4 ; \mathrm{x} 3=14 ; \mathrm{x} 4=0 ; \mathrm{x} 5=8 ; \mathrm{x} 6=8$;

$\mathrm{y} 1=0 ; \mathrm{y}^{2}=0 ; \mathrm{y}^{3}=0 ; \mathrm{y}^{4}=2 ; \mathrm{y} 5=1 ; \mathrm{y} 6=4 ; \mathrm{y} 7=0 ; \mathrm{y} 8=1 ; \mathrm{y} 9=0 ; \mathrm{y} 10=0 ;$

$\mathrm{z} 1=0 ; \mathrm{z} 2=0 ; \mathrm{z3}=0 ; \mathrm{z} 4=0 ; \mathrm{z} 5=0 ; \mathrm{z} 6=0 ; \mathrm{z} 7=1 ; \mathrm{z} 8=5 ; \mathrm{z9}=3 ; \mathrm{z} 10=4 ;$

orange of available offspring segment

RANGE2 = AVAILOFF2E - AVAILOFF $2 S+1$

RANGE3 = AVAILOFF3E - AVAILOFF3S +1;

RANGE4 = AVAILOFF4E - AVAILOFF4S +1;

RANGE5 = AVAILOFF5E - AVAILOFF5S +1;

OREAD MATRICES FROM SPREADSHEET

Vessel segment labels are read from each excel spreadsheet into the

omatrices. Each matrix has 4 columns. The first column contains the segment

olabels, the second column contains the parent labels, the third column 
\%contains the number of offspring of the corresponding segments, and the ofourth column contains the segment order numbers.

One $=$ xlsread('Thesis spreadsheet with m6 remodeled data (control order bounds).xlsm', 'Matlab input 1'); Two $=$ xlsread('Thesis spreadsheet with m6 remodeled data (control order bounds).xlsm', 'Matlab input 2'); Three $=$ xlsread('Thesis spreadsheet with m6 remodeled data (control order bounds).xlsm','Matlab input 3')

Four = xlsread ('Thesis spreadsheet with m6 remodeled data (control order bounds). xlsm', 'Matlab input 4');

Five $=$ xlsread('Thesis spreadsheet with m6 remodeled data (control order bounds).xlsm', 'Matlab input 5');

응

OASSIGN PARENT ORDER 1 BRANCHES

if $\mathrm{V} \sim=0$ \% checks to see whether there are any 1-1-1 branch types

$\mathrm{c} 1=1$;

for $a=1: 2: \mathrm{v}$

One $(a, 4)=$ One $(v+c 1,1)$;

One $(a+1,4)=$ One $(v+c 1,1) ; \%$

One $(\mathrm{v}+\mathrm{C} 1,3)=2$;

$\mathrm{c} 1=\mathrm{C} 1+1 ;$;

end

AVALLOFF $1 S=\quad v+1$

clear a;

else

end

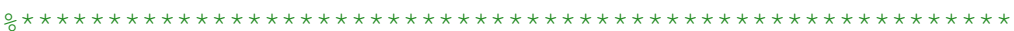

$\because$ ASSIGN PARENT ORDER 2 BRANCHES

if $\mathrm{w} 1 \sim=0$ \% checks to see whether there are any 2-1-1 branch types

$\mathrm{c} 2=1 ;$ pos1 = AVAILOFF1S; pos2 = AVAILOFF1S + w1-2;

for $a=\operatorname{pos} 1: 2:$ pos 2

One $(a, 4)=$ Two (AVAILPAR2 $+c 2,1) ;$

One $(a+1,4)=$ Two $($ AVAILPAR2 $+c 2,1)$; Two $($ AVAILPAR2 $+c 2,3)=2 ; \%$

$\mathrm{c} 2=\mathrm{c} 2+1$;

end $\frac{\circ}{\circ}$

AVAILOFF1S = AVAILOFF1S + w1;

AVAILPAR2 = AVAILPAR2 + $22-1$

AVAILOFF2E = AVAILPAR2;

RANGE2=AVAILOFF 2E-AVAILOFF $2 \mathrm{~S}+1$

clear a; 
if $\mathrm{w} 2 \sim=0 \%$ checks to see whether there are any 2-1-2 branch types

check = AVAILPAR2 + 1

if AVAILOFF2S $==1$ \&\& check $==1$

AVAILPAR2 = w2; AVAILOFF2E = w2; RANGE2 = AVAILOFF2E - AVAILOFF2S;

end

$\mathrm{c} 3=1 ;$ pos1=AVAILOFF1S; pos2=AVAILOFF1S+w2-1;

for $a=\operatorname{pos} 1:$ pos 2 .

One $(a, 4)=$ Two $(\operatorname{AVAILPAR} 2+c 3,1) ;$ Two $(\operatorname{AVAILPAR} 2+c 3,3)=1$;

$c 3=c 3+1$;

end

AVAILOFF1S $=$ pOs $2+1$

AVAILNEW2 = AVAILPAR2 +c3

clear $a ; c 3=1$

if w2<RANGE2 \%checks to see whether range of available offspring is greater than the required number of offspring

pos1=AVAILOFF $2 \mathrm{~S} ;$ pos2=AVAILOFF $2 \mathrm{~S}$ +w2-1;

for $a=$ pos $1:$ pos 2

$\operatorname{Two}(a, 4)=\operatorname{Two}(\operatorname{AVAILPAR} 2+\mathrm{c} 3,1) ; \operatorname{Two}(\operatorname{AVAILPAR} 2+\mathrm{c} 3,3)=2$;

$c 3=c 3+1$

end

AVAILOFF $2 \mathrm{~S}=$ pOS $2+1$;

AVAILPAR2=AVAILPAR2+C3 -1 ; AVAILOFF2E = AVAILPAR2

AVAILNEW2=AVAILPAR2+1

RANGE2=AVAILOFF 2E-AVAILOFF $2 \mathrm{~S}+1$

c3 $=1$; clear a

else

if $\mathrm{w} 2==$ RANGE2\%checks to see whether range of available offspring is equal to the required number of offspring

pos1=AVAILOFF $2 \mathrm{~S} ;$ pos2 = AVAILOFF2S + RANGE2-1;

for $a=$ posi:pos2

$\operatorname{Two}(a, 4)=\operatorname{Two}(\operatorname{AVAILPAR} 2+c 3,1) ; \operatorname{Two}(\operatorname{AVAILPAR} 2+c 3,3)=2$;

$\mathrm{c} 3=\mathrm{c} 3+1$

end

off $=$ pos $2+1$ 
while Two (off, 4$) \sim=0$

off $=$ off +1 ;

AVAILOFF2S = off;AVAILOFF2E = AVAILPAR2 + $33-1 ;$

AVAILPAR2 $=$ AVAILPAR $2+C 3-1$

RANGE2=AVAILOFF 2E-AVAILOFF $2 \mathrm{~S}+1$;

c3=1; clear a;

else

if w2>RANGE $2 \%$ checks to see whether range of available offspring is less than the required number of offspring

pOS1=AVAILOFF2S; pos2 = AVAILOFF2S + RANGE2 -1;

for $a=$ pos $1:$ pos 2

$\operatorname{Two}(a, 4)=\operatorname{Two}($ AVAILPAR2 $+\mathrm{c} 3,1)$; Two $($ AVAILPAR2 $+\mathrm{c} 3,3)=2$;

$\mathrm{c} 3=\mathrm{c} 3+1$

end

AVAILOFF $2 \mathrm{~S}=$ AVAILPAR2 $+\mathrm{w} 2+1 ;$

AVAILOFF2E = LAST_ROW_OF_MATRIX_TWO;

clear a;

num = $\mathrm{w} 2$ - RANGE2;

$\mathrm{C} 3=1 ;$ pos $1=$ AVAILOFF $2 \mathrm{~S} ;$ pos2 = AVAILOFF $2 \mathrm{~S}+$ num $-1 ;$

for $a=$ pos $1:$ pos2

$\operatorname{Two}(a, 4)=$ Two $(\operatorname{AVAILPAR} 2+c 3,1) ;$ Two $(\operatorname{AVAILPAR2}+\mathrm{c} 3,3)=2$;

$\mathrm{c} 3=\mathrm{c} 3+1$;

end

AVAILOFF2S = AVAILPAR2 +1;

AVAILPAR2 = AVAILPAR2 + $\mathrm{C} 3-1$

AVAILNEW2 = pOS2 +1;

AVAILOFF2E = AVAILPAR2;

clear a;

RANGE2= AVAILOFF2E-AVAILOFF2S +1;

end

end

end

end

if w3 =0\% checks to see whether there are any 2-2-2 branch types

check = AVAILPAR2 + 1

if AVAILOFF2S $==1$ \&\& check $==1$ 
AVAILPAR2 = w3; AVAILOFF2E = w3; RANGE2 = AVAILOFF2E-AVAILOFF2S +1;

end

$\mathrm{c} 5=1$

if $w 3<$ RANGE $2 \%$ checks to see whether range of available offspring is greater than the required number of offspring

pos1 = AVALLOFF2S; pos2 = AVAILOFF2S +w3 -1; $\mathrm{a}=$ pos1;

while a $<$ pos 2

$\operatorname{Two}(a, 4)=$ Two $(\operatorname{AVAILPAR} 2+\mathrm{c} 5,1) ;$

Two $(a+1,4)=$ Two $($ AVAILPAR2 $+c 5,1) ;$ Two $($ AVAILPAR $2+c 5,3)=2 ;$

$c 5=c 5+1 ; a=a+2$

end

AVAILOFF $2 \mathrm{~S}=$ pOS $2+1$;

AVAILPAR2 = AVAILPAR2 + C5 -1;AVAILOFF2E = AVAILPAR2;

RANGE2 = AVAILOFF2E - AVAILOFF $2 \mathrm{~S}+1 ;$

AVAILNEW2 = AVAILPAR2 +1;

clear a;

else

if $\mathrm{w} 3==$ RANGE2\%checks to see whether range of available offspring is equal to the required number of offspring

pos1 = AVAILOFF2S; pos2 = AVAILOFF2S + RANGE2 -1; $\mathrm{a}=$ pos $1 ;$

while a $<$ pos2

Two $(a, 4)=$ Two $($ AVAILPAR2 $+c 5,1)$.

Two $(a+1,4)=$ Two $($ AVAILPAR2 $+c 5,1)$; Two $($ AVAILPAR2 $+c 5,3)=2$;

$c 5=c 5+1 ; a=a+2$;

end

off $=\operatorname{pos} 2+1$;

while Two $($ off, 4$) \sim=0$

off $=$ off +1

end

AVAILOFF2S = Off; AVAILOFF2E = AVAILPAR2 + C5-1;

AVAILPAR2 = AVAILPAR2 + c5 - 1; c5 =1; clear $a ;$

RANGE2 = AVAILOFF2E - AVAILOFF $2 S+1$

else

if w3>RANGE2\%checks to see whether range of available offspring is less than the required number of offspring

if $\quad r e m(R A N G E 2,2)==0$

pos $1=$ AVAILOFF $2 \mathrm{~S} ;$ pos $2=$ AVAILOFF $2 \mathrm{~S}+\operatorname{RANGE} 2-1 ; \mathrm{a}=$ pos $1 ;$ 
while a $<$ pos 2

$\operatorname{Two}(a, 4)=$ Two $(\operatorname{AVAILPAR} 2+c 5,1) ;$

Two $(a+1,4)=$ Two (AVAILPAR2 $+c 5,1) ;$ Two (AVAILPAR2 $+c 5,3)=2 ;$

$c 5=c 5+1 ; a=a+2$;

end

AVAILOFF2S = AVAILPAR2 + (w3/2) +1;

AVAILOFF2E = LAST_ROW_OF_MATRIX_TWO;

num $=$ w3 - RANGE2; clear a;

pos1 = AVAILOFF2S; pos2 = AVAILOFF $2 \mathrm{~S}+$ num $-1 ; \mathrm{a}=\operatorname{pos} 1 ;$

while a $<$ pos2

$\operatorname{Two}(a, 4)=\operatorname{Two}(\operatorname{AVAILPAR} 2+c 5,1) ;$

Two $(a+1,4)=$ Two $($ AVAILPAR2 $+c 5,1)$; Two (AVAILPAR2 $+c 5,3)=2$;

c5 $=c 5+1 ; a=a+2$;

end

clear a;

else

pos1 = AVAILOFF2S; pos2 = AVAILOFF $2 S+$ RANGE2 $-1 ; \mathrm{C}=0$

for $a=$ pos $1:$ pos 2

$\mathrm{C}=\mathrm{C}+1$;

$\operatorname{Two}(a, 4)=$ Two $($ AVAILPAR2 $+c 5,1) ;$ Two $($ AVAILPAR2 $+c 5,3)=c$;

if Two (AVAILPAR2 $+c 5,3)==2$ $\mathrm{C} 5=\mathrm{C} 5+1 ; \mathrm{C}=0$;

end

end

AVAILOFF2S = AVAILPAR2 + (w3/2) +1

AVAILOFF2E = LAST_ROW_OF_MATRIX_TWO;

num $=$ w3 - RANGE2; clear a;

pOS1 $1=$ AVAILOFF2S; pOS2 = AVAILOFF $2 \mathrm{~S}+$ num -1

for $a=$ posi:pos2

$\mathrm{C}=\mathrm{C}+1$;

Two $(a, 4)=$ Two $($ AVAILPAR2 $+c 5,1)$; Two $($ AVAILPAR2 $+c 5,3)=c$;

if Two (AVAILPAR2 $+c 5,3)==2$

end

$\mathrm{C}=\mathrm{C} 5+1 ; \mathrm{C}=0$;

end
end
end
end
end
end 
if $\mathrm{x} 1 \sim=0 \%$ checks to see whether there are any $3-1-1$ branch types $\mathrm{c} 6=1 ;$ pos 1 = AVAILOFF1S; pos 2 = AVAILOFF1S +x1-2;

for $a=\operatorname{pos} 1: 2:$ pos $2 \%$

One $(a, 4)=$ Three $($ AVAILPAR $3+c 6,1) ; \circ$

One $(a+1,4)=$ Three $($ AVAILPAR3 $+c 6,1) ;$ Three $($ AVAILPAR3 $+c 6,3)=2$; $c 6=c 6+1 ;$

end

AVAILOFF1S $=$ AVAILOFF $1 S+\mathrm{x} 1$;

AVAILPAR3 = AVAILPAR3 + $66-1 ;$

AVAILOFF3E= AVAILPAR3;

RANGE3 = AVAILOFF3E - AVAILOFF3S +1;

clear a;

end

if $\times 2 \sim=0 \div$ checks to see whether there are any $3-1-2$ branch types $\mathrm{C} 7=1 ;$ pos $1=$ AVAILOFF $1 \mathrm{~S} ;$ pos $2=$ AVAILOFF1S + $22-1 ; \%$

for $a=\operatorname{pos} 1:$ pos 2 \%

One $(a, 4)=$ Three $($ AVAlLPAR3 $+c 7,1)$; Three $($ AVALLPAR3 $+c 7,3)=1$; $\mathrm{c} 7=\mathrm{c} 7+1$;

end

AVAILOFF1S $=$ pos $2+1$

clear a; $\mathrm{c} 7=1 ;$ num $=\mathrm{x} 2+1$;

for $a=1$ : LAST_ROW_OF_MATRIX_TWO

if $\operatorname{Two}(a, 4)==0 \& \& \quad c 7<$ num

$\operatorname{Two}(a, 4)=$ Three $($ AVAILPAR3 $+c 7,1)$; Three $(\operatorname{AVAILPAR3}+\mathrm{c} 7,3)=2$;

$c 7=c 7+1$

end

end

AVAILPAR3 $=$ AVAILPAR3 + C7 $-1 ;$

AVAILOFF3E = AVAILPAR3;

RANGE3 = AVAILOFF3E - AVAILOFF3S + ;

clear a;

end 
if $x 3 \sim=0 \div$ checks to see whether there are any 3-2-2 branch types

$\mathrm{c} 8=1 ;$ num $=(\times 3 / 2)+1 ; \mathrm{C}=0$;

for $a=1$ : LAST ROW OF MATRTX TWO

if $\operatorname{Two}(a, 4)==0$ \&\& $\mathrm{c} 8<$ num

$\mathrm{C}=\mathrm{C}+1 ; \%$

Two $(a, 4)=$ Three $($ AVAILPAR3 $+c 8,1)$; Three (AVAILPAR3 + c8, 3 ) = c ;

if Three (AVAILPAR3 $+c 8,3)==2$

$\mathrm{c} 8=\mathrm{c} 8+1 ; \mathrm{c}=0$;

end

end

end

AVAILPAR3 = AVAILPAR3 $+\mathrm{C} 8-1$

AVAILOFF3E = AVAILPAR3;

RANGE3 = AVAILOFF3E - AVALLOFF3S +

clear a;

end

if $\mathrm{x} 4 \sim=0$ \% checks to see whether there are any 3-1-3 branch types check = AVAILPAR3 $+1 ; \%$

if AVAILOFF3S == 1 \&\& check $==1 \%$

AVAILPAR3 $=x 4 ;$ AVAILOFF $3 E=x 4 ;$ RANGE3 = AVAILOFF3E-AVAILOFF3S + 1 ;

end \%

C9 $=1 ; \operatorname{pos} 1=$ AVAILOFF1S;

pos2 = AVAILOFF1S $+\mathrm{x} 4-1$;

for $a=$ pos $1:$ pos 2

One $(a, 4)=$ Three $($ AVAILPAR3 $+c 9,1)$; Three $($ AVAILPAR3 $+c 9,3)=1$

$c 9=c 9+1$

end

clear a; AVAILOFF1S = pos $2+1$

if $\mathrm{x} 4<$ RANGE3\%checks to see whether range of available offspring is greater than the required number of offspring

$\mathrm{C} 9=1$;

pos1 = AVAILOFF3S; pos2 = AVAILOFF3S $+\mathrm{x} 4-1 ;$

for $a=$ posi:pos 2

Three $(a, 4)=$ Three (AVAILPAR3 +c9, 1$)$; Three (AVAILPAR3 + c9, 3) $=2$;

c9 9 c $9+1$

end

AVALLOFF $3 S=\operatorname{pos} 2+1$ 
AVAILPAR3 = AVAILPAR3 + C9 - 1; AVAILOFF3E = AVAILPAR3;

RANGE3 = AVAILOFF3E - AVALLOFF3S +1;

clear a;

else

if $\mathrm{x} 4==$ RANGE $3 \%$ checks to see whether range of available offspring is equal to the required number of offspring

$\mathrm{c} 9=1$;

pos1 = AVALLOFF3S; pos2 = AVAILOFF3S + RANGE3 -1;

for $a=$ posi:pos2

Three $(a, 4)=$ Three (AVALLPAR3 $+c 9,1) ;$ Three (AVAILPAR3 $+c 9,3)=2$

$c 9=c 9+1$;

end

off $=$ pos $2+1$

while Three (off, 4) $\sim=0$

off $=$ off +1 ;

end

AVAILOFF3S $=$ off;

AVAILOFF3E = AVAILPAR3 + $99-1 ;$

AVAILNEW3 = AVAILPAR3 + C9;

AVAILPAR3 = AVAILNEW3 $-1 ; c 9=1 ;$ clear $a$;

RANGE3 = AVAILOFF3E - AVAILOFF3S +1;

else

if $\mathrm{x} 4>\mathrm{RANGE} 3 \% \mathrm{checks}$ to see whether range of available offspring is less than the required number of offspring

$\mathrm{c} 9=1$;

pos1 = AVAILOFF3S; pos2 = AVAILOFF3S + RANGE3 -

for $a=$ pos $1:$ pos 2

Three $(a, 4)=$ Three (AVAILPAR3 $+c 9,1)$; Three $($ AVAILPAR3 $+c 9,3)=2$;

$c 9=c 9+1$

end

AVAILOFF3S $=$ AVAILPAR3 $+x 4+1 ;$

AVAILOFF3E = LAST_ROW_OF_MATRIX_THREE;

num $=\mathrm{x} 4-$ RANGE3; clear a

pos1 = AVALLOFF3S; pos2 = AVALLOFF $3 S+$ num -1

for $a=p o s 1: p o s 2$

Three $(a, 4)=$ Three $($ AVAILPAR3 $+c 9,1)$; Three $($ AVAILPAR3 $+c 9,3)=2$;

$\mathrm{C} 9=\mathrm{C} 9+1$;

end 
AVAILOFF $3 S=$ AVAILPAR3 +1

AVAILPAR3 = AVAILPAR3 + C9 -1

AVAILNEW3 = pos2 +1; clear a;

AVAILOFF3E = AVAILPAR3;

RANGE3 = AVALLOFF3E - AVALLOFF3S +

end

end

end

end

if $\mathrm{x} 5 \sim=0$ \% checks to see whether there are any 3-2-3 branch types

check = AVAILPAR3 $+1 ;$

if AVAILOFF3S $==1$ \&\& check $==1$ \%

AVAILPAR $3=\times 5$; AVATLOFF $3 E=\times 5 ;$ RANGE $3=$ AVATLOFF $3 E-A V A I L O F F 3 S+1$,

end $\%$

$\mathrm{c} 10=1$; num $=\times 5+1 ; \%$

for $a=1$ : LAST_ROW_OF_MATRIX_TWO

if $\operatorname{TwO}(a, 4)==0$ \&\& $\quad 10<$ num

Two $(a, 4)=$ Three $($ AVAILPAR3 $+c 10,1)$; Three $($ AVAILPAR3 $+c 10,3)=1$;

$\mathrm{c} 10=\mathrm{c} 10+1$

end

end

clear a;

if $x 5<$ RANGE $3 \%$ checks to see whether range of available offspring is greater than the required number of offspring

$$
\mathrm{C} 10=1 ;
$$

pos1 = AVAILOFF3S; pos2 = AVAILOFF3S +x5 -1;

for $a=$ posi:pos2

Three $(a, 4)=$ Three $($ AVAILPAR3 $+\mathrm{c} 10,1)$; Three $($ AVAILPAR3 $+\mathrm{c} 10,3)=2$

$\mathrm{c} 10=\mathrm{c} 10+1$

end

AVAILOFF3S $=$ pOS $2+1 ;$

AVAILPAR3 = AVAILPAR3 + c10 - 1; C10 =1;AVAILOFF3E =AVAILPAR3;

RANGE3 = AVAILOFF3E-AVAILOFF3S +1

else

if $\mathrm{x} 5$ ==RANGE3\%checks to see whether range of available offspring is equal to the required number of offspring 
$\mathrm{C} 10=1 ;$

pos1 = AVAILOFF3S; pos2 = AVAILOFF3s +x5 -1;

for $a=$ posi:pos 2

Three $(a, 4)=$ Three (AVAILPAR3 $+\mathrm{c} 10,1)$; Three (AVAILPAR3 $+\mathrm{c} 10,3)=2$;

$\mathrm{c} 10=\mathrm{c} 10+1$

end

off $=$ pos $2+1$

while Three (off, 4) $\sim=0$

off $=$ off +1 ;

end

AVAILOFF3S $=$ off

AVAILOFF $3 E=$ AVAILPAR3 + C10 $-1 ;$

AVAILPAR3 $=$ AVAILPAR3 $+\mathrm{C} 10-1 ;$

RANGE3 = AVAILOFF3E - AVAILOFF3S + $i$

c10 = 1; clear $a$;

else

if $x 5>$ RANGE $3 \%$ checks to see whether range of available offspring is less than the required number of offspring

$$
\mathrm{C} 10=1 ;
$$

pos1 = AVALLOFF3S; pos2 = AVALLOFF3S + RANGE3 -1;

for $a=$ posi:pos 2

Three $(a, 4)=$ Three $($ AVAILPAR3 $+c 10,1)$; Three $($ AVAILPAR3 $+c 10,3)=2 ;$

$\mathrm{c} 10=\mathrm{c} 10+1$

end

AVAILOFF3S $=$ AVAILPAR3 $+x 5+1$

AVAILOFF3E = LAST_ROW_OF_MATRIX_THREE;

num $=x 5-$ RANGE3; clear a;

pOS1 = AVAILOFF3S; pOS2 = AVAILOFF3S + num -1;

for $a=$ posi:pos2

Three $(a, 4)=$ Three $($ AVAILPAR3 $+\mathrm{c} 10,1) ;$ Three $($ AVAILPAR3 $+\mathrm{c} 10,3)=2$;

$\mathrm{c} 10=\mathrm{c} 10+1$

end

AVAILOFF3S = AVAILPAR3 +1;

AVAILPAR3 = AVAILPAR3 + $\mathrm{c} 10-1 ;$

AVAILOFF3E = AVAILPAR3;

RANGE3 = AVAILOFF3E - AVAILOFF3S +

end

end

end

end 
if $x 6 \sim=0$ 을 check = AVAILPAR3 +1

if $\mathrm{AVAILOFF} 3 \mathrm{~S}==1$ \&\& Check $==1 \%$

AVAILPAR3 = x6; AVAILOFF3E = x6; RANGE3 = AVAILOFF3E-AVAILOFF3S $+1 ; \%$

end \%

$\mathrm{c} 11=1 ; \%$

3
1
$3^{1}$

if $x 6<$ RANGE $\%$ checks to see whether range of available offspring is greater than the required number of offspring

pos1 = AVAILOFF3S; pos2 = AVAILOFF3S +x6 -1; a = pOS $1 ;$

while a pos2

Three $(a, 4)=$ Three $($ AVAILPAR3 $+\mathrm{c} 11,1)$;

Three $(a+1,4)=$ Three $($ AVAILPAR3 $+c 11,1) ;$ Three $($ AVAILPAR3 $+c 11,3)=2$

$\mathrm{c} 11=\mathrm{c} 11+1 ; \mathrm{a}=\mathrm{a}+2$

end

AVAILOFF $3 S=$ pOS $2+1$

AVAILPAR3 = AVAILPAR3 + 11 -1;AVAILOFF3E = AVAILPAR3

RANGE3 = AVAILOFF3E - AVAILOFF $3 S+1$

AVAILNEW3 = AVALLPAR3 +1;

clear a;

else

if $\mathrm{x} 6==$ RANGE3\%checks to see whether range of available offspring is equal to the required number of offspring

pos1 = AVAILOFF3S; pos2 = AVAILOFF3S + RANGE3 $-1 ; a=$ pos $1 ;$

while a pos2

Three $(a, 4)=$ Three (AVAILPAR3 + c11, 1)

Three $(a+1,4)=$ Three $($ AVAILPAR3 $+c 11,1)$; Three $($ AVAILPAR3 $+c 11,3)=2$

$\mathrm{c} 11=\mathrm{c} 11+1 ; \mathrm{a}=\mathrm{a}+2$;

off $=$ pos $2+1$

while Three (off, 4) $\sim=0$

off $=$ off +1

end

AVAILOFF3S = off; AVAILOFF3E = AVAILPAR3 + C11-1;

AVAILPAR3 = AVAILPAR3 + c11 - 1; c11 =1; clear $a ;$

RANGE3 = AVAILOFF3E - AVAILOFF3S +

else 
if $x 6>$ RANGE3\%checks to see whether range of available offspring is less than the required number of offspring

$$
\text { if } r e m(R A N G E 3,2)==0
$$

pos1 = AVAILOFF 3 S $;$ pos $2=$ AVAILOFF $3 S+\operatorname{RANGE} 3-1 ; \mathrm{a}=$ pos $1 ;$

while a pos2

Three $(a, 4)=$ Three (AVAILPAR3 $+c 11,1)$

Three $(a+1,4)=$ Three $($ AVAILPAR3 $+c 11,1) ;$ Three $($ AVAILPAR3 $+c 11,3)=2 ;$

$\mathrm{c} 11=\mathrm{c} 11+1 ; \mathrm{a}=\mathrm{a}+2$;

end

AVAILOFF3S $=$ AVAILPAR3 $+(x 6 / 2)+1$

AVAILOFF3E = LAST_ROW_OF_MATRIX_THREE

num $=x 6-$ RANGE3; clear a;

pOS1 = AVAILOFF3S; pOS2 = AVAILOFF3S + num $-1 ; a=$ pos $1 ;$

while a $<$ pos 2

Three $(a, 4)=$ Three (AVAILPAR3 $+c 11,1)$;

Three $(a+1,4)=$ Three $($ AVAILPAR3 $+\mathrm{c} 11,1) ;$ Three $($ AVAILPAR3 $+\mathrm{c} 11,3)=2$;

$\mathrm{c} 11=\mathrm{c} 11+1 ; \mathrm{a}=\mathrm{a}+2$;

end

clear a;

else

pos1 = AVAILOFF3S; pos2 = AVAILOFF3S + RANGE3 -2; $\mathrm{C}=0$;

for $a=\operatorname{pos} 1:$ pos 2

$\mathrm{C}=\mathrm{C}+1$;

Three $(a, 4)=$ Three $($ AVAILPAR3 $+c 11,1) ;$ Three $($ AVAILPAR3 $+c 11,3)=c$;

if Three (AVAILPAR3 $+\mathrm{c} 11,3)==2$

$$
\mathrm{C} 11=\mathrm{C} 11+1 ; \mathrm{c}=0 \text {; }
$$

end

AVAILOFF3S = AVAILPAR3 + (x6/2) +1;

AVAILOFF 3E = LAST_ROW OF_MATRIX_THREE;

num $=\mathrm{x} 6-$ RANGE3; clear a

pos1 = AVAILOFF3S; pos2 = AVALLOFF3S + num $-1 ;$

for $a=$ pos $1:$ pos 2

$\mathrm{C}=\mathrm{C}+1$

Three $(a, 4)=$ Three $($ AVAILPAR3 $+c 11,1)$; Three $($ AVAILPAR3 $+c 11,3)=c$; if Three (AVAILPAR3 $+\mathrm{C} 11,3)==2$

$\mathrm{C} 11=\mathrm{C} 11+1 ; \quad \mathrm{C}=0$;

end

end

end 
end

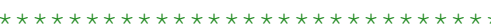

ㅇ. ASIGN PARENT ORDER 4 BRANCHES

if $\mathrm{y} 1 \sim=0$ \% checks to see whether there are any 4-1-1 branch types $\mathrm{C} 12=1 ;$ pos 1 = AVAILOFF1S; pos $2=$ AVALLOFF1S +y1 -2;

for $a=\operatorname{pos} 1: 2:$ pos $2 \%$

One $(a, 4)=$ Four $($ AVAILPAR $4+\mathrm{c} 12,1) ;$

One $(a+1,4)=$ Four (AVAILPAR4+c12,1);Four (AVAILPAR4 $+\mathrm{c} 12,3)=2 ; \circ$

$\mathrm{c} 12=\mathrm{c} 12+1$;

end

AVAILOFF $1 S=$ AVAILOFF $1 S+\mathrm{y} 1 ;$

AVAILPAR $4=$ AVAILPAR $4+\mathrm{c} 12-1$

AVAILOFF 4E= AVAILPAR 4;

RANGE $4=$ AVAILOFF 4E - AVAILOFF4S;

clear a;

end

if y2 =0 긍 checks to see whether there are any 4-1-2 branch types $\mathrm{c} 13=1 ;$ pos1 = AVAILOFF1S; pos2 = AVAILOFF1S + y2 -1;

for $a=\operatorname{pos} 1: \operatorname{pos} 2$ :

One $(a, 4)=$ Four $($ AVAILPAR $4+\mathrm{c} 13,1) ; \operatorname{Four}(\operatorname{AVAILPAR} 4+\mathrm{c} 13,3)=1$; $\mathrm{c} 13=\mathrm{c} 13+1 ;$

end

AVAILOFF1S $=$ pOS2 +1.

clear a; $\mathrm{c} 13=1 ;$ num $=\mathrm{y}^{2}+1$;

for $a=1$ : LAST_ROW_OF_MATRIX_TWO

if $\operatorname{Two}(a, 4)==0 \& \& \quad c 13<\operatorname{num}$

Two $(a, 4)=$ Four $($ AVAILPAR $4+\mathrm{c} 13,1) ;$ Four $($ AVAILPAR4 $+\mathrm{c} 13,3)=2$;

$\mathrm{c} 13=\mathrm{c} 13+1 ;$

end

end

AVAILPAR4 = AVAILPAR4 + c13-1;

AVAILNEW4 = AVAILPAR4 +1; clear a

AVAILOFF 4E = AVAILPAR4;

RANGE $4=$ AVAILOFF4E - AVAILOFF $4 S+1$ 
if $\mathrm{y} 3 \sim=0 \%$ checks to see whether there are any $4-1-3$ branch types $\mathrm{C} 14=1 ;$ pos $1=$ AVAILOFF $1 \mathrm{~S} ;$ pos $2=$ AVAILOFF1S $+\mathrm{y}^{3}-1 ;$ for $a=$ pos $1:$ pos $2 \%$

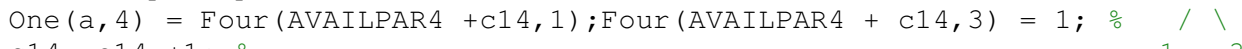

$\mathrm{c} 14=\mathrm{c} 14+1$;

end

AVAILOFF1S = AVAILOFF1S + y3

clear a; c14=1; num = y3 +1 ;

for $a=1$ : LAST_ROW_OF_MATRIX_THREE

if $\operatorname{Three}(a, 4)=0$ \&\& $\mathrm{c} 14<$ num

Three $(a, 4)=$ Four (AVAILPAR4 $+\mathrm{c} 14,1) ;$ Four (AVAILPAR4 $+\mathrm{c} 14,3)=2$;

$\mathrm{c} 14=\mathrm{c} 14+1$;

else

end

end

AVAILPAR4 = AVAILPAR4 + c14-1; clear a

AVAILOFF4E = AVAILPAR4;

RANGE $4=$ AVAILOFF4E - AVAILOFF4S +1;

end

if $\mathrm{y} 4 \sim=0 \%$ checks to see whether there are any $4-2-2$ branch types $\mathrm{c} 15=1 ; \operatorname{num}=(\mathrm{y} 4 / 2)+1 ; \mathrm{C}=0 ; \quad \circ$

for $a=1$ : LAST_ROW_OF_MATRIX_TWO

if $\operatorname{TwO}(a, 4)=0 \quad \& \& \quad c 15<$ num

$\mathrm{C}=\mathrm{C}+1$

Two $(a, 4)=$ Four $($ AVAILPAR4 $+\mathrm{c} 15,1) ;$ Four $($ AVAILPAR $4+\mathrm{c} 15,3)=\mathrm{c}$;

if Four (AVAILPAR4 $+\mathrm{c} 15,3)==2$

$\mathrm{c} 15=\mathrm{c} 15+1 ; \mathrm{c}=0$

end

end

end

AVAILPAR4 = AVAILPAR4 +C15 -1

AVAILOFF4E = AVAILPAR4;

AVAILNEW4 = AVAILPAR4 +1; clear $a$;

RANGE $4=$ AVAILOFF 4E - AVAILOFF 4S +1;

end 
if $\mathrm{y} 5 \sim=0 \div$ checks to see whether there are any 4-2-3 branch types $\mathrm{c} 16=1$; num = y5 +1;

for a $=1:$ I

if $\operatorname{Two}(a, 4)==\overline{0} \& \& \quad \mathrm{c} 16<$ num

Two $(a, 4)=$ Four $(\operatorname{AVAILPAR} 4+\mathrm{c} 16,1) ;$ Four $(\operatorname{AVAILPAR} 4+\mathrm{c} 16,3)=1 ; \% 23$

$\mathrm{c} 16=\mathrm{c} 16+1$;

end

clear a; $c 16=1 ;$ num $=$ y5 +1 ;

for $a=1$ : LAST_ROW_OF_MATRIX_THREE

if Three $(a, 4)=0$ \&\& $c 16<$ num

Three $(a, 4)=$ Four (AVAILPAR4 $+c 16,1) ;$ Four (AVAILPAR4 $+c 16,3)=2$; $\mathrm{c} 16=\mathrm{c} 16+1$;

end

end

AVAILPAR4 = AVAILPAR $4+c 16-1$

AVAILNEW4 = AVAILPAR4 +1; Clear

AVAILOFF 4E = AVAILPAR 4

RANGE $4=$ AVAILOFF4E - AVAILOFF4S +1;

end

if $\mathrm{y} 6 \sim=0 \div$ checks to see whether there are any $4-3-3$ branch types $\mathrm{c} 17=1 ;$ num $=(\mathrm{y} 6 / 2)+1 ; \mathrm{c}=0$;

for $a=1:$ LAST_ROW_OF_MATRIX_THREE

if $\operatorname{Three}(a, 4)=0$ \&\& $\quad 17<$ num $\frac{0}{0}$

$\mathrm{C}=\mathrm{C}+1 ; \circ$

Three $(a, 4)=$ Four (AVAILPAR4 $+c 17,1) ;$ Four (AVAILPAR4 $+c 17,3)=c ; \%$

if Four (AVAILPAR4 + c17, 3) $==2$

$\mathrm{C} 17=\mathrm{c} 17+1 ; \mathrm{C}=0$

end

end

end

AVAILPAR $4=$ AVAILPAR $4+C 17-1$

AVAILOFF 4E = AVAILPAR 4;

AVAILNEW4 = AVAILPAR4 +1; clear $a_{i}$

RANGE4 = AVAILOFF4E - AVAILOFF 4S +1;

clear a;

end 
if $\mathrm{y} 7 \sim=0 \%$ checks to see whether there are any 4-1-4 branch types check = AVAILPAR $4+1$;

if AVATLOFF4S $=1$ \& 1 check $==1$ \%

AVAILPAR4 = y7; AVAILOFF 4E = y7; RANGE4 = AVAILOFF4E-AVALLOFF 4S +1;

end $\%$

$\mathrm{C} 18=1 ; \operatorname{pos} 1=$ AVALLOFF1S; pos $2=\operatorname{AVAILOFF} 1 \mathrm{~S}+\mathrm{y} 7-1 ; \%$

for $a=$ posi:pos2

One $(a, 4)=$ Four $($ AVAILPAR $4+\mathrm{c} 18,1) ;$ Four $($ AVAILPAR $4+\mathrm{c} 18,3)=1$

$\mathrm{c} 18=\mathrm{c} 18+1$;

end

AVAILOFF $1 \mathrm{~S}=$ pos $2+1$

clear a; $\mathrm{c} 18=1$;

if $\mathrm{y} 7<$ RANGE $4 \%$ checks to see whether range of available offspring is greater than the reguired number of offspring

pos1 = AVAILOFF4S; pos2 = AVAILOFF4S +y7 -1;

for a $=$ pos $1:$ pos 2

Four $(a, 4)=$ Four (AVAILPAR4 $+\mathrm{c} 18,1) ;$ Four (AVAILPAR $4+c 18,3)=2$

$\mathrm{c} 18=\mathrm{c} 18+1$

end

AVAILOFF 4 S $=$ pOS $2+1$

AVAILPAR4 = AVAILPAR4 + c18 -1; AVAILOFF4E = AVAILPAR4;

RANGE $4=$ AVAILOFF4E - AVAILOFF 4 S + 1

clear a

else

if $\mathrm{y} 7==$ RANGE $4 \%$ checks to see whether range of available offspring is equal to the required number of offspring

pos1 = AVAILOFF4S; pos2 = AVAILOFF4S + RANGE4 -1

for $a=$ pos $1:$ pos 2

Four $(a, 4)=$ Four $($ AVAILPAR $4+\mathrm{c} 18,1) ;$ Four $(\operatorname{AVAILPAR} 4+\mathrm{c} 18,3)=2$

$\mathrm{c} 18=\mathrm{c} 18+1$

end

off $=$ pos $2+1$

while Four (off, 4) $\sim=0$

off $=$ off +1

end

AVAILOFF4S = Off; AVAILOFF4E = AVAILPAR4 + $18-1 ;$

AVAILPAR4 $=$ AVAILPAR4 + $18-1 ;$

RANGE 4 = AVAILOFF4E - AVAILOFF 4 S +1 
if $\mathrm{y} 7>$ RANGE $4 \%$ checks to see whether range of available offspring is less than the required number of offspring

pos1 = AVAILOFF4S; pos2 = AVAILOFF4S + RANGE4 -1;

for $a=$ posi:pos2

Four $(a, 4)=$ Four (AVAILPAR4 $+\mathrm{c} 18,1) ;$ Four $($ AVAILPAR4 $+\mathrm{c} 18,3)=2$;

$\mathrm{c} 18=\mathrm{c} 18+1$

end

AVAILOFF4S $=$ AVAILPAR $4+\mathrm{y} 7+1 ;$

AVAILOFF 4E = LAST_ROW_OF_MATRIX_FOUR;

num $=$ y 7 - RANGE4; clear a;

pOS1 = AVAILOFF4S; POS2 = AVAILOFF4S + num -1i

for $a=$ posi:pos 2

Four $(a, 4)=$ Four (AVAILPAR $4+\mathrm{c} 18,1) ;$ Four $($ AVAILPAR $4+\mathrm{c} 18,3)=2$;

$\mathrm{c} 18=\mathrm{c} 18+1$

end

AVAILOFF4S $=$ AVAILPAR $4+1$

AVAILPAR4 = AVAILPAR $4+\mathrm{C} 18-1$;

Clear a; AVAILOFF4E = AVAILPAR4;

RANGE $4=$ AVAILOFF4E - AVAILOFF $4 S+1$;

end

end

end

end

if $\mathrm{y} 8 \sim=0 \div$ checks to see whether there are any 4-2-4 branch types check = AVAILPAR $4+1$;

if AVAILOFF4S $==1$ \& $\&$ Check $==1 \%$

AVAILPAR4 = y8; AVAILOFF4E = y8; RANGE4 = AVAILOFF4E-AVAILOFF4S +1; $/$

end $\frac{\circ}{\circ}$

$\mathrm{C} 19=1 ;$ num $=\mathrm{y} 8+1$;

for $a=1$ : LAST_ROW_OF_MATRIX_TWO

if $\operatorname{Two}(a, 4)==0 \quad \& \& \quad c 19<$ num

$\operatorname{Two}(a, 4)=$ Four (AVAILPAR4 + c19, 1) ; Four (AVAILPAR4 +c19, 3) $=1 ;$

$\mathrm{c} 19=\mathrm{c} 19+1$;

end

end

clear a; $\mathrm{c} 19=1 ;$ num $=\mathrm{y} 8+1$ 
if $\mathrm{y} 8<\operatorname{RANGE} 4 \% \mathrm{checks}$ to see whether range of available offspring is greater than the required number of offspring

pos1 = AVAILOFF4S; pos2 = AVAILOFF 4S +y8 -1;

for $a=$ posi:pos2

Four $(a, 4)=$ Four $($ AVAILPAR4 $+c 19,1) ;$ Four $($ AVAILPAR4 $+c 19,3)=2$;

c19 = c19+1;

end

AVAILOFF4S $=$ POS2 +1

AVAILPAR4 $=$ AVAILPAR $4+\mathrm{C} 19-1 ;$ AVAILOFF4E $=$ AVAILPAR4

RANGE $4=$ AVAILOFF4E - AVAILOFF 4 S +1;

clear a

else

if $\mathrm{y} 8==$ RANGE $4 \%$ checks to see whether range of available offspring is equal to the reguired number of offspring

pos1 = AVALLOFF4S; pos2 = AVAILOFF4S + RANGE4 -1;

for $a=$ pos $1:$ pos 2

Four $(a, 4)=$ Four (AVAILPAR $4+c 19,1) ;$ Four (AVAILPAR4 $+c 19,3)=2 ;$

c19 $=\mathrm{c} 19+1$

end

off $=$ pos $2+1$

while Four (off, 4$) \sim=0$

off $=$ off +1 ;

end

AVAILOFF4S = Off; AVAILOFF4E = AVAILPAR4 + C19 - 1

AVAILPAR4 = AVAILPAR4 + C19 -1;

RANGE $4=$ AVAILOFF 4E - AVALLOFF $4 \mathrm{~S}+1$;

else

if y8 > RANGE $4 \%$ checks to see whether range of available offspring is less than the required number of offspring

pos1 = AVALLOFF4S; pos2 = AVAILOFF4S + RANGE4 -1;

for $\mathrm{a}=\operatorname{pos} 1: \operatorname{pos} 2$

Four $(a, 4)=$ Four (AVAILPAR $4+c 19,1) ;$ Four (AVAILPAR $4+c 19,3)=2$

$\mathrm{c} 19=\mathrm{c} 19+1$

end

AVAILOFF4S = AVAILPAR4 + y8 +1; AVAILOFF4E = LAST_ROW_OF_MATRIX_FOUR

num $=$ y8 - RANGE4; clear a;

pOS1 = AVAILOFF4S; pos2 = AVAILOFF4S + num $-1 ;$

for $a=$ posi:pos2 
Four $(a, 4)=$ Four (AVAILPAR4 $+\mathrm{c} 19,1) ;$ Four $($ AVAILPAR4 $+\mathrm{c} 19,3)=2$;

$\mathrm{c} 19=\mathrm{c} 19+1$

end

AVAILOFF 4 S $=$ AVAILPAR $4+1$

AVAILPAR4 = AVAILPAR4 + $19-1$;

clear a; AVAILOFF4E = AVAILPAR4

RANGE $4=$ AVAILOFF4E - AVAILOFF $4 \mathrm{~S}+1 i$

end

end

end

end

if $\mathrm{y} 9 \sim=0$ 을 check = AVAILPAR4 + 1 ;

if AVAILOFF $4 S==1$ \&\& check $==1$ 응

AVAILPAR4 = y9; AVAILOFF4E = y9; RANGE4 = AVAILOFF4E-AVAILOFF4S + 1 ;

end응

check = AVAILPAR4 + 1

c20=1; num = y $9+1$;

for $a=1$ : LAST_ROW_OF_MATRIX_THREE

if $\operatorname{Three}(a, 4)==0$ \&\& $\quad 20<$ num

Three $(a, 4)=$ Four $($ AVAILPAR $4+c 20,1) ;$ Four $($ AVAILPAR $4+c 20,3)=1$;

$\mathrm{c} 20=\mathrm{c} 20+1$;

end

end

clear a; c20=1; num = y9+1;

if $\mathrm{y} 9<\mathrm{RANGE} 4 \% \mathrm{checks}$ to see whether range of available offspring is greater than the required number of offspring

pos1 = AVAILOFF4S; pos2 = AVALLOFF4S +y9 -1;

for a $=$ pos $1:$ pos 2

Four $(a, 4)=$ Four (AVAILPAR $4+c 20,1) ;$ Four $($ AVAILPAR4 $+c 20,3)=2$;

$\mathrm{c} 20=\mathrm{c} 20+1$

end

AVAILOFF4S $=$ pOS $2+1$

AVAILPAR4 = AVAILPAR4 + C20 -1;AVAILOFF4E = AVAILPAR 4

RANGE 4 = AVAILOFF4E - AVAILOFF $4 \mathrm{~S}+1$;

clear a;

else 
if $\mathrm{y} 9==$ RANGE $4 \%$ checks to see whether range of available offspring is equal to the required number of offspring

pos1 = AVAILOFF4S; pos2 = AVAILOFF4S + RANGE $4-1 ;$

for $a=\operatorname{pos} 1:$ pos2

Four $(a, 4)=$ Four $(\operatorname{AVAILPAR} 4+\mathrm{c} 20,1) ;$ Four $(\operatorname{AVAILPAR} 4+\mathrm{c} 20,3)=2$

$\mathrm{c} 20=\mathrm{c} 20+1$

end

off $=$ pos $2+1$

while Four (off, 4) $\sim=0$

off $=$ off +1

end

AVAILOFF4S = off; AVAILOFF4E = AVAILPAR4 + $20-1 ;$

AVAILPAR4 $=$ AVAILPAR $4+\mathrm{C} 20-1$;

RANGE $4=$ AVAILOFF4E - AVAILOFF $4 \mathrm{~S}+1$

else

if $\mathrm{y}^{9}>$ RANGE $4 \%$ checks to see whether range of available offspring is less than the required number of offspring

pos1 = AVALLOFF4S; pos2 = AVAILOFF4S + RANGE4 -1;

for $a=$ posi:pos 2

Four $(a, 4)=$ Four $($ AVAILPAR $4+c 20,1) ;$ Four $($ AVAILPAR $4+c 20,3)=2$;

$\mathrm{c} 20=\mathrm{c} 20+1 ;$

end

AVAILOFF4S = AVAILPAR4 + Y9 +1; AVAILOFF4E = LAST_ROW_OF MATRIX_FOUR

num $=$ y9 - RANGE4; clear $a$;

pos1 = AVAILOFF4S; pos2 = AVAILOFF 4 S + num -1

for $a=$ posi:pos2

Four $(a, 4)=$ Four $($ AVAILPAR $4+c 20,1) ;$ Four $($ AVAILPAR4 $+c 20,3)=2$;

$\mathrm{C} 20=\mathrm{C} 20+1$

end

AVAILOFF4S = AVAILPAR4 + 1 ;

AVAILPAR $4=$ AVAILPAR $4+\mathrm{C} 20-1 ;$

AVAILNEW4 = pos2 +1; clear a; AVAILOFF4E = AVAILPAR4

RANGE 4 = AVAILOFF 4E - AVAILOFF $4 \mathrm{~S}+1 ;$

end

end

end

end

if $\mathrm{y} 10 \sim=0 \%$ checks to see whether there are any 4-4-4 branch types 
check = AVAILPAR4 $+1 ; \%$

if AVALLOFF4S $=1$ \&\& Check $==1 \%$

AVAILPAR4 = y10; AVAILOFF4E = y10; RANGE4 = AVALLOFF4E-AVAILOFF $4 \mathrm{~S}+1 ;$

end응

c $21=1$

if y $10<$ RANGE $4 \%$ checks to see whether range of available offspring is greater than the required number of offspring

pos1 = AVALLOFF4S; pos2 = AVALLOFF4S + y10 -1; $\mathrm{a}=$ pos1;

while a pos2

Four $(a, 4)=$ Four $($ AVAILPAR $4+c 21,1)$

Four $(a+1,4)=$ Four (AVAILPAR $4+\mathrm{c} 21,1) ;$ Four (AVAILPAR4 $+\mathrm{c} 21,3)=2$;

$c 21=c 21+1 ; a=a+2$;

end

AVAILOFF $4 S=$ pOs $2+1 ;$

AVAILPAR4 = AVAILPAR4 + c21 -1;AVAILOFF4E = AVAILPAR4;

RANGE $4=$ AVAILOFF4E - AVAILOFF $4 S+1 ;$

clear a;

else

if $\mathrm{y} 10==$ RANGE $4 \%$ checks to see whether range of available offspring is equal to the required number of offspring

pos1 = AVAILOFF4s; pos2 = AVAILOFF4s + RANGE4 -1; a = pos1;

while a $<$ pos2

Four $(a, 4)=$ Four (AVAILPAR4 $+\mathrm{c} 21,1)$;

Four $(a+1,4)=$ Four $($ AVAILPAR $4+\mathrm{c} 21,1) ;$ Four $(\operatorname{AVAILPAR} 4+\mathrm{c} 21,3)=2$;

$\mathrm{c} 21=\mathrm{c} 21+1 ; \mathrm{a}=\mathrm{a}+2$;

end

off $=$ pos $2+1$

while Four (off, 4) $\sim=0$

off $=$ off +1 ;

end

AVAILOFF4S = Off; AVAILOFF4E = AVAILPAR4 + C21-1;

AVAILPAR4 = AVAILPAR4 + $\mathrm{c} 21-1 ; \mathrm{c} 21=1 ;$ clear $a ;$

RANGE4 = AVAILOFF4E - AVAILOFF4S +1

else

if y10>RANGE $4 \%$ checks to see whether range of available offspring is less than the required number of offspring

c21=1; if rem(RANGE 4,2$)==0$ 
pos1 = AVAILOFF4S; pos2 = AVAILOFF 4S + RANGE4 $-1 ; a=$ pos1;

while a pos2

Four $(a, 4)=$ Four (AVAILPAR4 + c21, 1);

Four $(a+1,4)=$ Four (AVAILPAR $4+c 21,1) ;$ Four (AVAILPAR4 + c21, 3) = 2;

$\mathrm{c} 21=\mathrm{c} 21+1 ; \mathrm{a}=\mathrm{a}+2$;

end

AVAILOFF4S = AVAILPAR4 + (y10/2) +1;

AVAILOFF $4 E=$ LAST_ROW_OF_MATRIX_FOUR

num $=$ y10 - RANGE 4; clear a;

pos1 = AVALLOFF4S; pos2 = AVALLOFF4S + num $-1 ; \mathrm{a}=$ pos1;

while a pos2

Four $(a, 4)=$ Four (AVAILPAR $4+c 21,1)$

Four $(a+1,4)=$ Four (AVAILPAR4 $+\mathrm{c} 21,1) ;$ Four (AVAILPAR4 $+\mathrm{c} 21,3)=2$;

$c 21=c 21+1 ; a=a+2 ;$

end

clear $a$;

else

pos1 = AVALLOFF4S; pos2 = AVAILOFF4S + RANGE4 -1;

for $\mathrm{a}=$ pos $1:$ pos 2

$\mathrm{C}=\mathrm{C}+1$;

Four $(a, 4)=$ Four $($ AVAILPAR4 $+c 21,1) ;$ Four $($ AVAILPAR4 $+c 21,3)=c$;

if Four (AVAILPAR $4+c 21,3)==2$

end

end

AVAILOFF $4 S=$ AVAILPAR $4+(y 10 / 2)+1$

AVAILOFF 4E = LAST_ROW_OF_MATRIX_FOUR;

num $=$ y10 - RANGE 4; clear a;

pos1 = AVALLOFF4S; pos2 = AVAILOFF4S + num -1

for $a=\operatorname{pos} 1: \operatorname{pos} 2$

Four $(a, 4)=$ Four $($ AVAILPAR $4+c 21,1) ;$ Four $($ AVAILPAR $4+c 21,3)=c$;

if Four (AVAILPAR $4+\mathrm{c} 21,3)==2$

end $\mathrm{C} 21=\mathrm{C} 21+1 ; \mathrm{C}=0$;

end

clear a;

end

end

end

end 
if $\mathrm{z} 1 \sim=0 \%$ checks to see whether there are any 5-2-2 branch types $\mathrm{c} 22=1 ; \operatorname{num}=(\mathrm{z} 1 / 2)+1 ; \mathrm{c}=0$;

for $a=1$ : LAST_ROW_OF_MATRIX_TWO

if $\operatorname{TwO}(a, 4)=0$ \&\& $c 22<$ num

$\mathrm{C}=\mathrm{C}+1 ;$ \%

Two $(a, 4)=$ Five (AVAILPAR5 + c22, 1$)$; Five (AVAILPAR5 + c22,3) = c;

if Five(AVAILPAR5 $+\mathrm{c} 22,3)==2$

$\mathrm{c} 22=\mathrm{c} 22+1 ; \mathrm{c}=0$;

end

end

end

AVAILPAR5 = AVAILPAR5 +C22 -1; AVAILOFF5E = AVAILPAR5;

clear a; RANGE5 = AVAILOFF5E - AVAILOFF5S +1;

end

if $z 2 \sim=0 \div$ checks to see whether there are any 5-2-3 branch types $\mathrm{c} 23=1$; num = z2 +1;

for $a=1$ : LAST ROW OF MATRIX TWO

if $\operatorname{TwO}(a, 4)==\overline{0} \& \& \quad$ C $23<$ num $\%$

$\operatorname{Two}(a, 4)=$ Five $($ AVAILPAR5 $+\mathrm{c} 23,1) ;$ Five $(\operatorname{AVAILPAR} 5+\mathrm{c} 23,3)=1$; $\%$

$\mathrm{c} 23=\mathrm{c} 23+1 ;$

end

clear $a ; c 23=1 ;$ num $=z 2+1 ;$

for $a=1$ : LAST_ROW_OF_MATRIX_THREE

if Three $(a, 4)=0$ \&\& $c 23<$ num

Three $(a, 4)=$ Five (AVAILPAR5 $+c 23,1)$; Five (AVAILPAR5 $+c 23,3)=2$

$c 23=c 23+1 ;$

end

end

AVAILPAR5 = AVAILPAR5 + $23-1$;

clear a; AVAILOFF5E = AVAILPAR5; 
RANGE5 = AVAILOFF5E - AVALLOFF5S +1;

end

if $z 3 \sim=0 \div$ checks to see whether there are any 5-2-4 branch types $\mathrm{c} 24=1 ;$ num $=\mathrm{z} 3+1$;

for $a=1$ : LAST_ROW_OF_MATRIX_TWO

if $\operatorname{Two}(a, 4)==0 \& \& \quad c 24<$ num $\%$

$\operatorname{Two}(a, 4)=$ Five $($ AVAILPAR5 $+c 24,1) ;$ Five $(\operatorname{AVAILPAR} 5+c 24,3)=1$;

$\mathrm{c} 24=\mathrm{c} 24+1$;

end

end

clear a; $c 24=1 ;$ num $=z 3+1 ;$

for $a=1$ : LAST_ROW_OF_MATRIX_FOUR

if $\operatorname{Four}(a, 4)==0$ \&\& $C 24<$ num

Four $(a, 4)=$ Five (AVAILPAR5 + c24, 1); Five (AVAILPAR5 + c24,3) = 1;

$\mathrm{c} 24=\mathrm{c} 24+1$;

end

end

AVAILPAR5 = AVAILPAR5 + C24-1;

clear a; AVAILOFF5E = AVAILPAR5

RANGE5 = AVAILOFF5E - AVAILOFF5S +1;

end

if $z 4 \sim=0$ \% checks to see whether there are any 5-3-3 branch types $\mathrm{c} 25=1 ;$ num $=(\mathrm{z} 4 / 2)+1 ; \mathrm{c}=0$;

for $a=1$ :LAST ROW OF MATRIX THREE

if Three $(a, 4)=0$ \&\& $\quad 25<$ num $\frac{\circ}{0}$

$\mathrm{C}=\mathrm{C}+1 ;$ \%

Three $(a, 4)=$ Five $($ AVAILPAR5 + c25, 1); Five $($ AVAILPAR5 + c25,3) = c ;

if Five(AVAILPAR5 +c25, 3) ==

$\mathrm{c} 25=\mathrm{c} 25+1 ; \mathrm{c}=0$;

end

end

AVAILPAR5 = AVAILPAR5 +C25 -1; AVAILOFF5E = AVAILPAR5;

clear a; RANGE5 = AVAILOFF5E - AVAILOFF5S +1;

end

if $\mathrm{z} 5 \sim=0$ 으ecks to see whether there are any 5-3-4 branch types 
$\mathrm{c} 26=1 ;$ num $=\mathrm{z} 5+1$;

for $a=1$ : LAST_ROW_OF_MATRIX_THREE $\%$

if Three $(a, 4)==0$ \&\& $\quad 26<$ num $\frac{\circ}{0}$

Three $(a, 4)=$ Five $($ AVAILPAR5 $+\mathrm{c} 26,1)$; Five $($ AVAILPAR5 $+\mathrm{c} 26,3)=1$;

$\mathrm{c} 26=\mathrm{c} 26+1 ;$

end

end

clear a;

c26=1; num $=25+1$;

for $a=1$ : LAST_ROW_OF_MATRIX_FOUR

if $\operatorname{Four}(a, 4)=0$ \&\& $c 26<$ num

Four $(a, 4)=$ Five (AVAILPAR5 $+c 26,1) ;$ Five $($ AVAILPAR5 $+c 26,3)=2$; $c 26=c 26+1 ;$

end

end

AVAILPAR5 = AVAILPAR5 + $26-1 ;$

Clear a; AVAILOFF5E = AVAILPAR5

RANGE5 = AVAILOFF5E - AVAILOFF5S +1;

end

if $\mathrm{z} 6 \sim=0$ \% checks to see whether there are any 5-4-4 branch types $\mathrm{c} 27=1 ;$ num $=(\mathrm{z} 6 / 2)+1 ; \quad \mathrm{c}=0$;

for $a=1$ :LAST_ROW_OF_MATRIX_FOUR

if $\operatorname{Four}(\mathrm{a}, 4)=0$ \& $\mathrm{\varepsilon}$ c $27<$ num\%

if Four $(a, 4$
$C=C+1 ; \%$

Four $(a, 4)=$ Five $($ AVAILPAR5 + c27, 1$) ;$ Five $($ AVAILPAR5 + c27, 3) $=c$; $\%$

if Five(AVAILPAR5 $+\mathrm{c} 27,3)==2$

$\mathrm{c} 27=\mathrm{c} 27+1 ; \mathrm{c}=0$;

end

end

end

AVAILPAR5 = AVAILPAR5 +C27 -1; AVAILOFF5E = AVAILPAR5;

clear a; RANGE5 = AVAILOFF5E - AVAILOFF5S +1;

end

if $\mathrm{z} 7 \sim=0$ 을 checks to see whether there are any 5-2-5 branch types check = AVAILPAR5 $+1 ;$

if AVAILOFF5S $==1$ \&\& check $==1$ \%

AVAILPAR5 $=z 7 ;$ AVAILOFF5E $=z 7 ;$ RANGE5 = AVAILOFF5E-AVAILOFF5S $+1 ; \circ$ 
end $\%$

$\mathrm{c} 28=1 ; \mathrm{num}=\mathrm{z} 7+1 ;$

for $a=1$ : LAST ROW_OF_MATRIX_TWO

if $\operatorname{TwO}(a, 4)==\overline{0} \& \& \quad \mathrm{C} 28<$ num

Two $(a, 4)=$ Five $($ AVAILPAR5 + c28, 1$)$; Five $($ AVAILPAR5 + c28,3) $=1$;

$\mathrm{c} 28=\mathrm{c} 28+1$

end

clear a; $c 28=1$;

if $\mathrm{z} 7<$ RANGE $5 \%$ checks to see whether range of available offspring is greater than the required number of offspring

pos1 = AVAILOFF5S; pos2 = AVALLOFF5S + z7 -1;

for $a=$ posi:pos 2

Five $(a, 4)=$ Five $($ AVAILPAR5 $+\mathrm{c} 28,1) ;$ Five $($ AVAILPAR5 $+\mathrm{c} 28,3)=2$

end

AVAILOFF5S $=$ pOS $2+1$

AVAILPAR5 = AVAILPAR5 + C28 -1; AVAILOFF5E = AVAILPAR5

RANGE5 = AVAILOFF5E - AVAILOFF5S + 1

clear a;

else

if $\mathrm{z} 7==$ RANGE5\%checks to see whether range of available offspring is equal to the required number of offspring

pos1 = AVAILOFF5S; pos2 = AVAILOFF5S + RANGE5 -

for $a=$ posi:pos2

Five $(a, 4)=$ Five $($ AVAILPAR5 $+\mathrm{c} 28,1) ;$ Five $($ AVAILPAR5 $+\mathrm{c} 28,3)=2$

$\mathrm{c} 28=\mathrm{c} 28+1$

off $=$ pos $2+1$

while Five $($ off, 4$) \sim=0$

off $=$ off +1

end

AVAILOFF5S = off; AVAILOFF5E = AVAILPAR5 + C28 - 1

AVAILPAR5 $=$ AVAILPAR $5+\mathrm{C} 28-1$

RANGE5 = AVAILOFF5E - AVAILOFF5S +1;

else 
if $\mathrm{z} 7>$ RANGE5\%checks to see whether range of available offspring is less than the required number of offspring

pos1 = AVAILOFF5S; pos2 = AVAILOFF5S + RANGE5 $-1 ;$

for $a=$ posi:pos2

Five $(a, 4)=$ Five (AVAlLPAR5 + c28, 1); Five $($ AVAILPAR5 + c28,3) = 2 ;

$\mathrm{c} 28=\mathrm{c} 28+1$;

end

AVAILOFF5S = AVAILPAR5 $+\mathrm{z} 7+1 ;$ AVAILOFF5E = LAST_ROW_OF_MATRIX_FIVE

num $=\mathrm{z} 7$ - RANGE5; clear a

pos1 = AVAILOFF5S; pos $2=$ AVAILOFF5S + num -1

for a $=$ posi:pos2

Five $(a, 4)=$ Five $($ AVAILPAR5 $+\mathrm{c} 28,1) ;$ Five $($ AVAILPAR5 $+\mathrm{c} 28,3)=2$;

$\mathrm{C} 28=\mathrm{C} 28+1$

AVAILOFF5S $=$ AVAILPAR5 +1

AVAILPAR5 = AVAILPAR5 + C28-1;

AVAILNEW5 = pos2 +1 ; clear a; AVAILOFF5E = AVAILPAR5;

RANGE5 = AVAILOFF5E - AVAILOFF5S +1;

end

end

end

end

if $\mathrm{z} 8 \sim=0$ 을 checks to see whether there are any 5-3-5 branch types

check = AVAILPAR5 +1

if AVAILOFF5S $==1$ \&\& check $==1$

AVAILPAR5 $=z 8 ;$ AVAILOFF5E = z8; RANGE5 = AVALLOFF5E-AVAILOFF5 $+1 ;$ 응 end \%

c29=1; num = z8 +1; \%

for $a=1$ : LAST_ROW_OF_MATRIX_THREE

if Three $(a, 4)=0$ \&\& $c 29<$ num

Three $(a, 4)=$ Five (AVAILPAR5 + c29, 1); Five $($ AVAILPAR5 + c29, 3) = 1 ;

c29 = c29+1;

end

clear a; c29=1;

if $z 8<$ RANGE $5 \%$ checks to see whether range of available offspring is greater than the required number of offspring

pos1 = AVAILOFF5S; pos2 = AVAILOFF5S +z8 -1; 
for $a=$ posi:pos 2

Five $(a, 4)=$ Five $($ AVAILPAR5 + c29, 1$) ;$ Five $($ AVAILPAR5 + c29, 3) $=2$;

$\mathrm{c} 29=\mathrm{c} 29+1$

end

AVAILOFF5S $=$ pOS2 +1 ;

AVAILPAR5 = AVAILPAR5 + c29 -1; AVAILOFF5E = AVAILPAR5;

RANGE5 = AVAILOFF5E - AVAILOFF5S + $i$

AVAILNEW5 = AVAILPAR5 + 1 ;

clear a;

else

if $\mathrm{z} 8==$ RANGE $5 \%$ checks to see whether range of available offspring is equal to the required number of offspring

pos $1=$ AVALLOFF5S; pos2 = AVALLOFF5S + RANGE5 -1 ;

for $a=$ pos $1:$ pos 2

Five $(a, 4)=$ Five $($ AVAILPAR5 + c29,1); Five $($ AVAILPAR5 + c29, 3) $=2$

$\mathrm{c} 29=\mathrm{c} 29+1$

end

off $=$ pos $2+1$

while Five $($ off, 4$) \sim=0$

off $=$ off +1 ;

end

AVAILOFF5S = off; AVAILOFF5E = AVAILPAR5 + c29-1;

AVAILPAR5 $=$ AVAILPAR5 + C29-1

RANGE5 = AVAILOFF5E - AVALLOFF5S +1;

else

if z8 > RANGE5\%checks to see whether range of available offspring is less than the required number of offspring

pos1 = AVALLOFF5S; pos2 = AVALLOFF5S + RANGE5 - 1 ;

for $a=$ posi:pos2

Five $(a, 4)=$ Five (AVAlLPAR5 + c29, 1); Five (AVAILPAR5 + c29,3) = 2 ;

$\mathrm{c} 29=\mathrm{c} 29+1 ;$

end

AVAILOFF5S = AVAILPAR5 + z8 +1; AVAILOFF5E = LAST ROW OF MATRIX_FIVE

num $=\mathrm{z} 8-$ RANGE5; clear a

pos1 = AVALLOFF5S; pos2 = AVALLOFF5S + num $-1 ;$

for $a=$ pos $1:$ pos 2

Five $(a, 4)=$ Five $($ AVAILPAR5 + c29, 1); Five (AVAILPAR5 + c29, 3) $=2$;

$\mathrm{c} 29=\mathrm{c} 29+1$ 
end

AVAILOFF5S $=$ AVAILPAR5 $+1 ;$

AVAILPAR5 = AVAILPAR5 + c29-1;

clear a; AVAILOFF5E = AVAILPAR5

RANGE5 = AVAILOFF5E - AVALLOFF5S +1;

end

end

end

end

if $z 9 \sim=0$ 응 checks to see whether there are any 5-4-5 branch types

check = AVAILPAR5 +1 ;

if AVAILOFF5S $==1$ \& $\&$ Check $==1 \%$

AVAILPAR $5=z 9 i$ AVATLOFF5E $=z 9 i$ RANGE $5=$ AVATLOEF5E-AVATLOFE5S $+1 ;$ end $\%$

$\mathrm{c} 30=1$; num $=\mathrm{z} 9+1$;

for $a=1$ : LAST_ROW_OF_MATRIX_FOUR

if $\operatorname{Four}(a, 4)==0$ \&\& $\quad \mathrm{c} 30<$ num

Four $(a, 4)=$ Five $($ AVAILPAR5 $+\mathrm{c} 30,1) ;$ Five $($ AVAILPAR5 $+\mathrm{c} 30,3)=1$;

c30 $=$ c30 +1;

end

end

clear a; c30=1;

if $\mathrm{z} 9<\mathrm{RANGE} 5 \%$ checks to see whether range of available offspring is greater than the required number of offspring

pos1 = AVAILOFF5S; pos2 = AVAILOFF5S +z9 -1;

for $a=$ posi:pos2

Five $(a, 4)=$ Five $($ AVAILPAR5 $+c 30,1) ;$ Five $($ AVAILPAR5 $+c 30,3)=2$;

$\mathrm{c} 30=\mathrm{c} 30+1$

end

AVAILOFF5S = pOS $2+1$

AVAILPAR5 $=$ AVAILPAR5 + C30 -1;AVAILOFF5E = AVAILPAR5

RANGE5 = AVAILOFF5E - AVAILOFF5S +1

clear a

else

if $\mathrm{z} 9==$ RANGE5\%checks to see whether range of available offspring is equal to the required number of offspring 
pos1 = AVAILOFF5S; pos2 = AVAILOFF5S + RANGE5 $-1 ;$

for $a=$ pos $1:$ pos 2

Five $(a, 4)=$ Five (AVAILPAR5 + $c 30,1) ;$ Five(AVAILPAR5 $+c 30,3)=2 i$

$\mathrm{c} 30=\mathrm{c} 30+1$

end

off $=$ pos $2+1$

while Five(off,4) $\sim=0$

off $=$ off +1 ;

end

AVAILOFF5S = off; AVAILOFF5E = AVAILPAR5 + C30 - 1

AVAILPAR5 = AVAILPAR5 + C30-1;

RANGE5 = AVAILOFF5E - AVAILOFF5S +1

else

if $\mathrm{z} 9>$ RANGE5\%checks to see whether range of available offspring is less than the required number of offspring

pos1 = AVAILOFF5S; pos2 = AVAILOFF5S + RANGE5 -1;

for $a=$ posi:pos 2

Five $(a, 4)=$ Five (AVAILPAR5 $+\mathrm{c} 30,1) ;$ Five $($ AVAILPAR5 $+c 30,3)=2$;

$\mathrm{c} 30=\mathrm{c} 30+1 ;$

end

AVAILOFF5S = AVAILPAR5 + z9 +1; AVAILOFF5E = LAST_ROW_OF_MATRIX_FIVE

num $=$ z9 - RANGE5; clear a;

pos1 = AVAILOFF5S; pos2 = AVAILOFF5S + num -1

for $\mathrm{a}=$ posi:pos2

Five $(a, 4)=$ Five $($ AVAILPAR5 $+\mathrm{c} 30,1) ;$ Five $($ AVAILPAR5 $+\mathrm{c} 30,3)=2$;

$\mathrm{c} 30=\mathrm{c} 30+1$

end

AVAILOFF5S = AVAILPAR5 + 1

AVAILPAR5 = AVAILPAR5 + $\mathrm{C} 30-1 ;$

AVAILNEW5 = pos2 +1; clear a; AVAILOFF5E = AVAILPAR5

RANGE5 = AVAILOFF5E - AVAILOFF $5 S+1$

end

end

end

end

if z10 =0 $\frac{\circ}{0}$ checks to see whether there are any 5-5-5 branch types check = AVAILPAR5 $+1 ;$ 
if AVAILOFF5S $==1 \& \&$ check $==1 \%$

AVAILPAR5 = z10; AVAILOFF5E = z10; RANGE5 = AVAILOFF5E-AVAILOFF5S $+1 ; \%$

end \%

$\mathrm{c} 31=1 ; ;$

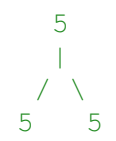

if z10<RANGE $5 \%$ checks to see whether range of available offspring is greater than the required number of offspring

pos1 = AVALLOFF5S; pos $2=$ AVALLOFF $5 S+z 10-1 ; a=$ pos 1

while a pos2

Five $(a, 4)=$ Five $($ AVAILPAR5 + c31, 1$)$;

Five $(a+1,4)=$ Five (AVAILPAR5 + c31,1); Five (AVAILPAR5 + c31, 3$)=2$

$\mathrm{c} 31=\mathrm{c} 31+1 ; \mathrm{a}=\mathrm{a}+2$;

end

AVAILOFF5S $=$ pOs $2+1$;

AVAILPAR5 = AVAILPAR5 + $31-1$; AVAILOFF5E = AVAILPAR5;

RANGE5 = AVAILOFF5E - AVALLOFF5S +1;

clear a;

else

if $\mathrm{z} 10==$ RANGE5\%checks to see whether range of available offspring is equal to the required number of offspring

pos1 = AVAILOFF5S; pos2 = AVAILOFF5S + RANGE5 $-1 ; a=$ pos $1 ;$

while a $<$ pos2

Five $(a, 4)=$ Five $($ AVAILPAR5 + c31, 1$)$;

Five $(a+1,4)=$ Five $($ AVAlLPAR5 $+\mathrm{c} 31,1)$; Five $($ AVAILPAR5 $+\mathrm{c} 31,3)=2$;

$\mathrm{c} 31=\mathrm{c} 31+1 ; \mathrm{a}=\mathrm{a}+2 ;$

end

off $=$ pos2 +1;

while Five (off, 4) $\sim=0$

off $=$ off +1

end

AVAILOFF5S = Off; AVAILOFF5E = AVAILPAR5 + c31-1;

AVAILPAR5 = AVAILPAR5 + c31- $1 ; c 31=1 ;$ clear $a ;$

RANGE5 = AVAILOFF5E - AVALLOFF5S +1;

else

if $z 10>$ RANGE5\%checks to see whether range of available offspring is less than the required number of offspring

c $31=1$; if rem(RANGE 5,2$)==0$

pos1 = AVAILOFF5S; pos 2 = AVAILOFF5S + RANGE5 - $1 ; \mathrm{a}=$ pos $1 ;$ 
while $a<$ pos 2

Five $(a, 4)=$ Five $($ AVAILPAR5 $+\mathrm{c} 31,1)$;

Five $(a+1,4)=$ Five (AVATIPAR5 $+c 31,1) ;$ Five (AVAILPAR5 $+c 31,3)=2$

$\mathrm{c} 31=\mathrm{c} 31+1 ; \mathrm{a}=\mathrm{a}+2$;

end

AVAILOFF5S $=$ AVAILPAR5 + (z10/2) +1;

AVAILOFF5E = LAST_ROW_OF_MATRIX_FIVE

num = z10 - RANGE5; clear a

pos $1=$ AVAILOFF 5 S $;$ pOS 2 = AVAILOFF 5 S + num $-1 ; a=$ pos $1 ;$

while a pos2

Five $(a, 4)=$ Five (AVAILPAR5 + c31, 1);

Five $(a+1,4)=$ Five $($ AVAILPAR5 + c31,1); Five $($ AVAILPAR5 + c31, 3$)=2$

$\mathrm{c} 31=\mathrm{c} 31+1 ; \mathrm{a}=\mathrm{a}+2$;

else

pos $1=$ AVAILOFF5S; pos2 = AVALLOFF5S + RANGE5 $-1 ; \mathrm{C}=0$

for a $=$ pos $1:$ pos 2

$\mathrm{C}=\mathrm{C}+1$;

Five $(a, 4)=$ Five (AVAILPAR5 + c31, 1); Five (AVAILPAR5 + c31, 3) $=c$;

if Five(AVAILPAR5 + c31,3) == 2

$\mathrm{C} 31=\mathrm{C} 31+1 ; \mathrm{c}=0$;

end

AVAILOFF5S $=$ AVAILPAR5 $+(z 10 / 2)+1 ;$

AVAILOFF5E = LAST_ROW_OF_MATRIX_FIVE

num = z10 - RANGE5; clear a;

pos1 = AVALLOFF5s; pos2 = AVALLOFF5S + num -1 ;

for a $=$ pos $1:$ pos 2

$\mathrm{C}=\mathrm{C}+1$;

Five $(a, 4)=$ Five (AVAILPAR5 + c31, 1); Five (AVAILPAR5 + c31, 3) $=c$;

if Five(AVAILPAR5 + c31,3) ==

$C 31=C 31+1 ; C=0$;

end

end

end

end

en 
\% WRITE OUTPUT MATRICES IN SPREADSHEET

xlswrite('Thesis spreadsheet with m6 remodeled data (control order bounds).xlsm', One, 'Matlab output 1'), xlswrite('Thesis spreadsheet with m6 remodeled data (control order bounds).xlsm', Two, 'Matlab output 2');

xlswrite('Thesis spreadsheet with m6 remodeled data (control order bounds).xlsm', Three,'Matlab output 3'),

xlswrite('Thesis spreadsheet with m6 remodeled data (control order bounds).xlsm', Four, 'Matlab output 4'); xlswrite('Thesis spreadsheet with m6 remodeled data (control order bounds).xlsm', Five, 'Matlab output 5'); 
\% The user needs to specify percent distributions of segment diameters for various orders in

: Excel.The following code imposes diameter distributions of order 4 and order 5

․ vessel segments of one network onto another. The code reads the percent

\% distributions of various diameter values in the respective orders and imposes the diameter values

\% on the network being modified.

Four = xlsread('Orgthesisspreadsheet.xlsm', 'Order 4 DD'); \% reads the percent distribution of the

different diameters to be imposed on the order $4 \mathrm{~s}$

Five = xlsread('Orgthesisspreadsheet.xlsm', 'Order 5 DD'); $\%$ reads the percent distribution of the different diameters to be imposed on the order $5 \mathrm{~s}$

num_two $=14$; $\%$ enter \# of order $2 \mathrm{~s}$ in network undergoing change

num_three $=12$; $\%$ enter $\#$ of order $3 \mathrm{~s}$ in network undergoing change

num_five $=12$; $\%$ enter \# of order $5 \mathrm{~s}$ in the network undergoing change

NFour = xlsread('Thesis spreadsheet with ml remodeled data (COB) DD.xlsm', 'Label 4'); $\%$ reads the order 4 labels of the network being modified

NFive = xlsread('Thesis spreadsheet with m1 remodeled data (COB) DD.xlsm', 'Label 5'); $\%$ reads the order 5 labels of the network being modified

loop1 = length(Four) $-1 ;$ loop $2=$ length(Five) $-1 ; \mathrm{k}=1 ; \operatorname{maxval1}=$ length(NFour); $\operatorname{maxval2}=$ length(NFive) for $\mathrm{a}=1: 100 \mathrm{p} 1$

if $\operatorname{Four}(\mathrm{a}, 2) \sim=0$ 의 the percent distribution of a certain diameter value is not equal to 0 , then the code proceeds to assign the diameter value to segments of the network being modified

num $(1)=$ num four $* \operatorname{Four}(a, 2) ; \operatorname{num}=\operatorname{round}($ num $) ;$

if num $\sim=0$

$\operatorname{val}=\operatorname{Four}(\mathrm{a}, 1) ;$ inloop $=\mathrm{k}+$ num;

if inloop $<=$ maxvall

for $\mathrm{b}=\mathrm{k}$ : inloop

$\operatorname{NFour}(b, 2)=\operatorname{val}$

end

$\mathrm{k}=$ inloop +1 ;

end

end

end

clear a;

last $1=$ length(Four); lastloop = length (NFour);

$\operatorname{val}=$ Four $($ last 1,1$)$;

if $\operatorname{val} \sim=0$

for $a=1$ : lastloop

if $\operatorname{NFour}(a, 2)==0$

$\operatorname{NFour}(a, 2)=\operatorname{val}$

end 


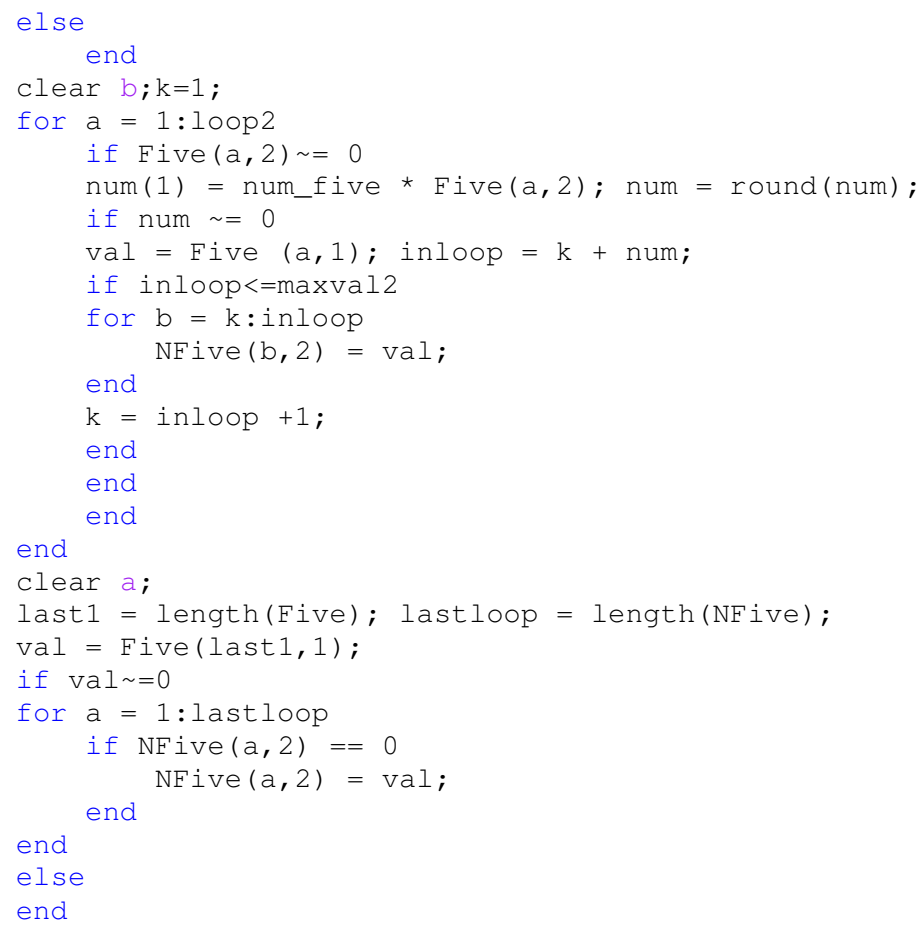

xlswrite('Thesis spreadsheet with m1 remodeled data (COB) DD.xlsm', NFour, 'Label 4'); xlswrite('Thesis spreadsheet with m1 remodeled data (COB) DD.xlsm', NFive, 'Label 5'), 
$\therefore$ The user needs to specify percent distributions of segment lengths for various orders in :Excel. The code then reads the percentage of segments with certain length

ovalues and the same percentage of segments of the respective order are

oassigned the length values in the network being changed. (Since there is a

ogreat variation in segment lengths even within a certain order, the

oaverage lengths for certain length ranges were determined and the percent

odistributions were accordingly calculated. The network being altered was

othen assigned the average length values based on the percent

odistribution.) The following code imposes length distributions of orders 2, 3 and 5

$\therefore$ vessel segments of one network onto another.

Two = xlsread('Thesis spreadsheet with m6 control data.xlsm', 'Order 2 LD');

Three $=$ xlsread('Thesis spreadsheet with m6 control data.xlsm', 'Order 3 LD');

Five = xlsread('Thesis spreadsheet with m6 control data.xlsm', 'Order 5 LD');

num_two $=79$; onter \# of order $2 \mathrm{~s}$ in network undergoing change

num three $=36$; $\frac{\circ}{0}$ enter \# of order $3 \mathrm{~s}$ in network undergoing change

num five = 14; \% enter \# of order $5 \mathrm{~s}$ in the network undergoing change

NTwO = xlsread('Thesis spreadsheet with m6 remodeled data (COB) LD.xlsm', 'Label 2');

NThree $=$ xlsread('Thesis spreadsheet with m6 remodeled data (COB) LD.xlsm', 'Label 3');

NFive $=$ xlsread('Thesis spreadsheet with m6 remodeled data (COB) LD.xlsm', 'Label 5-2')

loop1 = length(Two) $-1 ;$ loop 2 = length(Three) $-1 ;$ loop3 = length(Five); $\mathrm{k=1} ;$ maxval1 = length(NTwo) maxval2 = length (NThree) $;$ maxval3 = length (NFive)

for $a=1: 100 p$

if $\operatorname{Two}(a, 2) \sim=0$

num $(1)=$ num_two * Two $(a, 2) ; \operatorname{num}=\operatorname{round}($ num $)$;

if num $\sim=0$

val $=\operatorname{Two}(a, 1) ;$ inloop $=k+$ num

if inloop $<=$ maxvall

for $\mathrm{b}=\mathrm{k}$ : inloop

$\operatorname{NTwo}(b, 2)=\operatorname{val}$

end

$\mathrm{k}=$ inloop +1 ;

end

end

end

end

clear $a_{i}$

last $1=$ length (Two); lastloop = length (NTwo)

val $=$ Two $($ last 1,1$)$

if val $\sim=0$

for $\mathrm{a}=1$ : lastloop

if $\operatorname{NTwo}(a, 2)==0$ 
d

end

$\operatorname{NTwo}(a, 2)=\operatorname{val}$

else

end

clear $\mathrm{b} ; \mathrm{k}=1$;

for $a=1: 100 p 2$

if Three $(a, 2) \sim=0$

num $(1)=$ num_three $* \operatorname{Three}(a, 2) ; \operatorname{num}=$ round $($ num $)$;

if num $\sim=0$

val $=$ Three $(a, 1)$; inloop $=\mathrm{k}+$ num;

if inloop $<=$ maxval2

for $\mathrm{b}=\mathrm{k}$ : inloop

NThree $(b, 2)=$ val;

end

$\mathrm{k}=$ inloop +1 ;

end

end

end

$$
\text { end }
$$

clear a;

last $1=$ length (Three); lastloop = length (NThree);

val $=$ Three $($ last 1,1$)$;

if $\operatorname{val} \sim=0$

for $a=1$ : lastloop

if $\operatorname{NThree}(a, 2)==0$ NThree $(a, 2)=$ val;

end

else

lear $\mathrm{b} ; \mathrm{k}=1$;

for $a=1: 10003$

if $\operatorname{Five}(a, 2) \sim=0$

num $(1)=$ num_five $* \operatorname{Five}(a, 2) ;$ num $=$ round (num);

if num $\sim=0$

val $=$ Five $(a, 1) ;$ inloop $=\mathrm{k}+$ num;

if inloop $<=$ maxval3

for $\mathrm{b}=\mathrm{k}$ : inloop

$\operatorname{NFive}(b, 2)=\operatorname{val}$;

end 


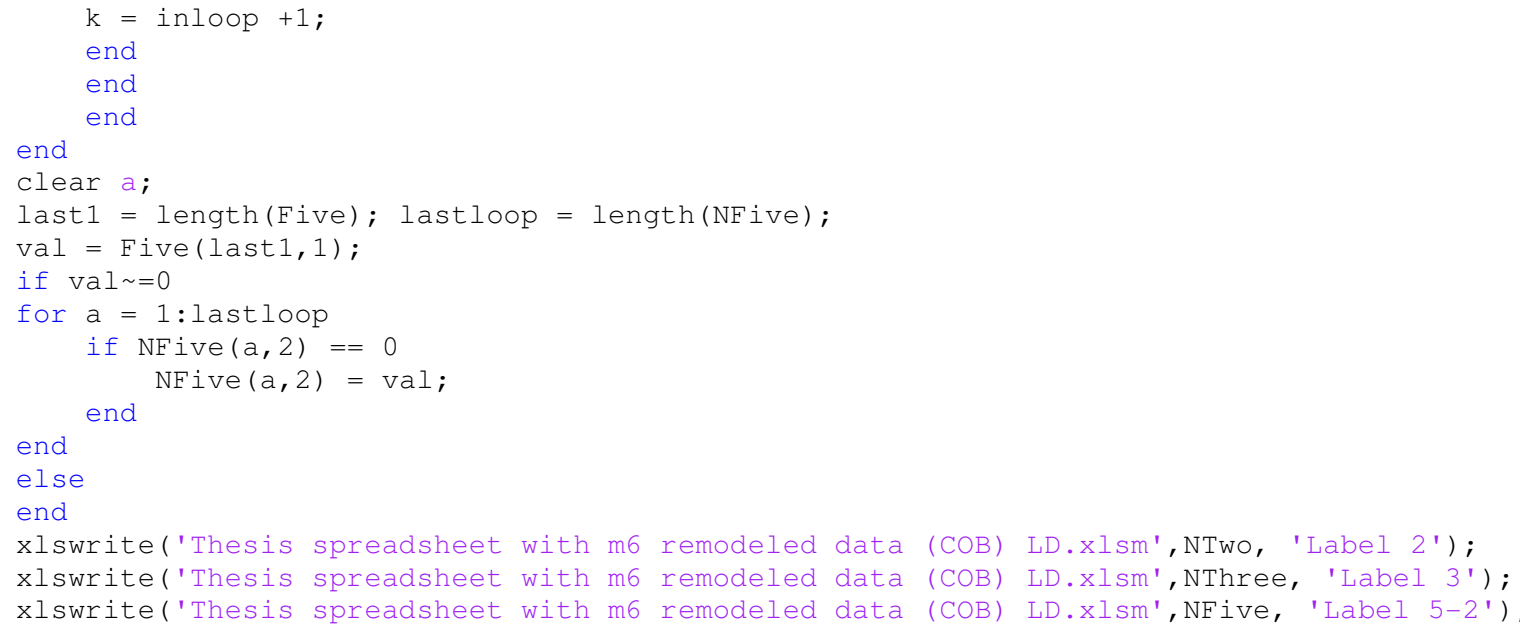

xlswrite('Thesis spreadsheet with m6 remodeled data (COB) LD.xlsm', NTwo, 'Label 2'); xlswrite('Thesis spreadsheet with m6 remodeled data (COB) LD.xlsm', NThree, 'Label 3'); xlswrite('Thesis spreadsheet with m6 remodeled data (COB) LD.xlsm', NFive, 'Label 5-2'); 
oSample Flow calculations in MATLAB

PMain $=10265.794$

$\mathrm{PC}=3999.66$

$\mathrm{CC}=1004.48909798338$

$\mathrm{C} 1=1060.28752058656$

$\mathrm{C} 2=1060.28752058656$;

$\mathrm{C} 3=1636.92794298374$;

$\mathrm{C} 4=1636.92794298374$;

C5 $=499.089171454082$;

$\mathrm{C} 6=499.089171454082$;

C $7=1309.542354387$;

$\mathrm{C} 8=4090.61543436171$

$\mathrm{C} 9=2683.85278648472$;

$\mathrm{C} 10=8417.07289534717$;

$\mathrm{C} 11=6524.84628053265$;

$\mathrm{C} 12=11224.6487518885$;

C13 $=32724.9234748937$;

$\mathrm{P} 7=\mathrm{PC}$;

$\mathrm{P} 6=\mathrm{PC}$

$\mathrm{P} 5=\mathrm{PC}$

$\mathrm{P} 4=\mathrm{PC} ;$

$\mathrm{P} 3=\mathrm{PC} ;$

$\mathrm{P} 2=\mathrm{PC} ;$

$\mathrm{P} 1=\mathrm{P} \mathrm{C}_{i}$

syms P8 P9 P10 P11 P12 P13 q8 q9 q10 q11 q12 q13

eq1 $=(\mathrm{PMain}-\mathrm{P} 13){ }^{\mathrm{C}} 13-((\mathrm{P} 13-\mathrm{P} 11) * \mathrm{C} 11+(\mathrm{P} 13-\mathrm{P} 12) * \mathrm{C} 12)$

eq2 $=(\mathrm{P} 13-\mathrm{P} 12){ }^{*} \mathrm{C} 12-\left((\mathrm{P} 12-\mathrm{P} 8){ }^{*} \mathrm{C} 8+(\mathrm{P} 12-\mathrm{P} 9){ }^{*} \mathrm{C} 9\right) ;$

eq $3=(\mathrm{P} 13-\mathrm{P} 11){ }^{*} \mathrm{C} 11-\left((\mathrm{P} 11-\mathrm{P} 7){ }^{*} \mathrm{C} 7+(\mathrm{P} 11-\mathrm{P} 10){ }^{*} \mathrm{C} 10\right) ;$

eq4 $=(\mathrm{P} 12-\mathrm{P} 9) * \mathrm{C} 9-((\mathrm{P} 9-\mathrm{P} 3) * \mathrm{C} 3+(\mathrm{P} 9-\mathrm{P} 4) * \mathrm{C} 4) ;$

eq5 $=(\mathrm{P} 12-\mathrm{P} 8){ }^{*} \mathrm{C} 8-\left((\mathrm{P} 8-\mathrm{P} 1){ }^{\circ} \mathrm{C} 1+(\mathrm{P} 8-\mathrm{P} 2){ }^{*} \mathrm{C} 2\right)$;

eq6 $=(\mathrm{P} 11-\mathrm{P} 10) * \mathrm{C} 10-\left((\mathrm{P} 10-\mathrm{P} 5) * \mathrm{C} 5+(\mathrm{P} 10-\mathrm{P} 6){ }^{*} \mathrm{C} 6\right)$

eq7 $=\mathrm{q} 9-(\mathrm{P} 12-\mathrm{P} 9) * \mathrm{C} 9$;

eq8 $=\mathrm{q} 8-(\mathrm{P} 12-\mathrm{P} 8) * \mathrm{C} 8$

eq9 $=$ q13 $-($ PMain $-\mathrm{P} 13){ }^{*} \mathrm{C} 13$

eq10 $=\mathrm{q} 12-(\mathrm{P} 13-\mathrm{P} 12) * \mathrm{C} 12$

eq11 $=\mathrm{q} 11-(\mathrm{P} 13-\mathrm{P} 11) * \mathrm{C} 11$;

eq12 $=$ q10 $-(\mathrm{P} 11-\mathrm{P} 10) * \mathrm{C} 10$;

vals = solve (eq1, eq2, eq3, eq4, eq5, eq6, eq7, eq8, eq9, eq10, eq11, eq12, P8, p9, P10, P11, P12, P13, q8, q9, q10, q11, q12, q13)

$\mathrm{P} 8 \mathrm{val}=\mathrm{vpa}(\mathrm{vals} . \mathrm{P} 8,7)$ 
P9val = vpa (vals.P9, 7)

P10val = vpa $($ vals.P10,7)

P11val = vpa (vals.P11,7)

$\mathrm{P} 12 \mathrm{val}=\mathrm{vpa}(\mathrm{vals} \cdot \mathrm{P} 12,7)$

P13val $=$ vpa(vals.P13,7

q8val $=\operatorname{vpa}(\operatorname{vals} . q 8,7)$

q9val = vpa (vals.q9,7)

q10val = vpa (vals.910,7)

q11val = vpa (vals.q11,7)

qlival -vpa(vals.q11,7)

q12val $=$ vpa $($ vals.q12, 7$)$

q13val $=\operatorname{vpa}(\mathrm{vals} \cdot q 13,7)$ 
Screenshot showing the organization of equations and constants in Excel that can be easily copied and pasted into Matlab to calculate flows and pressures

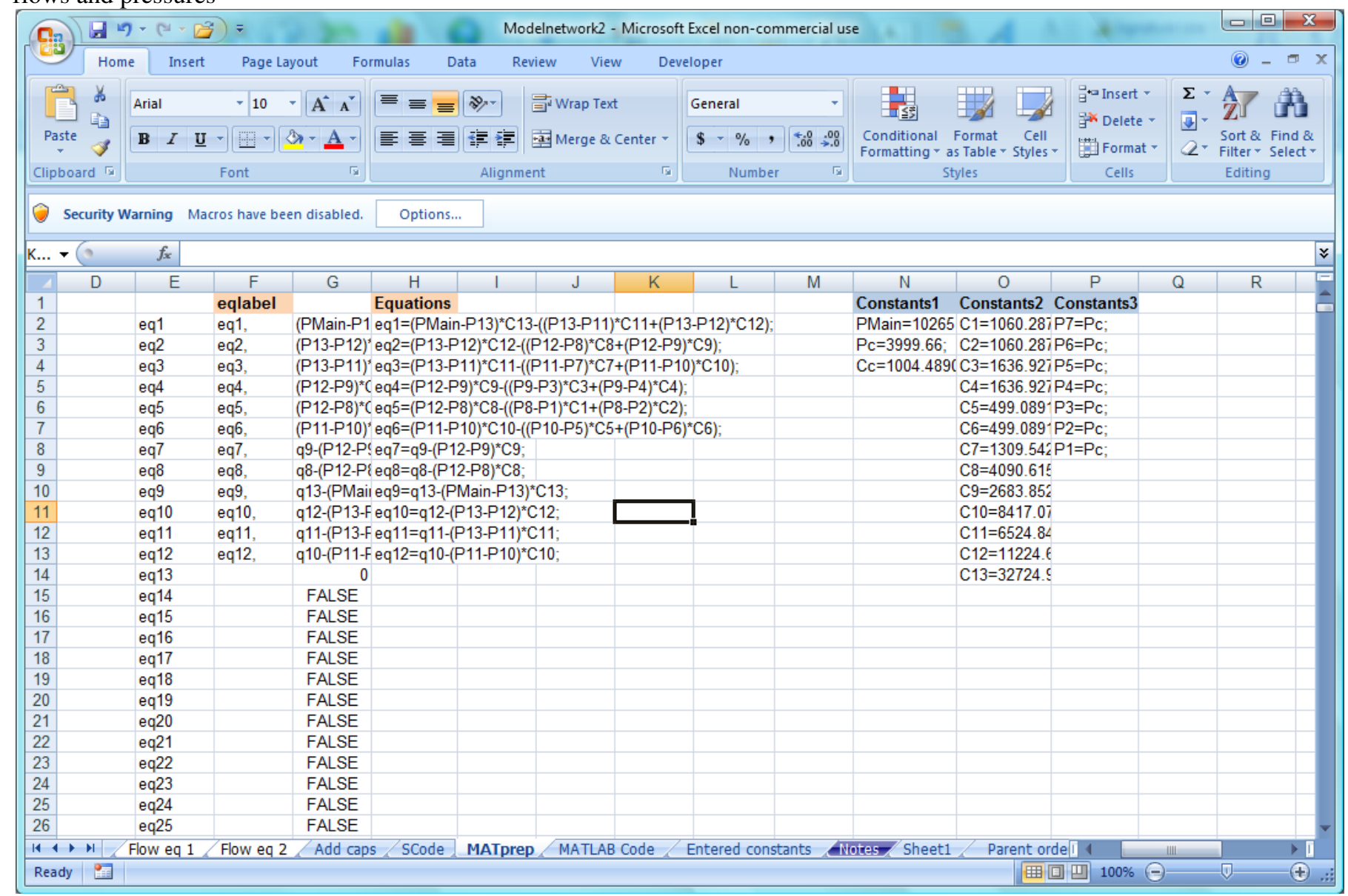


Mahfoudh, Samir (ELS-OXF) to me

show details Jun 10

Dear

We hereby grant you permission to reprint the material detailed below at no charge in your thesis subject to the following conditions: 1 . If any part of the material to be used (for example, figures) has appeared in our publication with credit or acknowledgement to another source, permission must also be sought from that source. If such permission is not obtained then that material may not be included in your publication/copies.2. Suitable acknowledgment to the source must be made, either as a footnote or in a reference list at the end of your publication, as follows: "This article was published in Publication title, Vol number, Author(s), Title of article, Page Nos, Copyright Elsevier (or appropriate Society name) (Year)."3. Your thesis may be submitted to your institution in either print or electronic form.4. Reproduction of this material is confined to the purpose for which permission is hereby given.5. This permission is granted for non-exclusive world English rights only. For other languages please reapply separately for each one required. Permission excludes use in an electronic form. Should you have a specific electronic project in mind please reapply for permission.6. This includes permission for UMI to supply single copies, on demand, of the complete thesis. Should your thesis be published commercially, please reapply for permission.Kind regards,

Sam Mahfoudh

Sam Mahfoudh :: Rights Assistant :: Elsevier

T: +44 (0)1865 843715 :: F: +44 (0)1865 853333

E: s.mahfoudh@elsevier.com-----Original Message-----

From: shilpi.ghosh@gmail.com [mailto:shilpi.ghosh@gmail.com]

Sent: 08 June 2010 01:27 
To: Health Permissions (ELS-PHI)

Subject: Obtain PermissionThis Email was sent from the Elsevier Corporate Web Site and is related to Obtain Permission form:

Product: Customer Support

Component: Obtain Permission

Web server: http://www.elsevier.com

IP address: 98.248 .111 .46

Client: $\quad$ Mozilla/4.0 (compatible; MSIE 8.0; Windows NT 6.0; Trident/4.0; GTB6.5; SLCC1; .NET CLR 2.0.50727; Media Center PC 5.0; .NET CLR 1.1.4322; .NET CLR 3.5.30729; .NET CLR 3.0.30729)

Invoked from:

http://www.elsevier.com/wps/find/obtainpermissionform.cws_home?isSubmitted=yes\&navigateXmlFileName=/store/scstargets/prd53/act/fra mework_support/obtainpermission.xml

Request From:

Shilpi Ghosh

Cal Poly, San Luis Obispo

38730 Lexington Street Apt. 154

94536

Fremont

United States

Contact Details: 
Telephone:

Fax:

Email Address: shilpi.ghosh@gmail.com

To use the following material:

ISSN/ISBN: $\quad 978-0721602400$

Title: $\quad$ Textbook of Medical Physiology Eleventh edition

Author(s): Guyton and Hall

Volume: $\quad$ N/A

Issue: $\quad \mathrm{N} / \mathrm{A}$

Year: $\quad 2006$

Pages: $\quad 162-162$

Article title: Overview of the Circulation

How much of the requested material is to be used:

Figure 14-1

Are you the author: No

Author at institute: No

How/where will the requested material be used: [how_used]

Details: 
I would like to use the figure in the "Introduction" chapter of my thesis. My thesis involves a study of the morphology and hemodynamics of normal and pathologically remodeled vascular networks.

Additional Info:

[acronym]

- end -

Elsevier Limited. Registered Office: The Boulevard, Langford Lane, Kidlington, Oxford, OX5 1GB, United Kingdom, Registration No. 1982084 (England and Wales). 